University of Tennessee Health Science Center

UTHSC Digital Commons

\title{
$5-2011$
}

\section{Insights into p53-Dependent Apoptotic Signaling and Cell Fate vis- a-vis Functional Cooperation among BCL-xL, Cytoplasmic p53, and PUMA}

John C. Fisher

University of Tennessee Health Science Center

Follow this and additional works at: https://dc.uthsc.edu/dissertations

Part of the Medical Cell Biology Commons, and the Neoplasms Commons

\section{Recommended Citation}

Fisher, John C. , "Insights into p53-Dependent Apoptotic Signaling and Cell Fate vis-a-vis Functional Cooperation among BCL-XL, Cytoplasmic p53, and PUMA" (2011). Theses and Dissertations (ETD). Paper 80. http://dx.doi.org/10.21007/etd.cghs.2011.0094. 


\title{
Insights into p53-Dependent Apoptotic Signaling and Cell Fate vis-a-vis Functional Cooperation among BCL-xL, Cytoplasmic p53, and PUMA
}

\author{
Abstract \\ Following DNA damage, nuclear p53 induces the expression of PUMA (p53 upregulated modulator of \\ apoptosis), a BH3-only protein that binds and inhibits the anti-apoptotic BCL-2 repertoire, including \\ BCL-xL. Structural investigations of PUMA and the BCL-XLXPUMA BH3 domain complex by X-ray \\ crystallography and nuclear magnetic resonance (NMR) spectroscopy reveal a novel, PUMA-induced, \\ domain-swapped dimerization of $B C L-x L$ that requires a $\pi$-stacking interaction between PUMA W71 and \\ $B C L-x L$ H113. PUMA is an intrinsically disordered protein, but upon interaction with BCL-XL, PUMA W71 \\ and the PUMA BH3 domain residues fold into an alpha helix and subtly remodel $B C L-x L$ to trigger its \\ dimerization. Wild type PUMA or a PUMA mutant incapable of promoting BCL-xL dimerization (PUMA \\ W71A) equivalently inhibit the anti-apoptotic BCL-2 repertoire to sensitize for death receptor-activated \\ apoptosis, but only wild type PUMA promotes p53-dependent DNA damage-induced apoptosis. \\ Biochemical and cellular data demonstrate that PUMA-mediated structural remodeling and dimerization \\ of $B C L-x L$ modulates its affinity for cytosolic $p 53$, providing a detailed mechanism of $B C L-x L$, cytosolic \\ p53, and PUMA functional cooperation. Our data suggest that within the BCL-2 family, ligand \\ binding-induced, domain-swapped dimerization is a critical control point to increase signal transduction \\ complexity within the apoptotic pathways.

\section{Document Type \\ Dissertation} \\ Degree Name \\ Doctor of Philosophy $(\mathrm{PhD})$ \\ Program \\ Biomedical Sciences \\ Research Advisor \\ Richard W. Kriwacki, Ph.D. \\ Keywords \\ Apoptosis, BCL-xL, domain swap, p53, PUMA \\ Subject Categories \\ Diseases | Medical Cell Biology | Medical Sciences | Medicine and Health Sciences | Neoplasms
}


Insights into p53-Dependent Apoptotic Signaling and Cell Fate vis-a-vis Functional

Cooperation among BCL-xL, Cytoplasmic p53, and PUMA

\author{
A Dissertation \\ Presented for \\ The Graduate Studies Council \\ The University of Tennessee \\ Health Science Center
}

\author{
In Partial Fulfillment \\ Of the Requirements for the Degree \\ Doctor of Philosophy \\ From The University of Tennessee
}

By

John C. Fisher

May 2011 
Copyright (C) 2011 by John C. Fisher.

All rights reserved. 


\section{DEDICATION}

This dissertation is dedicated to my mother, Vicki R. Fisher, whose loving heart, indissoluble strength, inspiring spirit, and beautiful smile will forever be my inspiration. 


\section{ACKNOWLEDGEMENTS}

I would like to acknowledge all those who supported and assisted me during my research. First and foremost, I would to thank Dr. Richard Kriwacki for his support and guidance as well as for giving me the opportunities and freedoms to develop and pursue my project within his laboratory. My gratitude is also extended to Cheon Gil Park for his ability to keep me grounded and focused on life's big picture. I would also like to thank every member of the Kriwacki laboratory, the Department of Structural Biology at St. Jude Children's Research Hospital, and the faculty and administration within the Integrated Program for Biomedical Sciences and the College of Medicine at The University of Tennessee Health Science Center. I would also like to thank my family for the unwavering support they have constantly provided me throughout my long journey through graduate school.

My thanks are also given to Dr. Jerry Chipuk in the laboratory of Dr. Douglas Green (Chair of Immunology, St. Jude Children's Research Hospital) for performing a series of truly elegant experiments throughout our collaboration. Without his support, the amazing success of this project would not have been possible. Additionally, I would like to thank Dr. Douglas Green for not only serving as a member of my committee, but also for constantly providing key insights into the project as well as reagents and laboratory space for me to continue performing in vivo experiments fundamental to my project's success.

I would also like to thank the other members of my committee: Drs. Suzanne Baker, Brenda Schulman, and Stephen White for their assistance and time over the past six years. I am incredibly lucky to have had their advices at such an early stage of my career.

Further, I would like to thank Dr. Amanda Nourse (Hartwell Center, St. Jude Children's Research Hospital) for performing numerous protein interaction studies. In addition, I would like to thank Dr. Darcie Miller and Ms. Mi-Kyung Yun in the Department of Structural Biology at St. Jude Children's Research Hospital for their help with data collection and structural refinement, respectively.

Finally, I would like to acknowledge the National Institutes of Health (NIH), the Alma and Hal Reagan Fellowship awarded to me from The University of Tennessee Health Science Center, and the American Lebanese and Syrian Associated Charities (ALSAC) for providing the funding for my research project. 


\begin{abstract}
Following DNA damage, nuclear p53 induces the expression of PUMA (p53 upregulated modulator of apoptosis), a BH3-only protein that binds and inhibits the anti-apoptotic BCL-2 repertoire, including BCL-xL. Structural investigations of PUMA and the BCL-xL.PUMA BH3 domain complex by X-ray crystallography and nuclear magnetic resonance (NMR) spectroscopy reveal a novel, PUMA-induced, domain-swapped dimerization of BCL-xL that requires a $\pi$-stacking interaction between PUMA W71 and BCL-xL H113. PUMA is an intrinsically disordered protein, but upon interaction with BCL-xL, PUMA W71 and the PUMA BH3 domain residues fold into an alpha helix and subtly remodel BCL-xL to trigger its dimerization. Wild type PUMA or a PUMA mutant incapable of promoting BCL-xL dimerization (PUMA W71A) equivalently inhibit the anti-apoptotic BCL-2 repertoire to sensitize for death receptor-activated apoptosis, but only wild type PUMA promotes p53-dependent, DNA damage-induced apoptosis. Biochemical and cellular data demonstrate that PUMA-mediated structural remodeling and dimerization of BCL-xL modulates its affinity for cytosolic p53, providing a detailed mechanism of BCL-xL, cytosolic p53, and PUMA functional cooperation. Our data suggest that within the BCL-2 family, ligand binding-induced, domain-swapped dimerization is a critical control point to increase signal transduction complexity within the apoptotic pathways.
\end{abstract}




\section{TABLE OF CONTENTS}

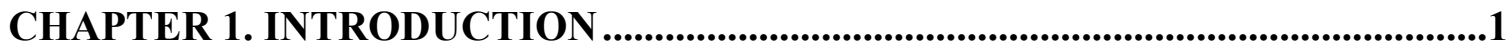

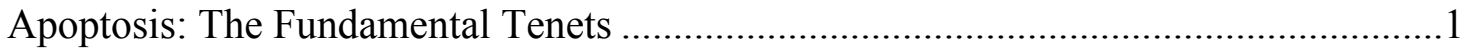

The BCL-2 Family Proteins...................................................................................4

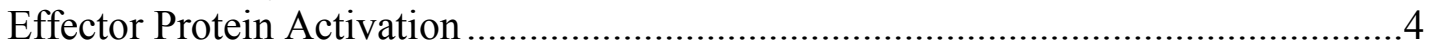

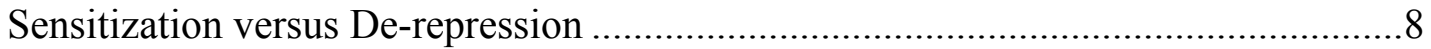

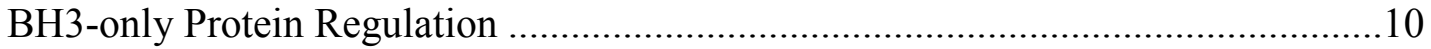

Structural Biology of the BCL-2 Family Proteins ....................................................11

The p53 Tumor Suppressor and Apoptosis................................................................ 15

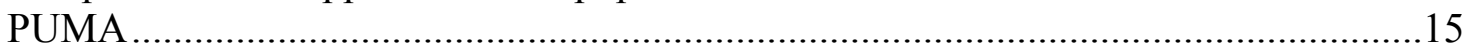

Puma Knockout Mice Are Resistant to Apoptosis .................................................17

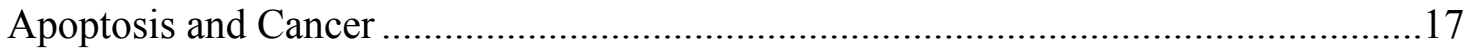

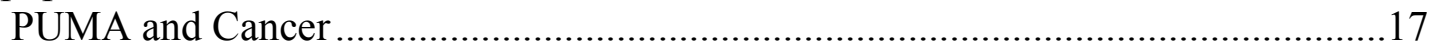

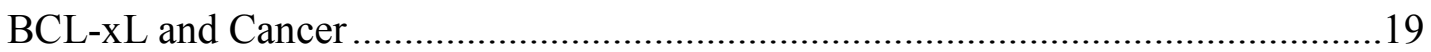

Cytoplasmic p53 and Its Transcription-Independent, Pro-apoptotic Function..............20

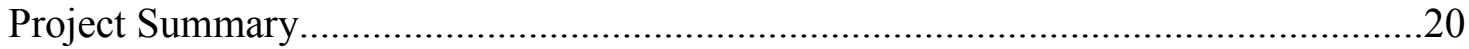

CHAPTER 2. REAGENTS AND EXPERIMENTAL PROCEDURES ...................22

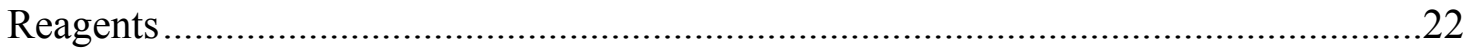

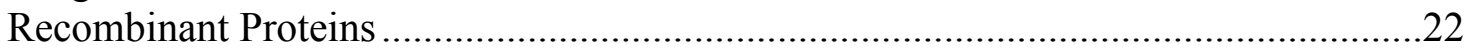

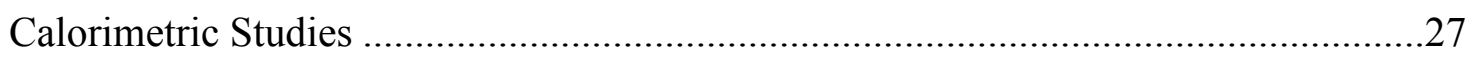

Circular Dichroism Spectropolarimetry..................................................................28

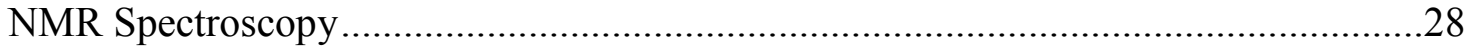

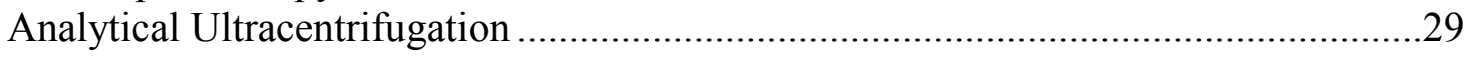

Crystallization, Data Collection, and Refinement .............................................. 30

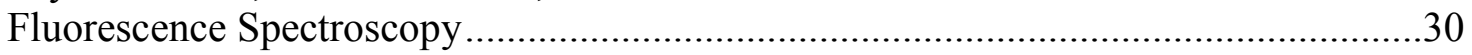

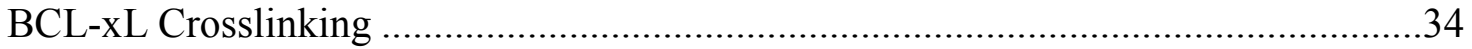

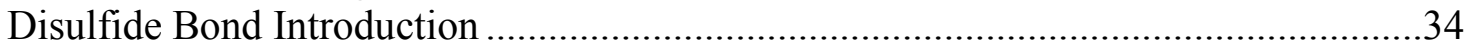

Electrophoresis, Immunoblotting, and Co-immunoprecipitation ................................34

Heavy Membrane Preparation and Cytochrome c Release ........................................35

Cell Culture, Transfection, and Survival Assays ........................................................36

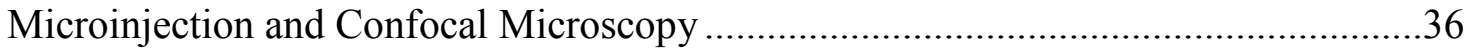

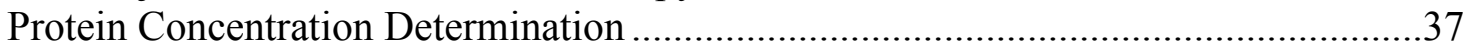

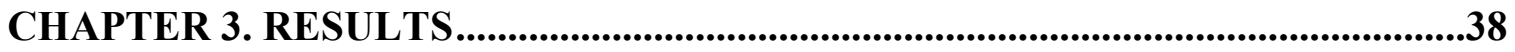

PUMA Is an Intrinsically Disordered Protein that Induces BCL-xL Dimerization .......38

PUMA-induced BCL-xL Dimer Is Not in Dynamic Equilibrium ..............................42

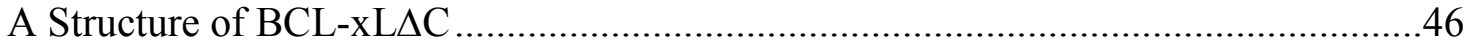

The Structure of the BCL-xL $\Delta \mathrm{C} \cdot \mathrm{PUMA}{ }^{\mathrm{BH} 3}$ Complex ..............................................46

Analyses of the BCL-xL $\Delta C \cdot \mathrm{PUMA}^{\mathrm{BH} 3}$ Interaction Reveal the Mechanism for

PUMA-induced BCL-xL Dimerization of BCL-xL ..............................................50

PUMA W71 Is Required to Promote p53-dependent MOMP and Apoptosis ..............59

Dimeric BCL-xL $\Delta \mathrm{C}$ Does Not Inhibit p53-dependent MOMP .................................69 
CHAPTER 4. DISCUSSION .............................................................................................77

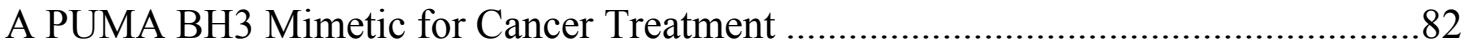

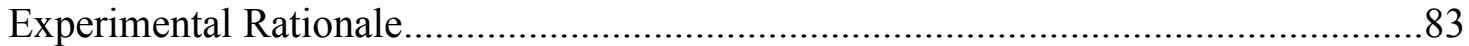

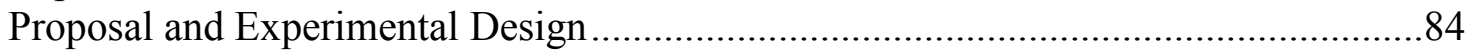

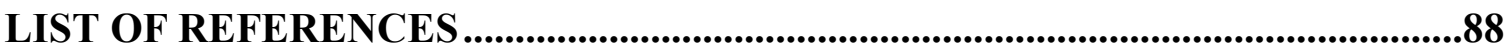

APPENDIX A. SUPPLIMENTARY TABLES AND FIGURES..................................101

APPENDIX B. RECOMBINANT PROTEIN SEQUENCES .........................................108

APPENDIX C. BUFFERS...............................................................................................111

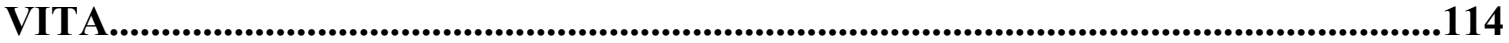




\section{LIST OF TABLES}

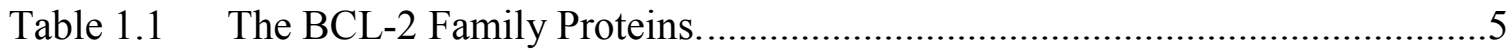

Table 2.1 BCL-xL $\Delta$ C Structure Data and Refinement Statistics................................32

Table 2.2 BCL-xL $\Delta C \cdot$ PUMA $^{\mathrm{BH} 3}$ Structure Data and Refinement Statistics...............33

Table 3.1 BH3 Domain Peptide Sequences. ..........................................................44

Table A.1 Oligonucleotide Primers for Site-directed Mutagenesis ........................... 102

Table A.2 Details of Recombinant Protein Expression Vectors...............................103

Table A.3 Example of Raw Fluorescence Data Used to Solve for $K_{\mathrm{d}}$ by Non-linear Regression .................................................................................... 105

Table A.4 Thermodynamic Parameters for BCL-xL Binding to Various BH3 Domain Peptides ........................................................................... 107 


\section{LIST OF FIGURES}

Figure 1.1 Intrinsic and Extrinsic Apoptotic Pathways.................................................

Figure 1.2 BCL-2 Family Domain Organization. ……………………………..........6

Figure 1.3 Proposed Model of BAX Activation..............................................................

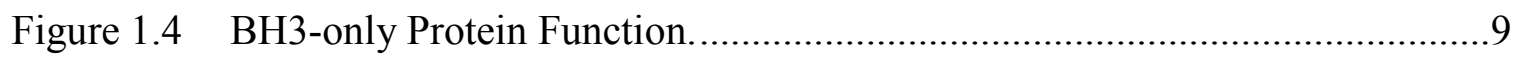

Figure 1.5 Structures of the BCL-2 Family Protein Core. ……………………............12

Figure 1.6 Sequence Alignment of BH3 Domains from BH3-only Proteins.................13

Figure 1.7 Structural Comparison of Apo Anti-apoptotic Proteins Compared with the Respective BH3 Domain-bound Forms. .................................................14

Figure 1.8 p53 Regulation. ................................................................................16

Figure 1.9 Comparison of PUMA Alpha and Beta Isoforms........................................18

Figure 2.1 Recombinant PUMA and BCL-xL Proteins Are Active...............................23

Figure 2.2 SDS-PAGE Analysis of the Indicated Recombinant Proteins......................24

Figure 2.3 Protein Crystals and Respective Diffraction Data Images.............................31

Figure 3.1 PUMA Is an Intrinsically Disordered Protein..................................................39

Figure 3.2 The BH3 Domain of PUMA Is Sufficient for Binding BCL-xL..................40

Figure 3.3 Analytical Ultracentrifugation of Indicated BCL-xL $\Delta C$ Species..................41

Figure 3.4 Native PAGE Analysis of PUMA-Anti-apoptotic BCL-2 Family Protein

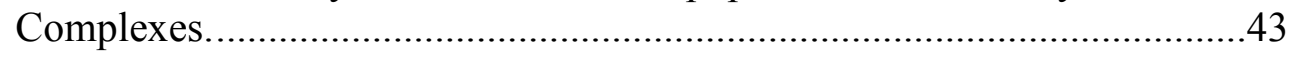

Figure 3.5 ITC Analysis of BH3 Domain Peptides Binding BCL-xLAC ......................45

Figure 3.6 The PUMA-induced BCL-xL Dimer Shows No Signs of Dynamic Equilibrium.

Figure 3.7 Structures of BCL-xL $\Delta \mathrm{C}$ and the BCL-xL $\Delta \mathrm{C} \cdot \mathrm{PUMA}^{\mathrm{BH} 3}$ Complex. ...........49

Figure 3.8 The BCL-xL $\Delta C \cdot P U M A{ }^{\mathrm{BH} 3}$ Complex Is Domain Swapped in Solution.......51

Figure 3.9 Structure-dependent Crosslinking of the PUMA-induced BCL-xL Domain Swapped Dimer Reveals that PUMA-induced BCL-xL Dimerization Occurs via Domain Swapping. 


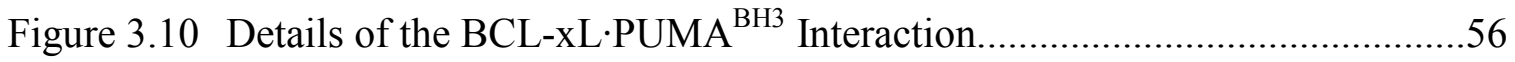

Figure 3.11 Verification of PUMA ${ }^{\mathrm{BH} 3}$ Side Chain Interactions with BCL-xL $\Delta \mathrm{C}$ by Fluorescence Anisotropy.........................................................................57

Figure 3.12 PUMA W71 Forms a $\pi$-stacking Interaction with BCL-xL H113................58

Figure 3.13 PUMA-induced Dimerization of BCL-xL is Mediated Uniquely by the $\pi$-stacking Interaction between PUMA W71 and BCL-xL H113.................60

Figure 3.14 The W71A Point Mutation in PUMA ${ }^{\mathrm{BH} 3}$ Does Not Affect Binding to Other Anti-apoptotic BCL-2 Family Proteins .62

Figure 3.15 PUMA W71 Specifically Promotes DNA Damage-induced Apoptosis.......63

Figure 3.16 P53 and PUMA Bind Different Solvent-exposed Surfaces on BCL-xL.......65

Figure 3.17 PUMA Induces BCL-xL Dimerization in Cells............................................66

Figure 3.18 C8-BID and p53 Exhibit Direct Activation of BAX and Induction of MOMP, whereas PUMA Does Not.

Figure 3.19 PUMA and PUMA ${ }^{\mathrm{BH} 3}$ Uniquely Release $\mathrm{p} 53^{\mathrm{UVIP}}$ from BCL-xL $\Delta \mathrm{C}$...........68

Figure 3.20 PUMA W71 Is Critical for De-repression of p53 but Not C8-BID from BCL-xL. . .70

Figure 3.21 PUMA-Induced BCL-xL $\Delta$ C Dimerization Correlates with p53 ${ }^{\text {UVIP }}$ dependent BAX Activation and Subsequent Cytochrome c Release .71

Figure 3.22 Only Monomeric BCL-xL $\Delta \mathrm{C}$ Binds and Inhibits p53. .72

Figure 3.23 BCL-xL Monomer and BCL-xL $\Delta$ C Apo-dimer Differentially Regulate $\mathrm{p} 53^{\mathrm{UVIP}}$ and C8-BID-induced Cytochrome c Release.

Figure 3.24 BCL-xL Monomer and BCL-xL $\Delta$ C Apo-dimer Differentially Regulate TNF and UV-induced MOMP.

Figure 4.1 PUMA-induced Dimerization of BCL-xL Releases p53 via Burial of the p53 Binding Epitope. .78

Figure 4.2 Structural Comparison of BCL-xL $\Delta \mathrm{C} \cdot \mathrm{PUMA}^{\mathrm{BH} 3}, \mathrm{MCL}^{-1} \Delta \mathrm{C} \cdot \mathrm{PUMA}^{\mathrm{BH} 3}$, and BFL- $1 \Delta \mathrm{C} \cdot \mathrm{PUMA}^{\mathrm{BH} 3}$ Complexes.

Figure 4.3 Target Areas of BCL-xL Used to Screen for a Novel Small Molecule PUMA $^{\mathrm{BH} 3}$ Mimetic .86

Figure A.1 Reversed Phase C4 HPLC Chromatogram of PUMA. 104 
Figure A.2 Determining the $K_{\mathrm{d}}$ and $-\Delta \Delta \mathrm{G}$ Value for Fluorescein-labeled Wild Type PUMA $^{\mathrm{BH} 3}$ 


\section{LIST OF ABBREVIATIONS}

\begin{tabular}{ll}
$\alpha$ & Alpha \\
$\Delta$ & Delta \\
$\mu 1$ & Microliter \\
$\mu \mathrm{M}$ & Micromolar \\
1D & One-dimensional \\
2D & Two-dimensional \\
APAF1 & Apoptotic protease activating factor 1 \\
BAD & BCL-2 antagonist of cell death \\
BAK & BCL-2 antagonist/killer \\
BAX & BCL-2-associated x protein \\
BC & BCL-2 core \\
BCL-2 & B cell lymphoma 2 \\
BCL-xL & BCL-2-related gene, long isoform \\
BFL-1 & BCL-2-related gene A1 \\
BH & BCL-2 homology \\
BID & BH3-interacting-domain death agonist \\
BIK & BCL-2-interacting killer \\
BIM & BCL-2-interacting mediator of cell death \\
BMH & Bismaleimidohexane \\
C8-BID & Caspase-8 cleaved BID \\
CD & Circular diochroism \\
CDK & Cyclin-dependent kinase \\
Cip & Cyclin-dependent kinase inhibitory protein \\
C-terminal & Carboxyl-terminal \\
DMEM & Dulbecco's modified eagle medium \\
DTT & Dithiothreitol \\
E.coli & Escherichia coli \\
EDTA & Ethylene diaminetetraacetic acid \\
EGTA & Ethylene glycol bis (2-amino-ethylether)-N, N, N', N'-tetraacetic \\
& acid \\
FADD & FAS-associated death domain \\
FBS & Fetal bovine serum \\
FRET & Förster resonance energy transfer \\
GFP & Green fluorescent protein \\
GST & Glutathione s-transferase \\
HPLC & High performance liquid chromatography \\
HRK & Harakiri \\
HSQC & Heteronuclear single quantum coherence \\
IDP & Intrinsically disordered protein \\
IPTG & Isopropyl $\beta$-D-1-thiogalactopyranoside \\
I/R & Infarction/Reperfusion \\
ITC & Isothermal titration calorimetry \\
K & Degrees Kelvin \\
& \\
\hline
\end{tabular}




\begin{tabular}{|c|c|}
\hline kcal & Kilocalorie \\
\hline $\mathrm{KCl}$ & Potassium chloride \\
\hline $\mathrm{kDa}$ & Kilodalton \\
\hline KID & Kinase inhibitory domain \\
\hline KIP & Kinase inhibitory protein \\
\hline LB & Luria-Bertani media \\
\hline LH & Linker helix \\
\hline LUVs & Large unilamellar vesicles \\
\hline MCL-1 & Myeloid cell leukemia 1 \\
\hline $\mathrm{MgCl}_{2}$ & Magnesium chloride \\
\hline $\mathrm{ml}$ & Milliliter \\
\hline MOMP & Mitochondrial outer membrane permeabilization \\
\hline $\mathrm{NaCl}$ & Sodium chloride \\
\hline ng & Nanogram \\
\hline $\mathrm{nm}$ & Nanometer \\
\hline $\mathrm{nM}$ & Nanomolar \\
\hline NMR & Nuclear magnetic resonance \\
\hline N-terminal & Amino-terminal \\
\hline OMM & Outer mitochondrial membrane \\
\hline PBS & Phosphate buffered saline \\
\hline PUMA & P53-upregulated modulator of apoptosis \\
\hline SAHB & Stabilized $\alpha$ helix of BCL-2 domain peptide \\
\hline SAR & Structure activity relationships \\
\hline SD200 & Superdex 200 \\
\hline SDS-PAGE & Sodium dodecyl sulfate polyacrylamide gel electrophoresis \\
\hline TAD & Transactivation domain \\
\hline TFA & Trifluoroacetic acid \\
\hline TM & Transmembrane \\
\hline TCEP & Tris [2-carboxyethyl] phosphine \\
\hline $\mathrm{TNF}$ & Tumor Necrosis Factor \\
\hline TRADD & TNFR1-associated death domain \\
\hline TROSY & Transverse relaxation optimized spectroscopy \\
\hline UV & Ultraviolet \\
\hline
\end{tabular}




\section{CHAPTER 1. INTRODUCTION}

\section{Apoptosis: The Fundamental Tenets}

Apoptosis is a genetically controlled process through which multicellular organisms dismantle damaged or superfluous cells (Hengartner et al., 1992). Apoptosis has roles in tissue development (Braithwaite et al., 2005), homeostasis, and disease (Danial and Korsmeyer, 2004). It is defined by a sequence of molecular and morphological changes that result in the labeling, packaging, and elimination of dying cells. Cells committed to die via apoptosis following developmental cues, stress, or infection are culled by phagocytes to prevent a host inflammatory response. "Scrambling" of the plasma membrane occurs resulting in the exposure of phosphatidyl serine, which is usually restricted to the cytoplasmic face of the cell membrane, to the extracellular environment (Bevers and Williamson, 2010). In vivo, the externalized phosphatidyl serine and other induced signals allow recognition by phagocytic cells, which engulf apoptotic cells (Fadok et al., 2000; Hanayama et al., 2002; Savill and Fadok, 2000). There are two general signaling pathways that trigger apoptosis, the extrinsic and mitochondrial apoptotic pathways (Figure 1.1) (Tait and Green, 2010). How death signals are transduced to activate caspases (cysteinyl aspartate proteases that efficiently coordinate cellular dismantling) differentiate the two pathways. Deathreceptor ligation by death ligands is responsible for the induction of the extrinsic pathway (Muppidi et al., 2004). The mitochondrial pathway is activated by various developmental cues or cytotoxic insults.

Death-receptor ligation by death ligands is responsible for the induction of the extrinsic pathway (Muppidi et al., 2004) (Figure 1.1). For example, tumor necrosis factor (TNF) binds to its death receptor, TNFR, which causes the recruitment of adaptor molecules the adaptor molecule FAS-associated death domain (FADD). Recruitment of caspase -8 by TRADD results in dimerization, interdomain cleavage, and finally activation of caspase- 8 (Boatright et al., 2003; Oberst et al., 2010). Caspase- 8 activation subsequently induces activation of downstream effector caspases, caspase-3, -6 or -7 . In some cell types, the extrinsic pathway collaborates with the mitochondrial pathway via caspase-8 cleavage-mediated activation of protein $\mathrm{BH} 3$-interacting-domain death agonist (BID), a pro-apoptotic BH3-only protein (Kaufmann et al., 2007; Yin et al., 1999). Caspase- 8 activated BID (C8-BID) is subsequently able to engage the mitochondrial apoptotic pathway (Figure 1.1).

The mitochondrial pathway is responsible for most apoptotic cell death in vertebrates and is engaged by cellular stresses such as DNA damage, cell cycle defects, extracellular matrix detachment, hypoxia, or loss of cell survival factors (Vousden and Prives, 2009). This pathway is regulated by complex interactions among the BCL-2 family proteins (Figure 1.1), which regulate the integrity of the outer mitochondrial membrane (OMM) (Green and Evan, 2002). The pivotal event that commits a cell to die via the intrinsic apoptotic pathway is mitochondrial outer membrane permeabilization (MOMP) (Kluck et al., 1997; Yang et al., 1997). As a result of MOMP, cytochrome c 
Figure 1.1 Intrinsic and Extrinsic Apoptotic Pathways.

(A) Intrinsic apoptotic stimuli, such as DNA damage activate B cell lymphoma 2 (BCL2) homology 3 (BH3)-only proteins leading to BCL-2-associated $X$ protein (BAX) and BCL-2 antagonist or killer (BAK) activation and mitochondrial outer membrane permeabilization (MOMP). Anti-apoptotic BCL-2 proteins prevent MOMP by binding $\mathrm{BH} 3-$ only proteins and activated BAX or BAK. Following MOMP, release of various proteins promotes caspase activation and apoptosis. Cytochrome $\mathrm{c}$ binds apoptotic protease-activating factor 1 (APAF1), inducing its oligomerization and thereby forming the apoptosome that recruits and activates initiator caspase 9. Caspase 9 cleaves and activates executioner caspases, caspase 3 and caspase 7, leading to apoptosis. (B) The extrinsic apoptotic pathway is initiated by ligation of death receptors with their cognate ligands, leading to the recruitment of adaptor molecules such as FAS-associated death domain protein (FADD) and then caspase 8 . This results in the dimerization and activation of caspase- 8 , which directly cleaves and activates caspases -3 and -7 , leading to apoptosis. Crosstalk between the extrinsic and intrinsic pathways occurs through caspase 8 cleavage and activation of the $\mathrm{BH} 3$-only protein $\mathrm{BH} 3$-interacting domain death agonist (BID). Reprinted with permission by Macmillan Publishers Ltd: Tait, S.W., and Green, D.R. (2010). Mitochondria and cell death: outer membrane permeabilization and beyond. Nat Rev Mol Cell Biol 11, 621-632. 


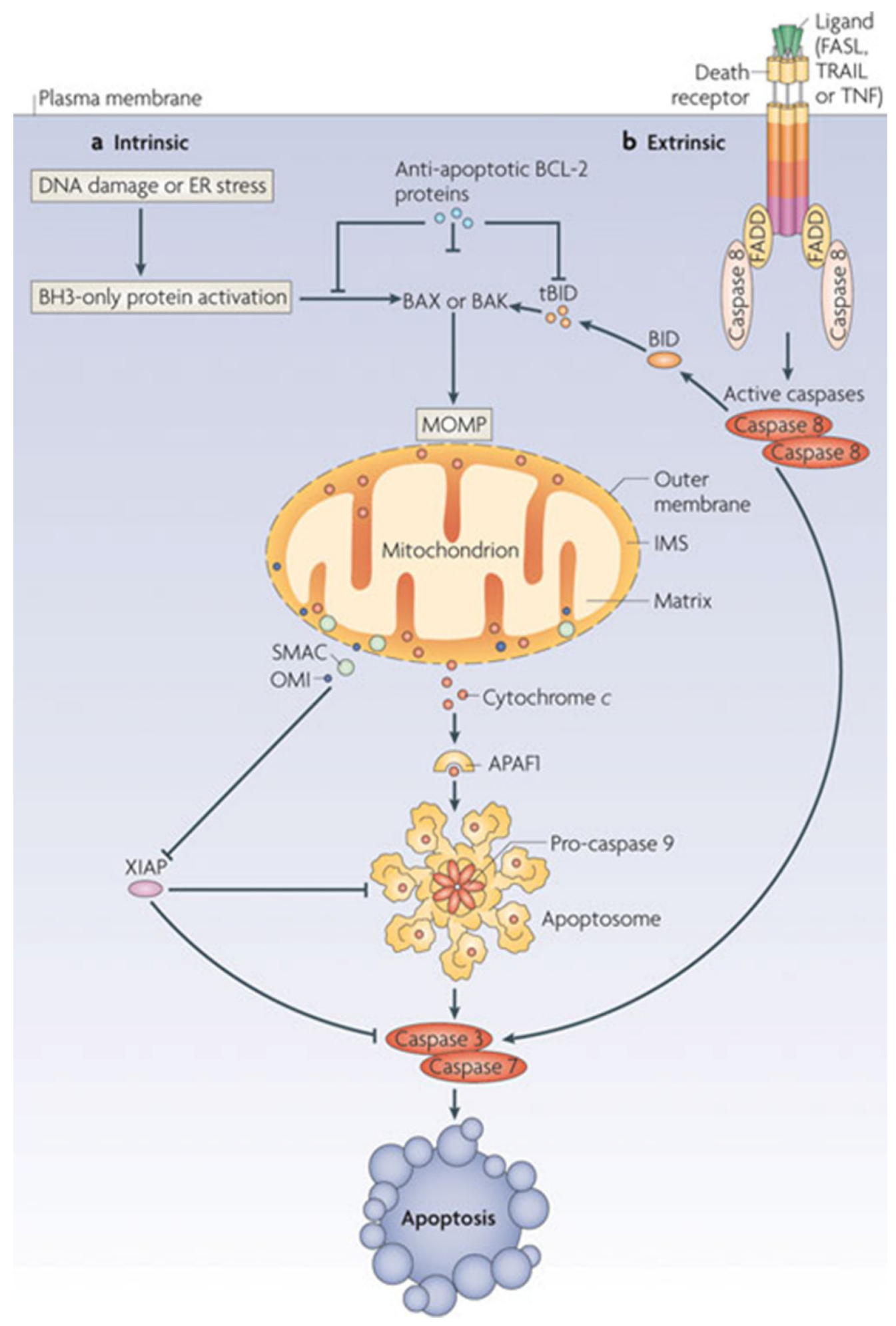


is released from the mitochondrial intermembrane space and induces apoptotic protease activating factor 1 (APAF1) oligomerization into a caspase activating platform called the apoptosome (Zou et al., 1997). The apoptosome binds and promotes the dimerization and activation of initiator caspase-9 (Hao et al., 2005; Pop et al., 2006; Riedl and Salvesen, 2007), which subsequently activates the executioner caspases-3 and -7 (Li et al., 1997; Salvesen and Dixit, 1997; Zou et al., 1997). These executioner caspases are responsible for the phenotypic hallmarks of apoptosis, such as chromatin condensation, plasma membrane asymmetry, and cellular blebbing (Kerr et al., 1972; Salvesen and Dixit, 1997).

\section{The BCL-2 Family Proteins}

The B-cell lymphoma 2 (BCL-2) protein and its relatives are functionally classified as either anti-apoptotic or pro-apoptotic. Most cells express an assortment of both anti-apoptotic and pro-apoptotic BCL-2 proteins (Table 1.1), and the regulation of their interactions dictates cell survival or commitment to apoptosis (Chipuk and Green, 2008).

Anti-apoptotic BCL-2 proteins contain four BCL-2 homology (BH) domains (BH1-4) (Figure 1.2). The anti-apoptotic repertoire is comprised of BCL-2-related gene A1 (A1), BCL-2, BCL-2-related gene, long isoform (BCL-xL), BCL-w, and myeloid cell leukemia 1 (MCL-1) (Youle and Strasser, 2008). These proteins preserve the integrity of the outer mitochondrial membrane (OMM) by directly inhibiting pro-apoptotic BCL-2 proteins.

The pro-apoptotic BCL-2 members are divided into 2 groups: the multidomain effector proteins and the BH3-only proteins (Youle and Strasser, 2008). The multidomain effector proteins, BCL-2 antagonist/killer (BAK) and BCL-2-associated X protein (BAX), were originally described as being composed of only three $\mathrm{BH}$ domains (BH1-3). Structure-based alignment of folded BCL-2 family proteins redefined the BH4 domain motif and revealed that it is structurally conserved within the N-terminal helix of both multidomain effector and anti-apoptotic BCL-2 proteins (Kvansakul et al., 2008) (Figure 1.2). As their name implies, the $\mathrm{BH} 3$-only proteins contain only the $\mathrm{BH} 3$ domain (Figure 1.2) (Youle and Strasser, 2008).

\section{Effector Protein Activation}

BAK is constitutively anchored to the OMM by its carboxyl-terminal (C-terminal) transmembrane helix, whereas BAX exists as an inactive monomer within the cytosol (Wei et al., 2000). Following BH3-only protein-induced activation, BAK and BAX form homo-oligomers of various sizes which insert into the OMM and promote MOMP and ultimately apoptotic cell death (Figure 1.3). BAK and BAX are essential for MOMP and induction of the mitochondrial apoptotic pathway, as $\mathrm{Bak}^{-/-} \mathrm{Bax}^{-/-}$cells are refractory to apoptosis induced by an assortment of death stimuli (Wei et al., 2001). 
Table 1.1 The BCL-2 Family Proteins.

\begin{tabular}{ccc}
\hline $\begin{array}{c}\text { Anti-apoptotic } \\
\text { Proteins }\end{array}$ & $\begin{array}{c}\text { Pro-apoptotic } \\
\text { Effector Proteins }\end{array}$ & $\begin{array}{c}\text { Pro-apoptotic } \\
\text { BH3-only Proteins }\end{array}$ \\
\hline A1 & BAK & Direct Activators \\
BCL-2 & BAX & BID \\
BCL-w & BIM \\
BCL-xL & De-repressor/ \\
& Sensitizers \\
MCL-1 & BAD \\
& BMF \\
& HRK \\
& Noxa \\
& & PUMA \\
\hline
\end{tabular}




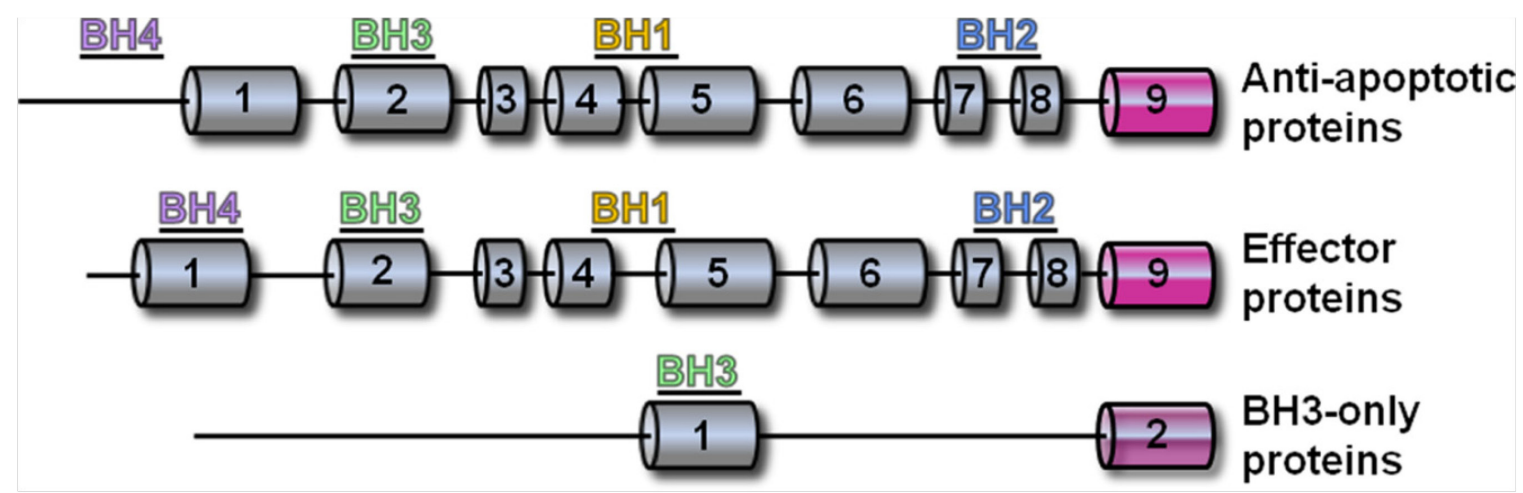

Figure 1.2 BCL-2 Family Domain Organization.

Schematic representations of the domain arrangements of the indicated BCL-2 family member groups. Grey cylinders represent alpha $(\alpha)$ helices and magenta cylinders represent helical transmembrane domains (TM). The BCL-2 homology $(\mathrm{BH})$ domains are indicated above each schematic: $\mathrm{BH} 4, \mathrm{BH} 3, \mathrm{BH} 1$, and $\mathrm{BH} 2$ domains are pink, green, yellow, and blue, respectively. 


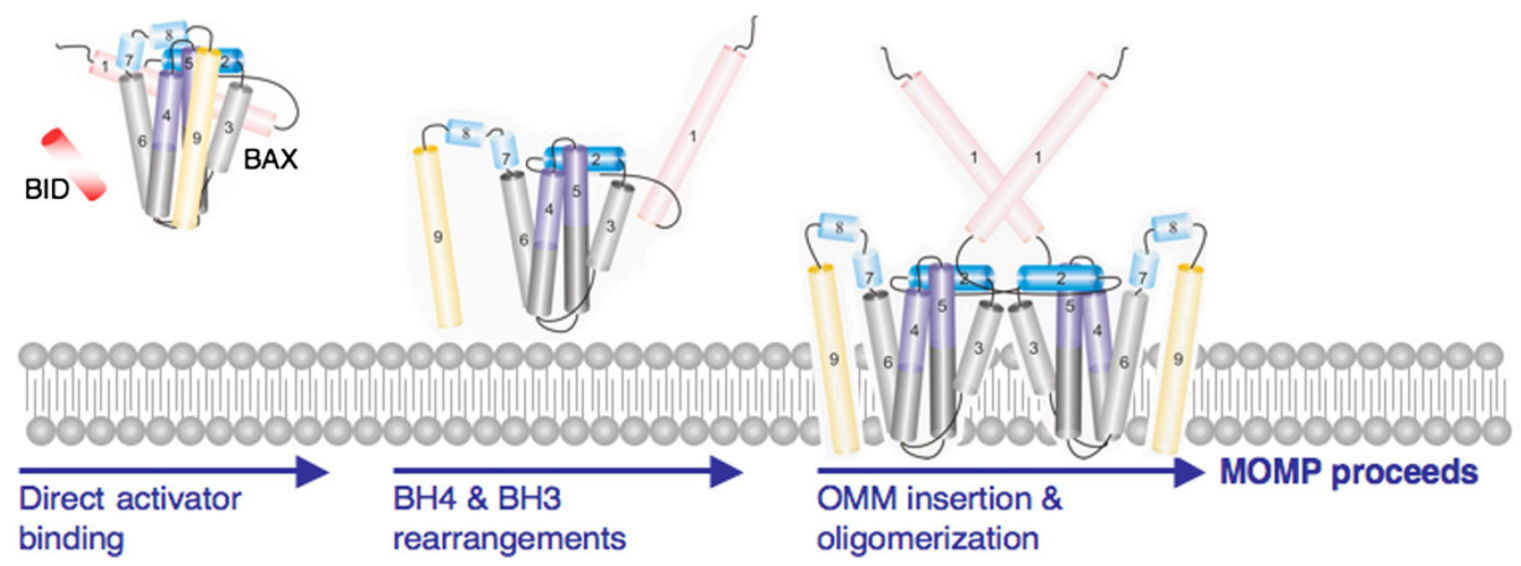

Figure 1.3 Proposed Model of BAX Activation.

Soluble BAX interacts with a direct activator and the OMM to promote stable N-terminal exposure, and BAX $\alpha 5, \alpha 6$, and $\alpha 9$ insert within the OMM. Reprinted with permission by Macmillan Publishers Ltd: Chipuk, J.E., Moldoveanu, T., Llambi, F., Parsons, M.J., and Green, D.R. (2010). The BCL-2 family reunion. Mol Cell 37, 299-310. 
Each BH3-only protein has a unique binding profile for the anti-apoptotic repertoire (Figure 1.4A). There are two different types of BH3-only proteins. One subset interacts only with the anti-apoptotic BCL-2 repertoire and is referred to as "sensitizer"' and/or "de-repressor' BH3-only proteins. The other subset, "direct activator" BH3-only proteins, can directly interact with both the anti-apoptotic BCL-2 repertoire and the proapoptotic effector proteins (Chipuk et al., 2010). The currently known direct activator $\mathrm{BH} 3$-only proteins include BID and BCL-2-interacting mediator of cell death (BIM). These BH3-only proteins directly induce BAK and/or BAX oligomerization and MOMP (Chipuk et al., 2010).

\section{Sensitization versus De-repression}

As discussed above, BID and BIM directly induce effector protein activation and function (Kuwana et al., 2002; Wei et al., 2000). The presence of the BH3 domain of $\mathrm{BH} 3$-only proteins is necessary and sufficient for $\mathrm{BH} 3$-only protein function. For example, BH3 domain peptides derived from BID and BIM induce BAK and BAX oligomerization and pore-forming activity with isolated mitochondria or large unilamellar vesicles (LUVs, lipid vesicles that faithfully mimic the OMM) (Kuwana et al., 2005; Letai et al., 2002).

BH3-only proteins, such as BAD, BCL-2-interacting killer (BIK), Harakiri (HRK), Noxa, and PUMA function not by directly activating BAK or BAX but rather mediate BAK and/or BAX activation indirectly by binding the anti-apoptotic repertoire (Chen et al., 2005; Chipuk et al., 2008; Kuwana et al., 2005; Letai et al., 2002). The terms "'sensitizer', and "de-repressor' are used to indicate the outcomes of interaction between $\mathrm{BH} 3$-only proteins and anti-apoptotic BCL-2 proteins. These $\mathrm{BH} 3$-only proteins establish two distinct mechanisms to indirectly promote effector protein activation: sensitization and de-repression (Chipuk et al., 2008; Kuwana et al., 2005; Letai et al., 2002) (Figure 1.4B-C).

Sensitization lowers the pro-apoptotic signaling threshold for BAK and/or BAX activation and MOMP but does not directly induce apoptosis (Figure 1.4B). In this scenario, anti-apoptotic BCL-2 proteins become saturated with sensitizer BH3-only proteins (via formation of anti-apoptotic protein sensitizer $\mathrm{BH} 3$-only protein complexes), effectively preventing inhibition of subsequent direct activator $\mathrm{BH} 3$-only protein signaling and function (Chipuk et al., 2010). For example, if BCL-2 is associated with PUMA (which potently inhibits the entire BCL-2 repertoire, any future induction of BIM would not be inhibited and MOMP would proceed. In the absence of PUMA, BIM would be sequestered and the cell may survive.

In the case of de-repression, a direct activator $\mathrm{BH} 3$-only protein is bound by an anti-apoptotic BCL-2 protein, and a subsequent BH3-only protein releases the direct activator to promote MOMP (Figure 1.4C). For example, reparable cellular stress can induce BIM function, but this activity would be blocked by the anti-apoptotic repertoire and the cell would survive. If however, a de-repressor $\mathrm{BH} 3$-only protein is induces while 

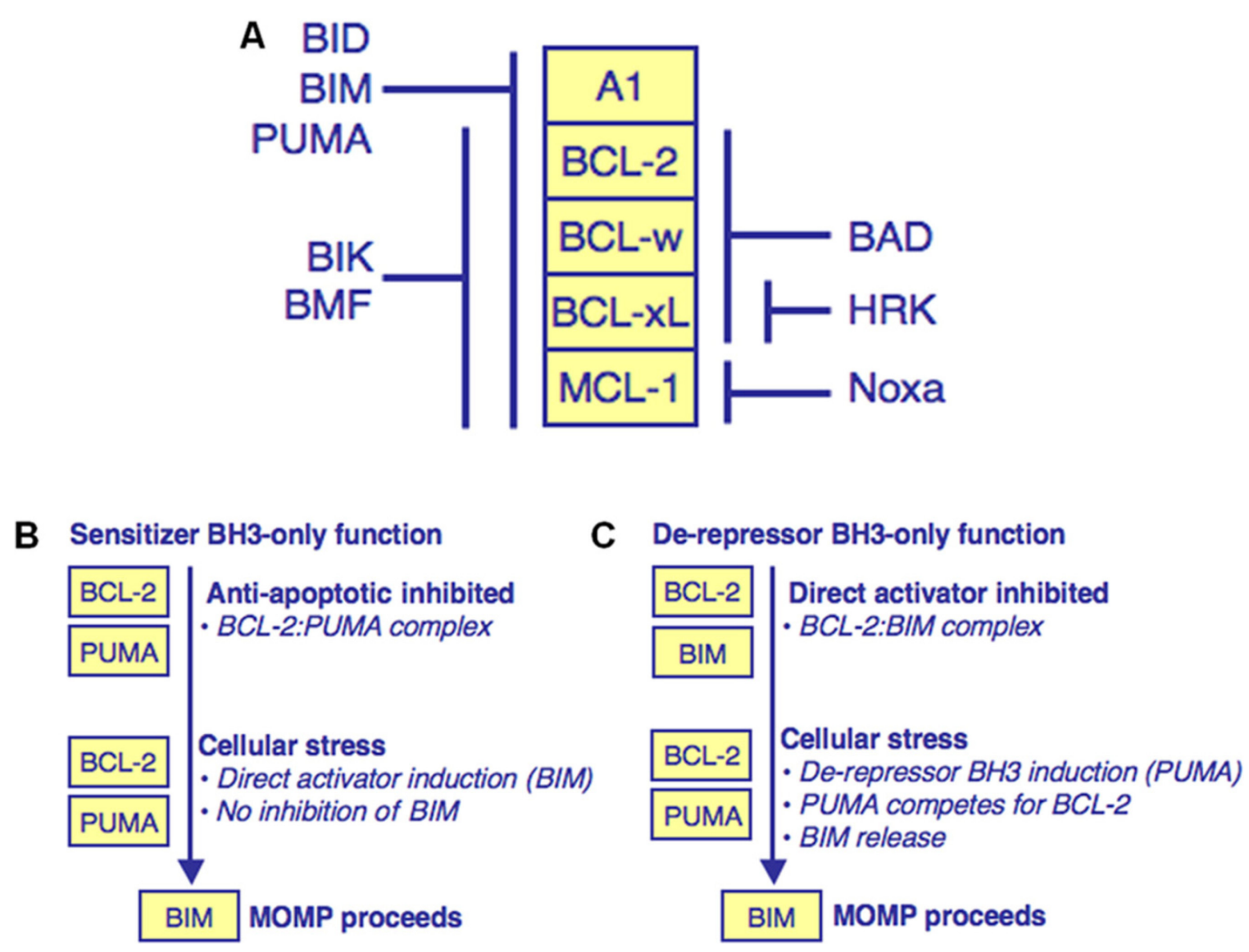

\section{Figure 1.4 BH3-only Protein Function.}

(A) The anti-apoptotic BCL-2 protein binding profiles for the BH3-only proteins. Antiapoptotic proteins are shown within yellow boxes. Solid blue lines indicate inhibitory interactions. (B) Sensitizer BH3-only protein function. A sensitizer BH3-only protein inhibits the anti-apoptotic BCL-2 repertoire. Following minimal cellular stress, a direct activator is induced but cannot be inhibited and MOMP proceeds. (C) De-repressor BH3only protein function. A direct activator is sequestered by an anti-apoptotic BCL-2 protein. Following cellular stress, a de-repressor BH3-only protein is induced and competes with the direct activator for binding to the anti-apoptotic repertoire. When the direct activator is released, MOMP proceeds. Reprinted with permission by Macmillan Publishers Ltd: Chipuk, J.E., Moldoveanu, T., Llambi, F., Parsons, M.J., and Green, D.R. (2010). The BCL-2 family reunion. Mol Cell 37, 299-310. 
the direct activator is sequestered, the latter can be released, allowing for MOMP (Chipuk et al., 2010). De-repressor BH3-only proteins release direct activator BH3-only proteins via direct competition for the same binding epitope on the various anti-apoptotic proteins present within the cell. Thus whether a direct activator BH3-only protein is de-repressed depends on what anti-apoptotic proteins are present, the binding affinities for both the direct activator and the de-repressor $\mathrm{BH} 3$-only protein for these particular anti-apoptotic proteins, as well as the relative concentration of the induced/activated de-repressor $\mathrm{BH} 3-$ only protein (Certo et al., 2006; Letai et al., 2002).

Studies using Förster resonance energy transfer (FRET) demonstrated that derepression and consequential direct activation occur via protein'protein interactions that are not readily detected in the absence of membranes (Lovell et al., 2008). For example, activated BID (C8-BID) was bound by BCL-xL, and this interaction could be disrupted by BAD. C8-BID subsequently interacted directly with BAX and induced subsequent BAX oligomerization and LUV permeabilization. In sum, MOMP readily occurs following the combined efforts of direct activator and sensitizer/de-repressor BH3-only proteins both in vitro (Chipuk et al., 2008) and in vivo (Merino et al., 2009).

\section{BH3-only Protein Regulation}

The multiplicity and intricate regulation of mammalian $\mathrm{BH} 3$-only proteins combine to allow these pro-apoptotic proteins to provide exquisite control over apoptosis. As such, activated $\mathrm{BH} 3$-only proteins act as death signal mediators following a multitude of distinct cellular stress scenarios (Chipuk et al., 2010). Transcriptional upregulation is one means by which BH3-only protein activation can occur. Noxa and PUMA are induced by the tumor suppressor p53 in response to DNA damage (Nakano and Vousden, 2001; Oda et al., 2000; Yu et al., 2001), and BIM is induced by the class O forkhead box transcription factor-3A (FOXO3A) in response to growth-factor deprivation (Dijkers et al., 2000) as well as by the CEBP homologous protein (CHOP) transcription factor in response to endoplasmic reticulum stress (Puthalakath et al., 2007). Post-translational modification is another means for mediating BH3-only protein activation. BAD activation ensues following growth-factor deprivation-induced dephosphorylation (Zha et al., 1996). As described above, BID is activated by caspase-8-mediated proteolytic cleavage (Li et al., 1998; Luo et al., 1998). BIM activation follows its release from the dynein motor complex (Puthalakath et al., 1999) or by loss of extracellular signalregulated kinase (ERK)-mediated phosphorylation (which targets BIM for ubiquitinmediated proteasomal degradation in healthy cells) (Akiyama et al., 2003; Ley et al., 2005). BMF is activated by release from actin-myosin motor complexes (Puthalakath et al., 2001), and BIK is activated by an unknown mechanism in response to inhibition of protein synthesis (Shimazu et al., 2007). 


\section{Structural Biology of the BCL-2 Family Proteins}

The BCL-2 family can be divided into two groups based on protein structure: folded, globular proteins and intrinsically disordered proteins (IDPs) (Hinds et al., 2007). The primary amino acid sequence of IDPs are rich in polar and charged amino acids, while the number of hydrophobic amino acids is low enough to limit the presence of a hydrophobic core (Dyson and Wright, 2005). In isolation, IDPs lack secondary and tertiary structure (Wang et al., 2011). Rather they exist as a multitude of conformational ensembles (Mittag and Forman-Kay, 2007). All multidomain anti-apoptotic and proapoptotic effector proteins are folded, helical bundle proteins that share a conserved BCL-2 core (BC) (Figure 1.5) (Petros et al., 2004b).

With the exception of its conserved $\mathrm{BH} 3$ domain, the $\mathrm{BH} 3$-only protein BID exhibits the lowest degree of primary sequence conservation among the folded BCL-2 family members. Nonetheless, the solution structure of BID revealed that the $\mathrm{BC}$ is in fact structurally conserved (Chou et al., 1999). Other BH3-only proteins are intrinsically disordered (Hinds et al., 2007), and the BH3 domains fold into $\alpha$ helices upon binding to hydrophobic grooves of BCL-2 family proteins.

The BC was first observed in the structure of BCL-xL (Muchmore et al., 1996a) and is an approximately $20 \mathrm{kDa}$ globular domain comprised of six amphipathic $\alpha$ helices arranged around a pair of central, buried helices. A hydrophobic groove formed by the BH1-3 domains is found on one side of the BC. The C-terminal $\alpha$ helix of BAX, BCL-w, and probably full-length $\mathrm{BCL}-\mathrm{xL}$ resides within this hydrophobic groove, whereas the groove is solvent exposed in other folded BCL-2 family members that are constitutively tethered to the OMM (Figure 1.5). This hydrophobic groove is requisite for heterodimerization between the $\mathrm{BH} 3$ domains of pro-apoptotic $\mathrm{BH} 3$-only proteins and the anti-apoptotic BCL-2 repertoire. The BH4 region is structurally defined by the conserved $\alpha 1$ helix and stabilizes the $\mathrm{BC}$ hydrophobic groove by packing against the remaining three $\mathrm{BH}$ domains (Figure 1.5).

Examination of the various $\mathrm{BC}$ binding grooves reveals a hydrophobic groove of variable width lining one side of the BC (Figure 1.5), decorated on either side by distinct combinations of aliphatic, aromatic, polar, and charged amino acids. In addition to the amino acid sequence of the various BH3-only domains (Figure 1.6), the degree of flexibility of the various anti-apoptotic proteins' $\mathrm{BC}$ grooves, the structural differences in the $\mathrm{BC}$ grooves and the amino acids surrounding them define anti-apoptotic protein selectivity for BH3-only proteins (Czabotar et al., 2007; Day et al., 2005; Day et al., 2008; Denisov et al., 2006; Feng et al., 2007; Herman et al., 2008; Petros et al., 2000; Sattler et al., 1997; Smits et al., 2008).

It is clear that the helical BCs of the anti-apoptotic proteins exhibit varying degrees of dynamics which dictate their structural transitions upon $\mathrm{BH} 3$ domain binding (Smits et al., 2008). Comparing the structures of apo BCL-xL and apo MCL-1 with the respective BIM-bound structures provides a clear example of the variable dynamics of the $\mathrm{BC}$ binding groove (Figure 1.7). The most striking structural difference between apo 

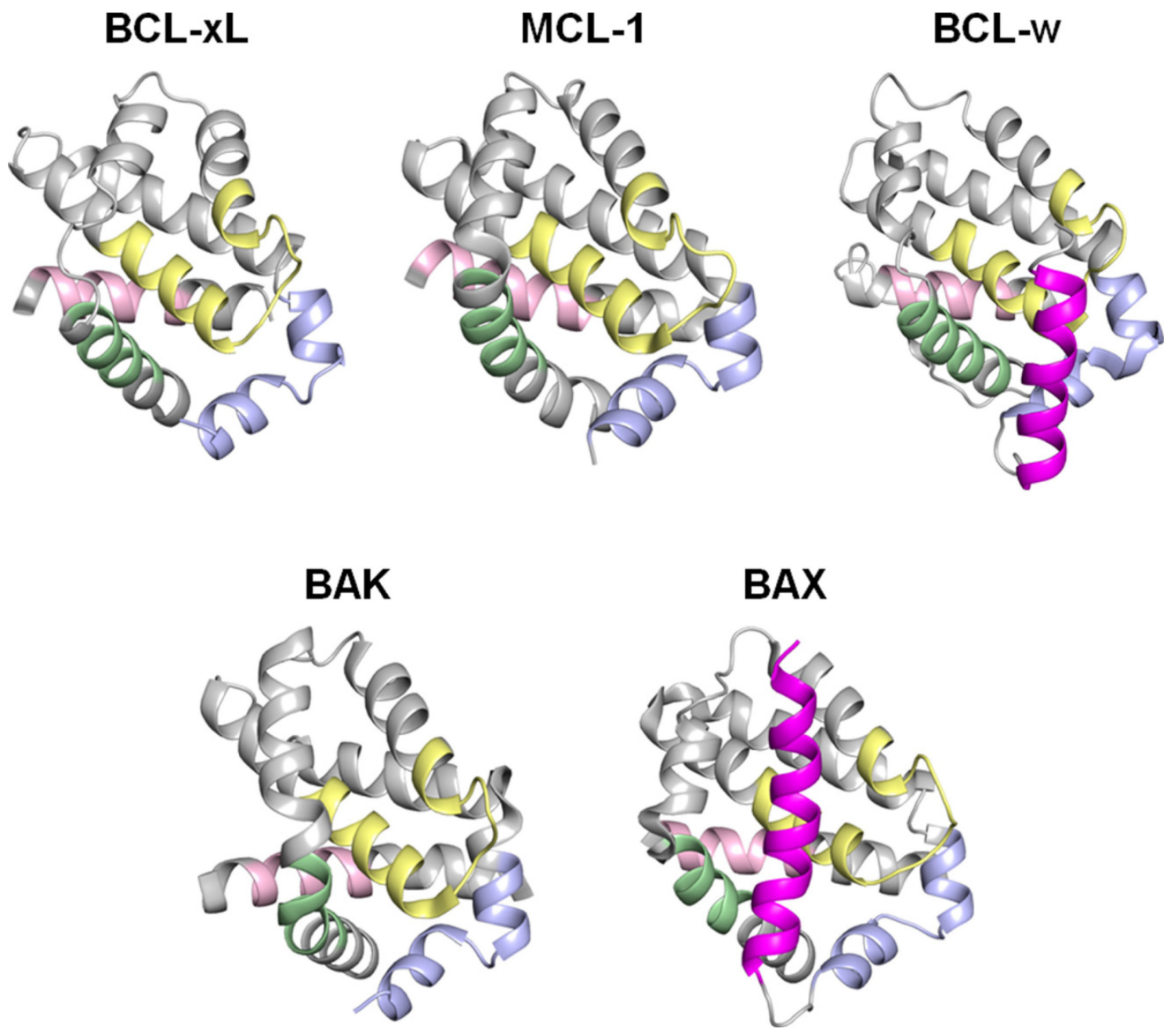

Figure 1.5 Structures of the BCL-2 Family Protein Core.

Ribbon representation of multidomain anti-apoptotic and pro-apoptotic BCL-2 family proteins: BCL-xL (PDB ID:1LXL), MCL-1 (PDB ID:1WSX), BCL-w (PDB ID:1MK3), BAK (PDB ID:2IMT), and BAX (PDB ID:1F16). The BH4, BH3, BH1, and BH2 domains are pink, green, yellow, and blue, respectively. Carboxyl terminal (C-terminal) transmembrane (TM) domains are magenta. 
BAD NLWAAQR YRELRR SDE VDSFKK BAK PSSTMGQVGRLAI IGDD INRRYDS BID QEDIIRNIARHLAQVGDSMDSIPP BIK CMEGSDATARLAC GDE DVSLRA BIM DMRPEIWIAQLRRIGDE NAYYAR BMF QHQAEVQ $\triangle A R K Q C \perp A D Q F H R H V Q$ HRK RSSAAQLTAARLKATGDE HQRTMW NoXa PAELEVECATQLRR GDK NERQKL PUMA EEQWARE I AQLRR ADD NAQYER

Figure 1.6 Sequence Alignment of BH3 Domains from BH3-only Proteins. Residues completely and partially conserved are colored magenta and yellow, respectively. 

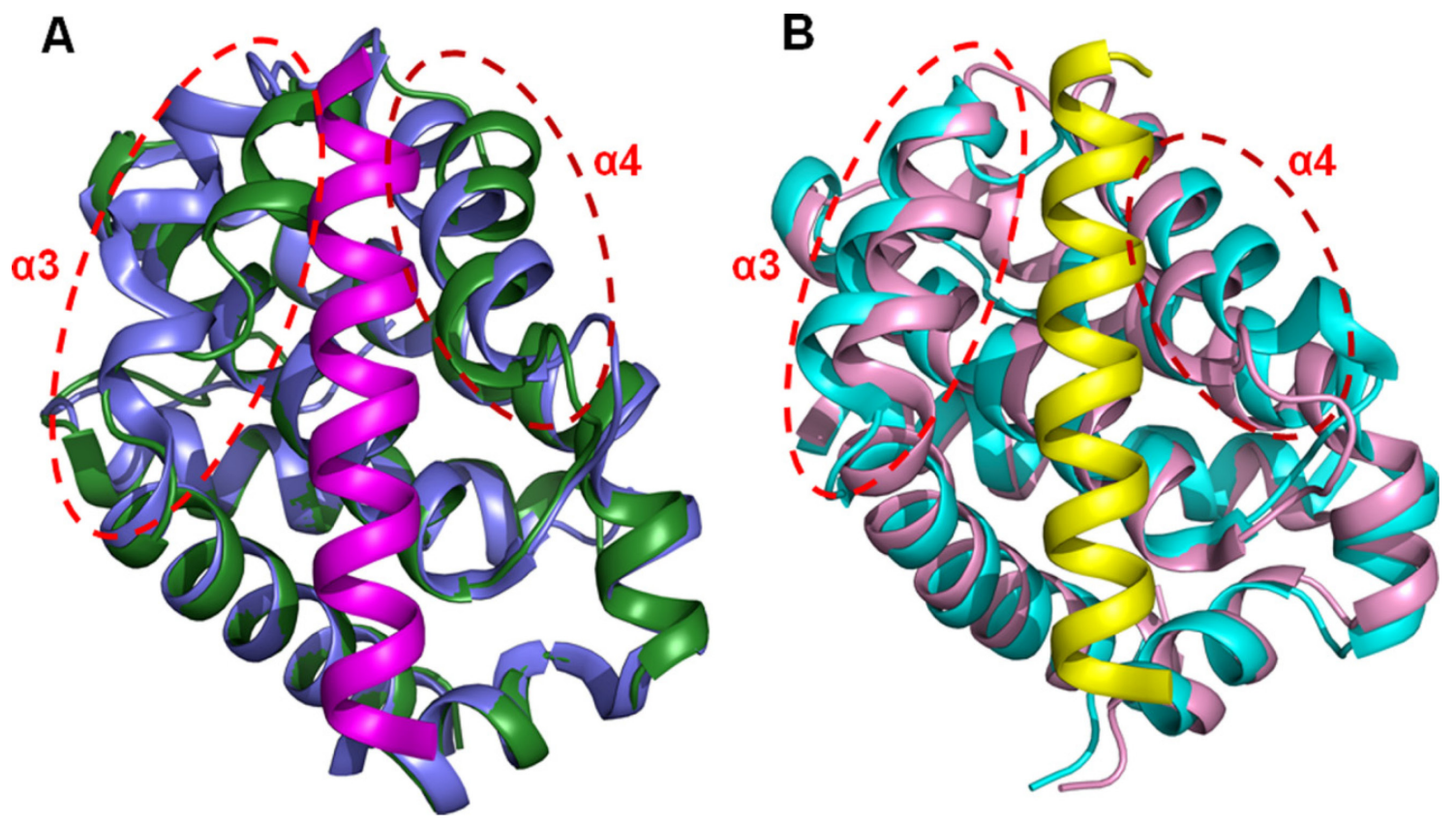

Figure 1.7 Structural Comparison of Apo Anti-apoptotic Proteins Compared with the Respective BH3 Domain-bound Forms.

(A) Overlay of apo BCL-xL (PDB ID:1LXL) and the BCL-xL·BIM complex (PDB ID:3FDL). Apo and BIM BH3 domain-bound BCL-xL are green and blue, respectively. The BIM BH3 domain is magenta. (B) Overlay of apo MCL-1 (PDB ID: 1WSX) and the MCL-1·BIM complex (PDB ID:2PQK). Apo and BIM BH3 domain-bound MCL-1 are pink and cyan, respectively. The BIM BH3 domain is yellow. Red dashed circles highlight alpha $(\alpha)$ helices three and four in the apo versus BIM BH3 domain-bound BCL-xL and MCL-1 structures (A-B). 
MCL-1 and BCL-xL and the BH3 domain-bound forms is the behavior of helices $\alpha 3$ and $\alpha 4$, which flank the groove and provide contact residues for the BH3 domain (Day et al., 2005). In BCL-xL, $\alpha 4$ undergoes a rotation to alter the $\alpha 4-\alpha 6$ helix crossing angle from approximately $60^{\circ}$ to almost parallel with $\alpha 6$, and the residues of $\alpha 3$ become less ordered on binding (Day et al., 2008). These ligand-induced changes in BCL-xL result in ligandinduced formation of the $\mathrm{BH} 3$ domain binding site by exposing residues that are otherwise buried in the closed conformation and creating space for the ligand (Figure 1.7A). In contrast, $\alpha 3$ of MCL-1 remains essentially unperturbed upon ligand binding (Figure 1.7B), and while $\alpha 4$ of MCL-1 does undergo a translation to widen the BC groove, a larger translation of the $\alpha 4$ helix was observed for BCL-xL binding (Day et al., 2008).

\section{The p53 Tumor Suppressor and Apoptosis}

The p53 tumor suppressor protein is arguably the most well-recognized tumor suppressor within the literature to date, and has even been dubbed guardian of the genome (Lane, 1992) because of its nodal position linking many different pathways that safeguard the integrity of genetic information in response to various genotoxic and nongenotoxic insults (Vogelstein et al., 2000). Loss of normal p53 function via inactivating mutations or an increase in p53 negative regulatory proteins is a hallmark of nearly all cancers. The tumor suppressor gene TRP53 encodes a transcription factor that regulates genes involved in apoptosis, cell cycle control, DNA repair, metabolism, and senescence (Vousden and Prives, 2009).

Cells that are insulted by oncogene activation, DNA damage, or other forms of stress stabilize the p53 protein by phosphorylation or other post-translational modifications (Bullock and Fersht, 2001) (Figure 1.8). Stabilized p53 accumulates in the nucleus and activates the expression of numerous pro-apoptotic genes including $A P A F-1$, BAX, PIG3, PUMA, Noxa CD95 (Fas), DR5/Killer, p53AIP1, Pidd, and Dram, to name only a few (Vousden and Prives, 2009). Silencing each of these genes in model systems leads to partial resistance to p53-induced apoptosis. The general view is that these genes govern the decision to live or die based on the cell type investigated and the applied death stimulus (Vousden and Prives, 2009).

\section{PUMA}

PUMA is a de-repressor BH3-only protein which collaborates with direct activator proteins to efficiently induce MOMP (Chipuk et al., 2008). Apoptotic signals trigger p53 levels to build within the nucleus where it serves as a transcription factor, rapidly up-regulating the expression of target genes including PUMA. As PUMA protein begins to amass within the cytoplasm, it subsequently translocates to the mitochondria where it initiates the mitochondrial apoptotic program via either sensitization or derepression, described above. There are two isoforms of PUMA, $\alpha$ and $\beta$. Importantly, both isoforms contain identical $\mathrm{BH} 3$ domain sequences, and aside from the sequences 


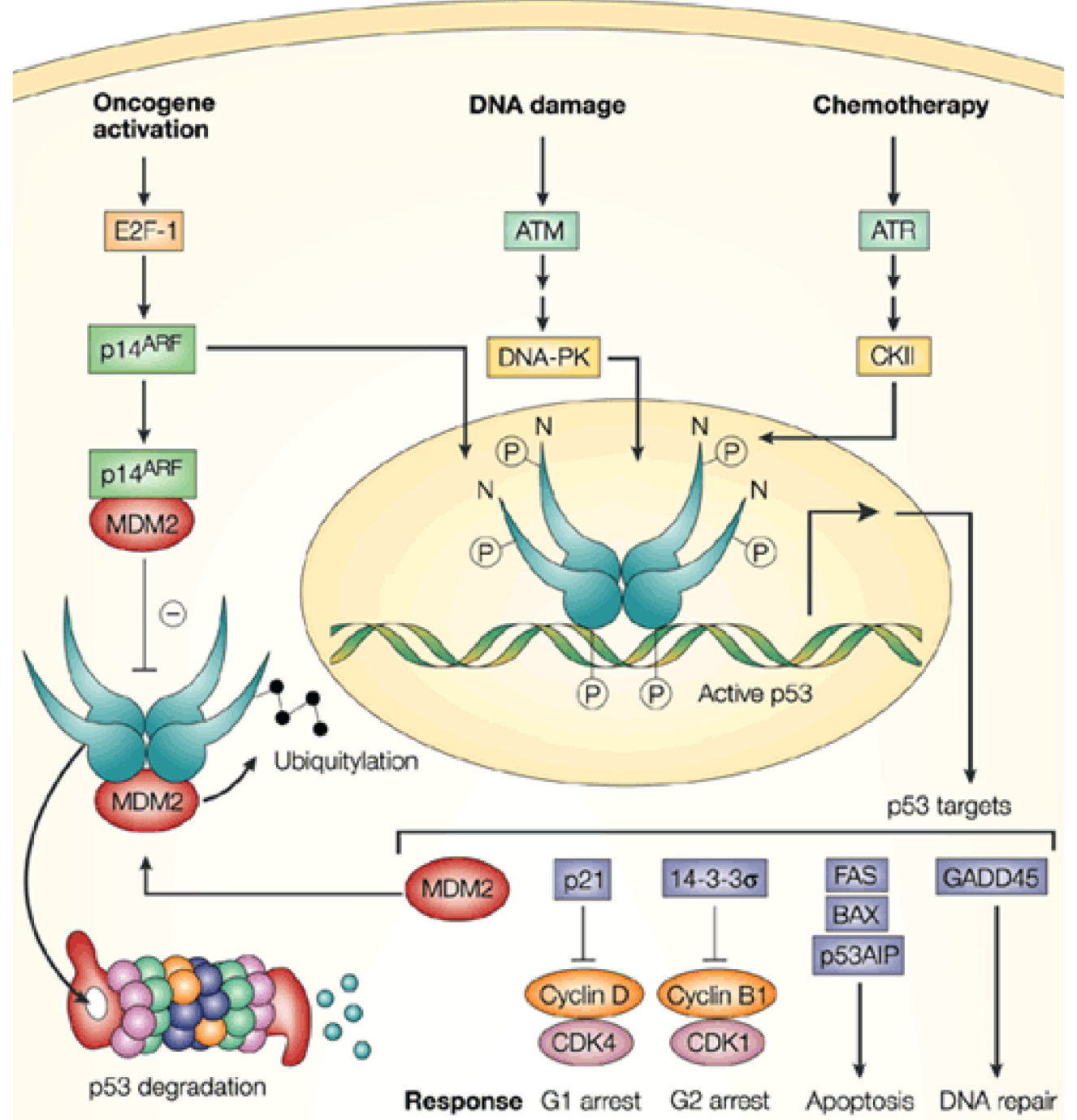

Figure 1.8 p53 Regulation.

In normal cells, $\mathrm{p} 53$-dependent transcription of $M D M 2$ promotes $\mathrm{p} 53$ degradation. Cellular stress, such as oncogene activation, induces p14 ${ }^{\mathrm{ARF}}$, which sequesters MDM2. In addition, DNA damage and chemotherapeutic agents activate protein kinases, such as ATM and ATR, which, through DNA-dependent protein kinase (DNA-PK) and casein kinase II (CKII), respectively, phosphorylate the amino terminus of p53 to prevent MDM2 binding, and the carboxyl terminus of p53 to increase sequence-specific DNA binding. These events increase p53 levels and activate the transcription of p53 target genes. p21 and 14-3-3 o promote growth arrest at the G1 and G2 DNA-damage checkpoints by inhibiting cyclin-dependent protein kinase (CDK) activity; FAS, BAX and p53AIP promote apoptosis if repair is not possible; and GADD45 promotes DNA repair. Reprinted with permission by Macmillan Publishers Ltd: Bullock, A.N., and Fersht, A.R. (2001). Rescuing the function of mutant p53. Nat Rev Cancer 1, 68-76. 
$\mathrm{N}$-terminal to the $\mathrm{BH} 3$ domain, the sequences of the isoforms are identical (Figure 1.9), and the kinetics of their upregulation following genotoxic stress are identical (Chipuk et al., 2008).

\section{Puma Knockout Mice Are Resistant to Apoptosis}

Loss of Puma results in resistance to numerous p53-dependent and -independent death stimuli in vivo indicating that this protein likely functions in numerous apoptotic networks (Jeffers et al., 2003; Villunger et al., 2003). Puma ${ }^{-/-}$mice were born at the expected Mendelian frequency, appeared normal and exhibited proper cellularity of the hematopoietic organs (Jeffers et al., 2003). Yet, numerous Puma ${ }^{-/-}$cell types (e.g., thymocytes, pre-B and mature B- and T-cells) were markedly resistant to genotoxic stress-induced apoptosis (e.g., $\gamma$-irradiation), similar to apoptosis resistance observed in $\operatorname{Trp}_{53^{-/}}$mice (Jeffers et al., 2003). PUMA may also regulate p53-independent pathways because $\mathrm{CD}^{+} 8^{+}$thymocytes derived from Puma ${ }^{-/-}$animals displayed resistance to dexamethasone and staurosporine, two known inducers of p53-independent apoptosis (Jeffers et al., 2003). This suggests that PUMA may participate in several death networks to sensitize cells to death stimuli that are regulated by diverse pro- and anti-apoptotic BCL-2 proteins.

\section{Apoptosis and Cancer}

Defects in apoptosis play important roles in tumor pathogenesis, allowing neoplastic cells to survive beyond their normally intended lifespan, subverting the need for exogenous survival factors, providing protection from hypoxia and oxidative stress as tumor mass expands, and allowing time for accumulative genetic alterations that deregulate cell proliferation, interfere with differentiation, promote angiogenesis, and increase cell motility and invasiveness during tumor progression (Reed, 1999). Apoptosis defects are recognized as an important complement to proto-oncogene activation, as many deregulated oncoproteins that drive cell division also trigger apoptosis (Green and Evan, 2002). Similarly, defects in DNA repair and chromosome segregation normally trigger apoptosis as a defense mechanism for eradicating genetically unstable cells, providing opportunities for selection of progressively aggressive clones (Ionov et al., 2000). Apoptosis defects also facilitate metastasis by allowing epithelial cells to survive in a suspended state with no extracellular matrix attachment (Frisch and Screaton, 2001).

\section{PUMA and Cancer}

E $\mu$-myc transgenic mice develop B-cell lymphomas with $100 \%$ incidence within the first year of life (Egle et al., 2004). This lymphomagenesis is accelerated by loss ofp53 or loss of ARF, an important protein which activates expression of p53 due to uncontrolled mitogenic signaling by inhibiting MDM2 (Eischen et al., 1999a).

Consistently, loss of a single copy of Puma drastically accelerates c-myc-induced B-cell 


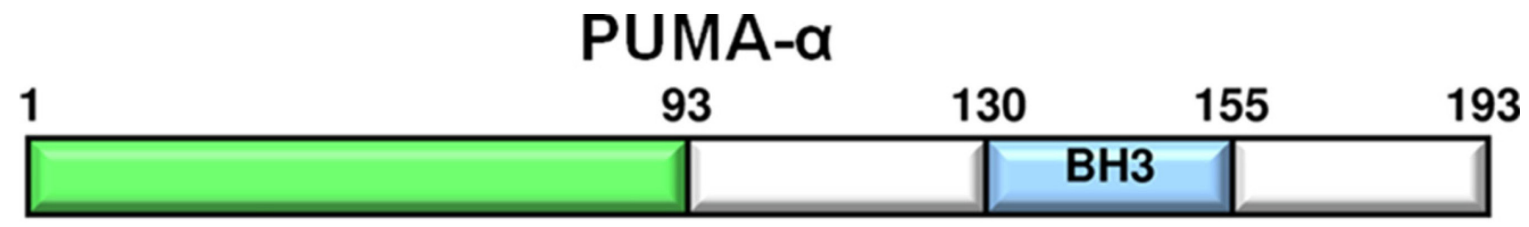

\section{PUMA- $\beta$}

\begin{tabular}{|l|l|l|l|l|}
\hline & 31 & 68 & 92 & \\
\hline & & $\mathrm{BH} 3$ & \\
\hline
\end{tabular}

Figure 1.9 Comparison of PUMA Alpha and Beta Isoforms.

Other than the extreme amino- (N-) terminus (residues 1-93 and 1-31 for the alpha and beta isoforms, respectively), both isoforms share identical sequences Numbers above each schematic represent amino acid numbers for the respective isoforms. The N-termini of PUMA alpha and beta are shown as green and magenta boxes, respectively. 
lymphomagenesis (Labi et al., 2006). When hematopoietic stem cells from $E \mu-m y c$ transgenic mice infected with a Puma-specific shRNA construct were transferred into normal recipients, lymphomas also arose with a drastically reduced latency, and disease onset was comparable to that observed in the absence of p53 (Hemann et al., 2004). Interestingly, the E $\mu$-myc lymphomas that carry a Puma-specific shRNA maintained wild type p53 status and could undergo cell cycle arrest in response to $\gamma$-irradiation (Hemann et al., 2004). In contrast, p53 is frequently inactivated or lost in $E \mu-m y c$ lymphomas (Eischen et al., 1999b), therefore it appears that the pressure to lose p53 is obviated by loss of PUMA. Importantly, in a recent landmark study, an enormous collection of human cancer samples were screened for somatic copy-number alterations and found PUMA deletion to be the most frequently deleted pro-apoptotic BCL-2 family gene (Beroukhim et al., 2010). Together, these findings strongly suggest that when cell cycle control is deregulated (e.g., by c-myc overexpression), the pro-apoptotic function of p53 is its most critical activity in tumor suppression (Labi et al., 2006).

The chromosomal locus 19q13.3, harboring the PUMA gene is frequently lost in human gliomas, neuroblastomas, and certain B-cell lymphomas (Mora et al., 2001; Shimazaki et al., 2000; Yong et al., 1995). It has also been reported that PUMA expression decreases during the development of malignant cutaneous melanoma (Karst et al., 2005). In addition, complete silencing of PUMA via epigenetic hypermethylation was found in approximately $40 \%$ of primary human Burkitt's lymphoma (Garrison et al., 2008).

There is also compelling evidence for the involvement of PUMA in p53-mediated renal cell apoptosis during cisplatin-induced nephrotoxicity (Jiang et al., 2006).

Therapeutically, this finding suggests the possibility of renal protection by blocking p53 or PUMA during cisplatin treatment. Further, it has also been established that PUMA is an essential mediator of cardiomyocyte death in response to Infarction/Reperfusion (I/R) (Toth et al., 2006). The study proposes a link between PUMA and I/R-associated myocardial dysfunction. On the basis of the marked resistance of PUMA deficient hearts to I/R, PUMA holds promise as a potential target for therapeutic intervention (Toth et al., 2006).

\section{BCL-xL and Cancer}

The noncompliant nature of cancer cells (e.g. exhibiting oncogene activation, cell cycle checkpoint violation, and genomic instability to name but a few transforming features) constitutively provokes death signals by engaging the apoptotic program, yet still these rogue cells manage to evade cell death. The road to tumorigenesis must, therefore, require apoptotic deficiency as a component. In support of this notion, it has been established that numerous cancer cell types become addicted to BCL-xL (and other anti-apoptotic members of the BCL-2 family) (Certo et al., 2006; Green and Evan, 2002).

Over-expression of BCL-xL occurs in more than half of all cancers (Amundson et al., 2000), rendering tumor cells resistant to numerous apoptotic stimuli, including 
oncogene expression and most cytotoxic anticancer drugs. Indeed, in the study which screened somatic copy-number alterations, mentioned above regarding PUMA, found $B C L-x L$ amplification to be the most frequently overexpressed $\mathrm{BCl}-2$ family gene (Beroukhim et al., 2010). Thus, understanding how BCL-xL functions, and relationships between its structural, biophysical, biochemical, and functional properties, is critical for understanding the role of BCL-xL in cancer.

\section{Cytoplasmic p53 and Its Transcription-Independent, Pro-apoptotic Function}

Early studies which employed temperature sensitive or DNA binding mutants of p53 suggested that it also functions in a transcription-independent manner to trigger apoptosis (Caelles et al., 1993; Haupt et al., 1995). This transcription-independent, proapoptotic effect was later observed with wild type p53 in the absence of nuclei (Chipuk et al., 2003). Insights into the mechanism(s) responsible for this transcription-independent function came with observations that cytosolic p53 binds to anti-apoptotic BCL-2 family proteins and can also function as a direct activator of the pro-apoptotic BCL-2 effector proteins BAK and BAX to promote MOMP, cytochrome c release and apoptosis (Chipuk et al., 2005b; Chipuk et al., 2004; Chipuk et al., 2003; Leu et al., 2004; Mihara et al., 2003).

The p53 protein is constitutively expressed but rapidly degraded by an interaction with one or more of its transcriptional targets, such as the ubiquitin E3 ligase MDM2 (Ashcroft et al., 2000). A variety of signals resulting from DNA damage, hypoxia, oncogene activation or other cellular stresses cause post-translational modification of p53, which disrupts its interaction with MDM2 and blocks p53 degradation (Kruse and Gu, 2009; Vogelstein et al., 2000). Upon stabilization, p53 accumulates in the nucleus and regulates gene expression, including transactivation of Puma. Concomitantly, p53 accumulates in the cytosol where it is sequestered by anti-apoptotic BCL-2 proteins, especially BCL-xL (Chipuk et al., 2005b; Chipuk et al., 2004; Mihara et al., 2003). Both PUMA protein and the PUMA BH3 domain peptide (PUMA ${ }^{\mathrm{BH} 3}$ ) uniquely disrupt this interaction, releasing cytoplasmic p53 to activate MOMP and promote apoptosis (Chipuk et al., 2008; Chipuk and Green, 2005).

\section{Project Summary}

Apart from its nuclear role as a transcription factor, cytosolic p53 regulates apoptosis through interactions with the BCL-2 family of proteins. While lacking a classic BH3 domain, p53 none the less functions as a "direct activator" of apoptosis (Chipuk et al., 2004). By default, cytosolic p53 is sequestered in inactive complexes by BCL-xL. However, when expressed, PUMA binds BCL-xL and releases sequestered p53. Released $\mathrm{p} 53$, in turn, directly interacts with BAX triggering a cascade of events leading to permeabilization of the outer mitochondrial membrane, cytochrome c release, and, ultimately, apoptosis. Importantly, PUMA is the only de-repressor BH3-only protein that releases p53 from BCL-xL, unleashing p53's full apoptotic potential. 
The molecular mechanism by which PUMA disrupts the BCL-xL·p53 complex to promote MOMP and apoptosis remains unclear. Previous structural studies revealed that the residues of p53 responsible for binding to BCL-xL do not occupy the canonical hydrophobic groove of BCL-xL akin to other pro-apoptotic BCL-2 proteins (e.g., BAK and BIM) (Petros et al., 2004a; Sot et al., 2007; Tomita et al., 2006), suggesting that PUMA does not simply compete with p53 for BCL-xL. Therefore, we explored the BCL-xL·PUMA interaction at the structural, biochemical, and cellular levels to elucidate how PUMA influences the binding of p53, releasing it to induce MOMP and apoptosis. 


\title{
CHAPTER 2. REAGENTS AND EXPERIMENTAL PROCEDURES
}

\section{Reagents}

\begin{abstract}
$\mathrm{BH} 3$ domain peptides: human $\mathrm{BAD}^{\mathrm{BH} 3}, \mathrm{BAK}^{\mathrm{BH} 3}, \mathrm{BAX}^{\mathrm{BH} 3}, \mathrm{BID}^{\mathrm{BH} 3}, \mathrm{BIM}^{\mathrm{BH} 3}$, $\mathrm{HRK}^{\mathrm{BH} 3}$, Noxa ${ }^{\mathrm{BH} 3}$ PUMA $^{\mathrm{BH} 3}$, and $\mathrm{PUMA}^{\mathrm{BH} 3 \mathrm{~W} 71 \mathrm{~A}}$ ( $>98 \%$ purity, Anaspec). Fluoresceinlabeled $\mathrm{PUMA}^{\mathrm{BH}}$ and alanine variants as well as the PUMA-BAD chimeric BH3 domain peptides ( $>98 \%$ purity, Hartwell Center for Bioinformatics and Biotechnology, St. Jude Children's Research Hospital) were synthesized using standard Fmoc-based chemistry. Fluorescein- peptide labeling included an additional amino terminal fluorescein conjugation step. Baculoviral expressed p53 (p53 ${ }^{\mathrm{Bac}}$ ) was purchased from Protein One. BCL-2 $\triangle$ C and C8-BID were purchased from R\&D Systems. p53 immunopurified from MCF7 cells (p53 ${ }^{\text {UVIP }}$ ) was made as described (Chipuk et al., 2004). Human full-length monomeric BAX was purified as described (Suzuki et al., 2000). pCMVneoBam-FLAGPUMA W71A, PUMA W71A in pET-30 (Novagen), BCL-xL $\Delta C$ E7C, BCL-xL $\Delta C$ H113A, BCL-xL $\Delta$ C N175C, and BCL-xL $\Delta$ C V135C,W181C (all in pET28a) were generated using the Stragene Quick Change Kit (Stratagene) with mutagenic oligonucleotides primers (Table A.1). All protein expression vector details are listed in
\end{abstract} Table A.2.

\section{Recombinant Proteins}

Recombinant human, full-length PUMA beta (hereafter referred to as PUMA) was expressed in E. coli strain BL21(DE3) (Novagen) using the pET-30a-vector. PUMA was expressed as a Histadine tag fusion by the addition of $1 \mathrm{mM}$ IPTG. Cell pellets were resuspended in lysis buffer (50 mM Tris, $\mathrm{pH} 8.0,500 \mathrm{mM} \mathrm{NaCl}, 5 \mathrm{mM}$ imidazole, 5.0 $\mathrm{mM}$ TCEP) plus EDTA-free protease inhibitor tablets (one tablet per $50 \mathrm{ml}$ buffer) (Roche) and affinity purified with Ni-NTA Superflow resin (Qiagen). Fractions containing PUMA were pooled and further purified by reversed-phase high performance liquid chromatography (HPLC) using a semi-preparative C4 column (Vydac) and an acetonitrile linear gradient (Figure A.1). HPLC fraction two containing active PUMA was lyophilized and stored at $-80^{\circ} \mathrm{C}$. Functional activity of recombinant PUMA protein was assessed by an in vitro cytochrome c release assay (Figure 2.1). The purity of the lyophilized protein was greater than $95 \%$ based on SDS-PAGE (Figure 2.2).

Recombinant human PUMA W71A, PUMA ${ }^{\mathrm{N}}$ (PUMA residues 1-67) and PUMA ${ }^{\mathrm{C}}$ (PUMA residues 93-131) were expressed and purified to greater than 95\% purity based on SDS-PAGE using the same procedures as described above for wild type PUMA beta (Figure 2.2).

Recombinant human BCL-xL $\Delta \mathrm{C}$ (residues 1-209) was produced in E. coli strain BL21(DE3) using a pET-28 vector (Novagen). BCLxL $\Delta \mathrm{C}$ was expressed as a hexa histidine tagged fusion by the addition of $1 \mathrm{mM}$ IPTG. Cells were resuspended in lysis buffer (50 mM Tris $\mathrm{pH} 8.0,500 \mathrm{mM} \mathrm{NaCl}, 5 \mathrm{mM}$ imidazole) plus one EDTA-free 

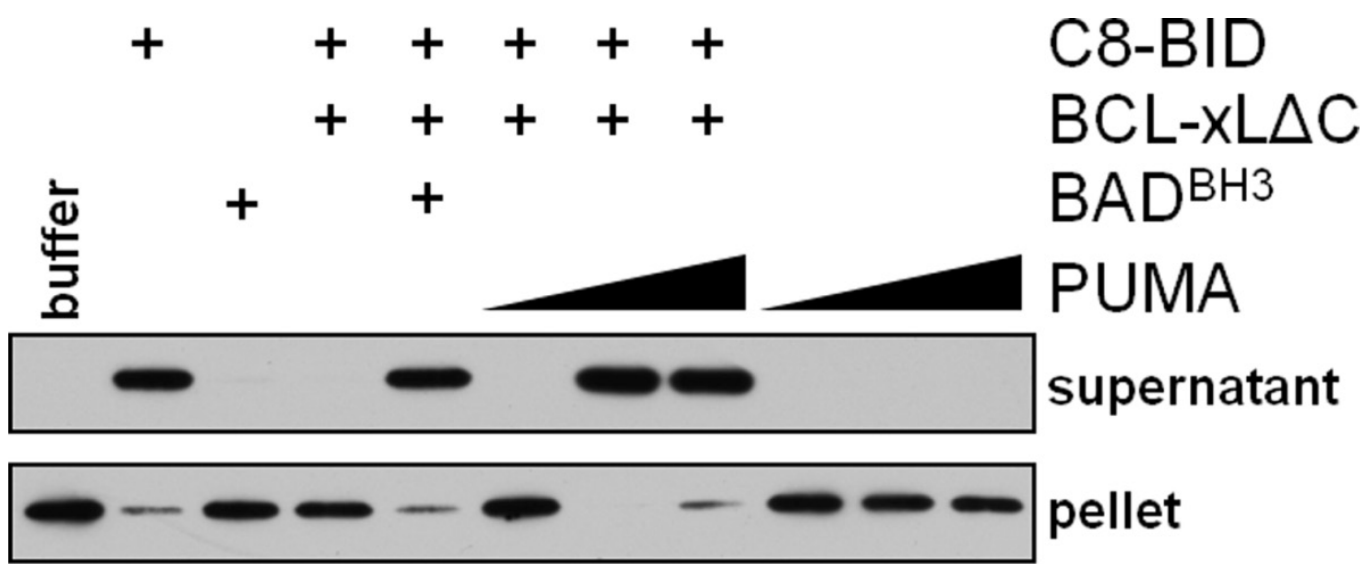

WB: cytochrome c

\section{C8-BID: $1 \mathrm{nM}$ \\ BCL-XL: $50 \mathrm{nM}$ \\ $\mathrm{BAD}^{\mathrm{BH} 3}: 5 \mu \mathrm{M}$ \\ PUMA : 50, $500 \& 1000 \mathrm{nM}$}

Figure 2.1 Recombinant PUMA and BCL-xL Proteins Are Active.

Purified, murine liver mitochondria were treated with the indicated proteins before fractionation, SDS-PAGE, and western blot analysis for cytochrome c. C8-BID (caspase8-cleaved $\mathrm{BID}), \mathrm{BAD}^{\mathrm{BH} 3}$ (BAD BH3 domain peptide). 
Figure 2.2 SDS-PAGE Analysis of the Indicated Recombinant Proteins. 

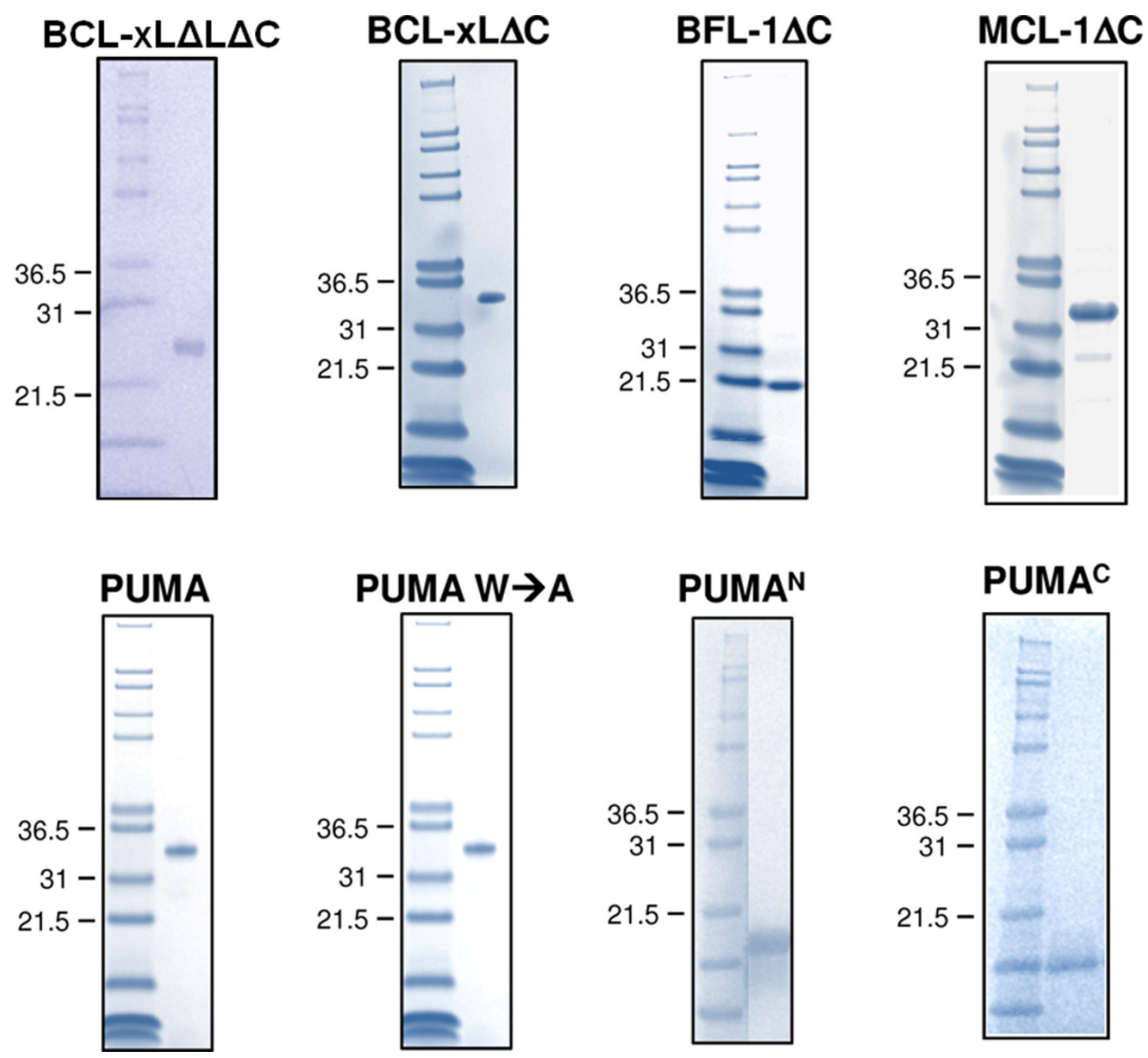

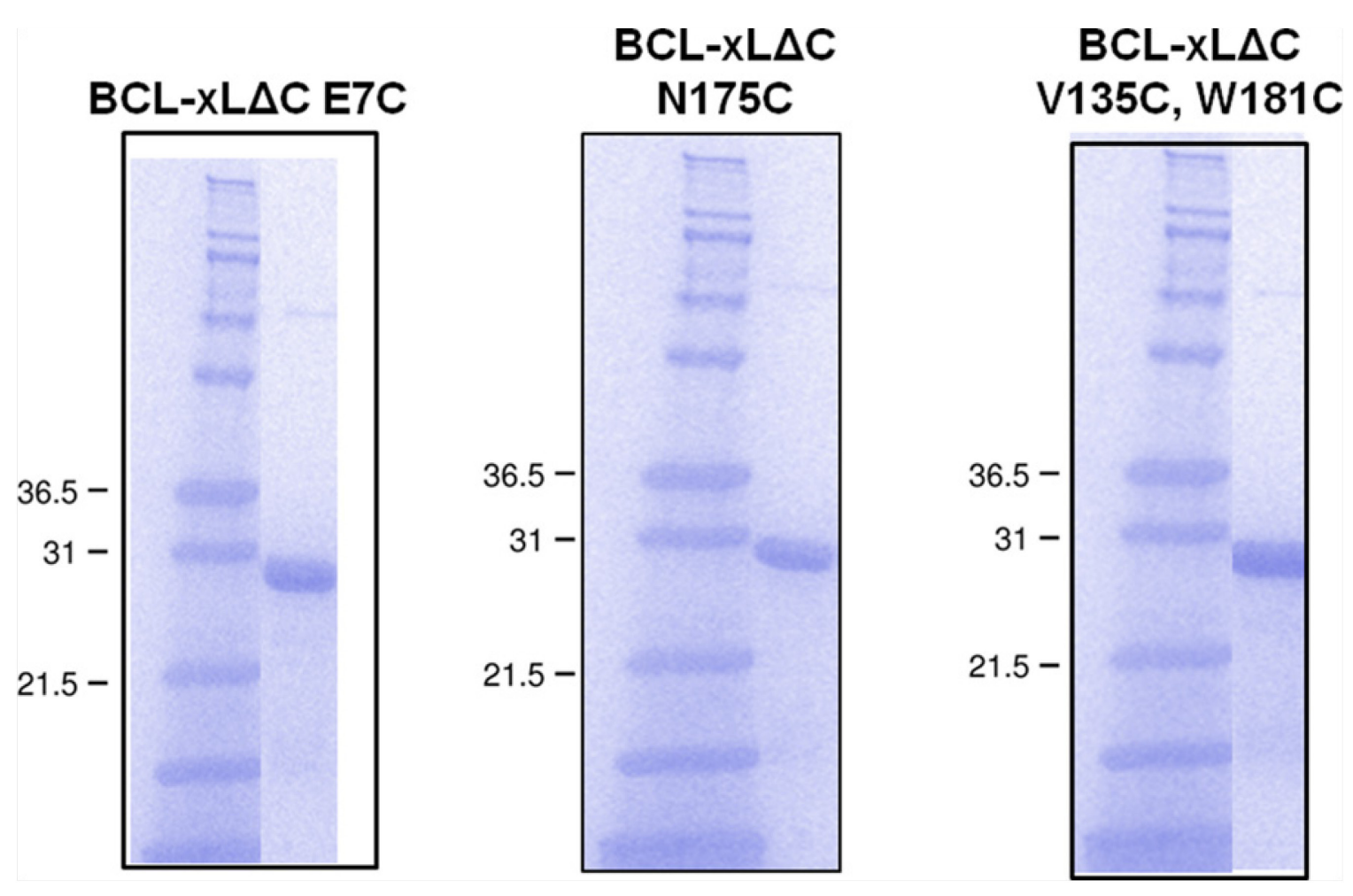

Figure 2.2 (Continued). 
protease inhibitor tablet per $50 \mathrm{ml}$ of buffer (Roche) and affinity purified with Ni-NTA Superflow resin (Qiagen). The N-terminal hexa histidine tag was cleaved with thrombin protease (1 unit protease/mg protein) and the protein was diluted tenfold with anion exchange buffer ( $50 \mathrm{mM}$ Tris, $\mathrm{pH}$ 8.0, $10 \mathrm{mM} \mathrm{NaCl})$. The diluted sample was loaded onto a HiTrap Q HP column (GE Healthcare) and eluted with a linear gradient of increasing $\mathrm{NaCl}$ concentration. Fractions containing BCL-xL $\Delta \mathrm{C}$ were further purified by Superdex 200 gel filtration (Amersham) in $50 \mathrm{mM}$ Tris $\mathrm{pH} 8.0$ and $300 \mathrm{mM} \mathrm{NaCl}$, and the protein was concentrated using an Amicon Ultra filtration device (Millipore).

Recombinant BCL-xL $\Delta C$ was purified to greater than $95 \%$ purity based on SDS-PAGE (Figure 2.2).

Human MCL-1 $\Delta \mathrm{N} \Delta \mathrm{C}$ was produced in $E$. coli strain BL21(DE3) (Novagen) using the pET-151 vector and expressed as a hexa histidine tag fusion by the addition of $1 \mathrm{mM}$ IPTG. Cell pellets were resuspended in lysis buffer $(50 \mathrm{mM}$ Tris, $\mathrm{pH} 8.0,500 \mathrm{mM} \mathrm{NaCl}$, $5 \mathrm{mM}$ imidazole, $5.0 \mathrm{mM}$ TCEP) plus one EDTA-free protease inhibitor tablet per $50 \mathrm{ml}$ of buffer (Roche) and affinity purified with Ni-NTA Superflow resin (Qiagen) followed by gel filtration on a Superdex 200 preparative gel filtration column (Amersham). Recombinant MCL-1 $\triangle \mathrm{C}$ was purified to greater than $95 \%$ purity based on SDS-PAGE (Figure 2.2).

Human BFL-1 $\triangle \mathrm{C}$ was produced in E. coli strain BL21(DE3) (Novagen) using the pGEX-6P3 vector (GE Healthcare) and expressed as a glutathione S-transferase (GST) tag fusion by the addition of $0.2 \mathrm{mM} \mathrm{IPTG}$ and cultured at $18^{\circ} \mathrm{C}$ for $24 \mathrm{hr}$. Cell pellets were resuspended in lysis buffer (PBS) plus protease inhibitor mixture (Roche) and affinity purified with Glutathione Sepharose Fast Flow resin (GE Healthcare). The GST tag was cleaved by PreScission protease (GE Healthcare) and removed by a second GST affinity step. BFL-1 $\Delta \mathrm{C}$ was further purified by gel filtration on a Superdex 200 preparative gel filtration column (Amersham). Recombinant BFL-1 $\Delta \mathrm{C}$ was purified to greater than $95 \%$ purity based on SDS-PAGE (Figure 2.2).

BCL-xL $\Delta \mathrm{L} \Delta \mathrm{C}, \mathrm{BCL}-\mathrm{xL} \Delta \mathrm{C}$ E7C, BCL-xL $\Delta \mathrm{C}$ N175C, and BCL-xL $\Delta \mathrm{C}$ V135C,W181C were expressed and purified to greater than $95 \%$ purity based on SDSPAGE (Figure 2.2) using the same methods described above for BCL-xL $\Delta C$.

\section{Calorimetric Studies}

Most ITC experiments were done using a VP-ITC (Microcal) calorimeter. Titrations consisted of a preliminary $2 \mu \mathrm{l}$ injection of $100 \mu \mathrm{M}$ PUMA or various BH3 domain peptides, followed by several injections of $6 \mu \mathrm{l}$ of either PUMA or BH3 domain peptide into a solution of $10 \mu \mathrm{M}$ BCL-xL $\Delta \mathrm{C}$ monomer, BCL-xL $\Delta \mathrm{C}$ apo-dimer, MCL$1 \Delta \mathrm{C}$, or BFL- $1 \Delta \mathrm{C}$. Experiments were conducted at $25^{\circ} \mathrm{C}$ in $20 \mathrm{mM}$ Tris, $\mathrm{pH} 7.4,100$ $\mathrm{mM} \mathrm{NaCl}$ and $1 \mathrm{mM}$ TCEP. The time interval between injections was $300 \mathrm{sec}$. The instrument was regularly calibrated using water into water titratrions. Thermodynamic parameters were obtained using Origin software (OriginLab) using routines provided by the manufacturer. Heats of reaction were corrected for the effect of dilution by injection 
of protein or peptide titrant from the syringe into ITC buffer alone followed by subsequent subtraction of these heats from the original experiment.

ITC experiments for BCL-xL $\Delta \mathrm{C}$ and $\mathrm{p} 53^{\mathrm{Bac}}$ were done using an ITC-200 (Microcal) calorimeter. Titrations consisted of a preliminary $0.5 \mu 1$ injection of BCL$\mathrm{xL} \Delta \mathrm{C}$ or BCL-xL $\Delta \mathrm{C}$ apo-dimer, followed by several injections of $2 \mu \mathrm{l}$ of either BCL$\mathrm{xL} \Delta \mathrm{C}$ or BCL-xL $\Delta \mathrm{C}$ apo-dimer into a solution of $\mathrm{p} 53^{\mathrm{Bac}}$. Experiments were conducted at $15{ }^{\circ} \mathrm{C}$ in $20 \mathrm{mM}$ Tris, $\mathrm{pH} 7.5,50 \mathrm{mM} \mathrm{NaCl}$ and $1 \mathrm{mM}$ TCEP. The time interval between injections was $180 \mathrm{sec}$. Despite extensive, simultaneous dialysis of all proteins against ITC buffer to remove the glycerol from $\mathrm{p} 53^{\mathrm{Bac}}$ (provided in $20 \%$ glycerol by the manufacturer), a buffer mismatch remained between the final protein samples. A control experiment was performed in which BCL-xL $\Delta C$ was titrated into ITC buffer to measure the heat of dilution and obtain a control isotherm. A point-by-point subtraction of the control isotherm from the experimental binding isotherm was performed to obtain final isotherms representative of heat changes produced by BCL-xL $\Delta C$ or BCL-xL $\Delta C$ apo-

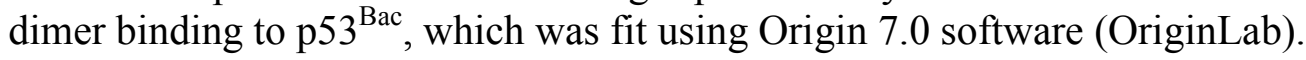

\section{Circular Dichroism Spectropolarimetry}

CD data were collected on an Aviv 62A DS spectropolarimeter. Far-UV CD wavelength scans (260-195 nm) were performed with $5 \mu \mathrm{M}$ PUMA protein or $50 \mu \mathrm{M}$ $\mathrm{PUMA}^{\mathrm{BH} 3}$ in $20 \mathrm{mM}$ potassium phosphate $\mathrm{pH} 7.0$ using the following standard measurement parameters: wavelength, $195-260 \mathrm{~nm}$; step resolution, $0.5 \mathrm{~nm}$; speed, 20 $\mathrm{nm} / \mathrm{min}$; accumulations, 10; response, $1 \mathrm{sec}$; bandwidth, $1 \mathrm{~nm}$; path length, $0.1 \mathrm{~cm}$. Temperature was maintained at $25{ }^{\circ} \mathrm{C}$ with a Peltier thermoelectric device. Molar ellipticity was calculated using the following equation:

$$
[\Theta]=\left[(100)\left(\Theta_{o b s}\right)\right] /[(c)(l)]
$$

where $[\Theta]$ is molar ellipticity, $\Theta_{o b s}$ is observed ellipticity, $c$ is molar concentration, and $l$ is the cuvette pathlength in meters.

\section{NMR Spectroscopy}

${ }^{15} \mathrm{~N}$-labeled BCL-xL $\Delta \mathrm{L} \Delta \mathrm{C}$ and ${ }^{15} \mathrm{~N}$-labeled PUMA for NMR studies were expressed using a MOPS-based minimal media containing ${ }^{15} \mathrm{~N}-\mathrm{NH}_{4} \mathrm{Cl}$ (Lacy et al., 2004) and purified using the procedures described above for the respective unlabled proteins.

${ }^{15} \mathrm{~N}$-labeled BCL-xL $\Delta \mathrm{C}$ proteins were prepared at $0.3 \mathrm{mM}$ in $20 \mathrm{mM}$ sodium phosphate $\mathrm{pH} 7.0,3 \mathrm{mM}$ DTT, and $0.5 \mathrm{mM}$ EDTA. ${ }^{15} \mathrm{~N}$-labeled PUMA was prepared at $0.3 \mathrm{mM}$ in $20 \mathrm{mM}$ deuterated Tris (Cambridge), $\mathrm{pH} 7.1,150 \mathrm{mM} \mathrm{NaCl}$, and $5 \mathrm{mM}$ deuterated DTT (Cambridge). Data were acquired on Bruker $600 \mathrm{MHz}$ and $800 \mathrm{MHz}$ spectrometers equipped with cryogenically-cooled, triple-resonance single-axis gradient probes. One dimensional (1D) spectra were acquired at $30{ }^{\circ} \mathrm{C}$ using the zgesgp pulse sequence provided by Bruker (Hwang, 1995). Two dimensional (2D) ${ }^{1} \mathrm{H}-{ }^{15} \mathrm{~N}$ HSQC or TROSY 
spectra were acquired at 25 or $30{ }^{\circ} \mathrm{C}$ using the hsqcetf3gpsi and trosyf3gpphsi19 Bruker pulse sequences, respectively (Schleucher et al., 1994; Zhu et al., 1999). Spectra were processed using NMRPipe (Delaglio et al., 1995) and analyzed using TopSpin (provided by Bruker) and CARA software.

\section{Analytical Ultracentrifugation}

Dr. Amanda Nourse at the St. Jude Hartwell Center performed analytical ultracentrifugation (AUC) using the indicated purified proteins and $\mathrm{BH} 3$ domain peptides.

For sedimentation velocity experiments, BCL-xL $\Delta \mathrm{L} \Delta \mathrm{C}, \mathrm{BCL} x \mathrm{LL} \Delta \mathrm{L} \cdot \mathrm{BIM}^{\mathrm{BH} 3}$ and BCL-xL $\Delta \mathrm{L} \Delta \mathrm{C} \cdot \mathrm{PUMA}^{\mathrm{BH} 3}$ complex, all at approximately $1.1 \mathrm{mg} / \mathrm{ml}$ in $20 \mathrm{mM}$ Tris $\mathrm{pH} 7.5,100 \mathrm{mM} \mathrm{NaCl}$ were subjected to sedimentation velocity ultracentrifugation in a ProteomeLab XL-I analytical ultracentrifuge with a Beckman An-60 Ti rotor and cells containing sapphire windows and charcoal-filled Epon double-sector centerpieces (Beckman Coulter, Fullerton, CA). The density and viscosity of the buffer were calculated from their composition, and the partial specific volume and molecular weights of the protein and complexes were calculated based on their amino acid composition using the software SEDNTERP (Laue et al., 1992). All samples were dialysed against the ultracentrifugation buffer, and the dialysate was used as an optical reference.

Temperature equilibrium at $20^{\circ} \mathrm{C}$ was established in the instrument prior to the run over a period of at least $3 \mathrm{hr}$. Ultracentrifugation was performed at 60,000 rpm for $12 \mathrm{hr}$. Fringe displacement data at time intervals of $1.0 \mathrm{~min}$ were collected with the Rayleigh interference system and analysed with SEDFIT software using the model for continuous sedimentation coefficient distribution $\mathrm{c}(\mathrm{s})$ with deconvolution of diffusional effects (Schuck, 2000; Schuck et al., 2002). The sedimentation coefficient distribution $c(s)$ was calculated with maximum entropy regularization at a confidence level of $p=0.7$ and at a resolution of sedimentation coefficients of $n=120$. The positions of the meniscus and bottom, as well as time-invariant and radial noises, were fitted.

Sedimentation equilibrium experiments were performed on BCL-xL $\Delta \mathrm{L} \Delta \mathrm{C}, \mathrm{BCL}-$ $\mathrm{xL} \Delta \mathrm{L} \Delta \mathrm{C} \cdot \mathrm{BIM}^{\mathrm{BH} 3}, \mathrm{BCL}-\mathrm{xL} \Delta \mathrm{L} \Delta \mathrm{C} \cdot \mathrm{PUMA}^{\mathrm{BH} 3}$ complexes (sample concentrations of 1.0-16 $\mu \mathrm{M}$ ), and BCL-xL $\Delta \mathrm{C} \cdot \mathrm{PUMA}^{\mathrm{BH} 3}$ (sample concentrations of $0.19,0.42$, and $1.14 \mu \mathrm{M}$ ) in $20 \mathrm{mM}$ sodium phosphate $\mathrm{pH}$ 7.0, $100 \mathrm{mM} \mathrm{NaCl}$ buffer with a Beckman An-50 Ti rotor and cells containing quartz windows and charcoal-filled Epon double-sector center pieces (Beckman Coulter, Fullerton, CA). The density and viscosity of the buffer were calculated as described above. The buffer eluent of the size-exclusion column was used as an optical reference. Equilibrium was attained at $24 \mathrm{hr}$ at a rotor temperature of $4{ }^{\circ} \mathrm{C}$ at increasing speeds of 15,22 and 28 thousand rpm. $120 \mu \mathrm{L}$ of each sample was loaded into double-sector centrepieces and absorbance distributions recorded at 230 and $280 \mathrm{~nm}$ in $0.001 \mathrm{~cm}$ radial intervals with 20 replicates for each point. Global least squares modelling of all datasets (all concentrations, rotor speeds and wavelengths) were performed with the software SEDPHAT using a single species model as well as a reversible monomer-dimer self-association model (Balbo et al., 2007). 


\section{Crystallization, Data Collection, and Refinement}

Crystals of BCL-xL $\Delta \mathrm{C}$ were grown at $18{ }^{\circ} \mathrm{C}$ by hanging-drop vapor diffusion using equal volumes of $1.5 \mathrm{mM}$ protein and precipitant $(1.5 \mathrm{M}$ lithium sulfate, $0.1 \mathrm{M}$ trisodium citrate $\mathrm{pH}$ 5.6, and 2.0 M ammonium sulfate) (Figure 2.3). Crystals were cryoprotected in mother liquor supplemented with 30\% glycerol and flash-frozen in liquid nitrogen. Diffraction data (Figure 2.3) were collected at $100 \mathrm{~K}$ at the Southeast Regional Collaborative Access Team (SER-CAT) 22-ID synchrotron beamline using a $\mathrm{CCD}$ detector and a beam wavelength of $1.0 \AA$. Data were indexed and scaled using HKL2000 (Otwinowski and Minor, 1997). The structure was calculated to $2.1 \AA$. resolution by direct refinement using CNS (Brunger et al., 1998) and Xtalview (McRee and Israel, 2008). Data processing parameters are summarized in Table 2.1.

The BCL-xL $\Delta C \cdot P U M A^{\mathrm{BH} 3}$ complex was prepared by adding $\mathrm{PUMA}^{\mathrm{BH} 3}$ in a $1: 1.2$ molar ratio to $1.5 \mathrm{mM}$ BCL-xL $\Delta \mathrm{C}$ in $50 \mathrm{mM}$ Tris, $100 \mathrm{mM} \mathrm{NaCl} \mathrm{pH} \mathrm{7.5.} \mathrm{Crystals} \mathrm{were}$ grown at $18{ }^{\circ} \mathrm{C}$ by hanging-drop vapor diffusion mixing equal volumes of protein solution and precipitant $(0.2 \mathrm{M}$ calcium chloride, $0.1 \mathrm{M}$ sodium acetate $\mathrm{pH} 4.6$ and 20\% isopropanol) (Figure 2.3). The final pH of the drop was approximately 7.2. Crystals were cryoprotected in mother liquor supplemented with 30\% glycerol and flash-frozen in liquid nitrogen. Diffraction data (Figure 2.3) were collected at $100 \mathrm{~K}$ at the Southeast Regional Collaborative Access Team (SER-CAT) 22-ID synchrotron beamline using a $\mathrm{CCD}$ detector and a beam wavelength of $1.0 \AA$. Data were indexed and scaled using HKL2000 (Otwinowski and Minor, 1997). The structure of the BCL-xL $\Delta C \cdot P U M A^{\mathrm{BH} 3}$ complex was determined by molecular replacement using PHASER (Zwart et al., 2008). Model building was performed using COOT (Emsley and Cowtan, 2004), and iterative rounds of refinement were performed using CNS (Brunger et al., 1998) and Refmac using TLS refinement (Murshudov et al., 1997). Data processing parameters are summarized in Table 2.2. All structural figures were generated with PyMOL (www.pymol.org).

\section{Fluorescence Spectroscopy}

The affinity of fluorescein-labeled PUMA ${ }^{\mathrm{BH} 3}$ for BCL-xL $\Delta \mathrm{C}$ was determined by fluorescence anisotropy using a Fluorolog-3 spectrofluorometer (Jobin Yvon Inc). All experiments were performed at $30{ }^{\circ} \mathrm{C}$ with continuous stirring. Dilution buffer for all protein and peptide stocks was $120 \mathrm{mM}$ sodium phosphate $\mathrm{pH}$ 7.5. The concentrations of the DMSO peptide stock solutions were $0.5-1.5 \mathrm{mM}$, as determined by tryptophan absorbance at $280 \mathrm{~nm}$ or amino acid analysis. The final DMSO concentration for all samples was less than $1 \%$. The binding affinities of peptides to BCL-xL $\Delta \mathrm{C}$ were measured from the anisotropy of fluorescein fluorescence emission as a function of increasing BCL-xL $\Delta \mathrm{C}$ concentration (Table A.3). A five min time interval was used to allow every addition of BCL $\Delta \mathrm{C}$ to reach equilibrium. The excitation and emission wavelengths were 494 and $525 \mathrm{~nm}$, respectively. The grating factor was set to $0.5 \mathrm{~mm}$ to reduce noise. All data points represent the mean of at least two independent experiments. Fluorescence anisotropies were converted to fraction of ligand bound (Lakowicz, 1983) using the following equation: 
A

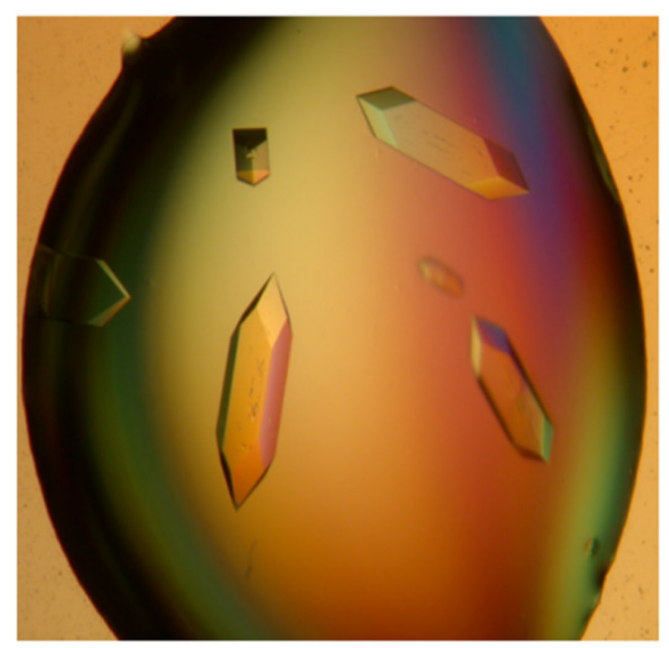

C

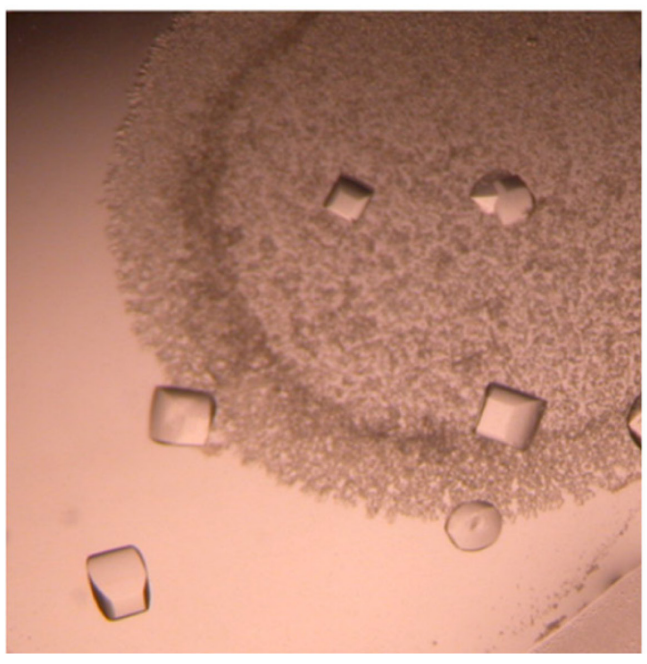

B

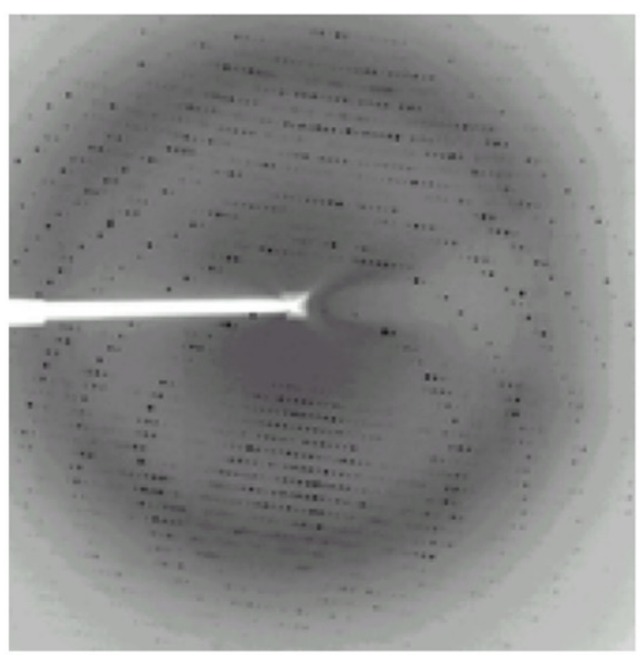

D

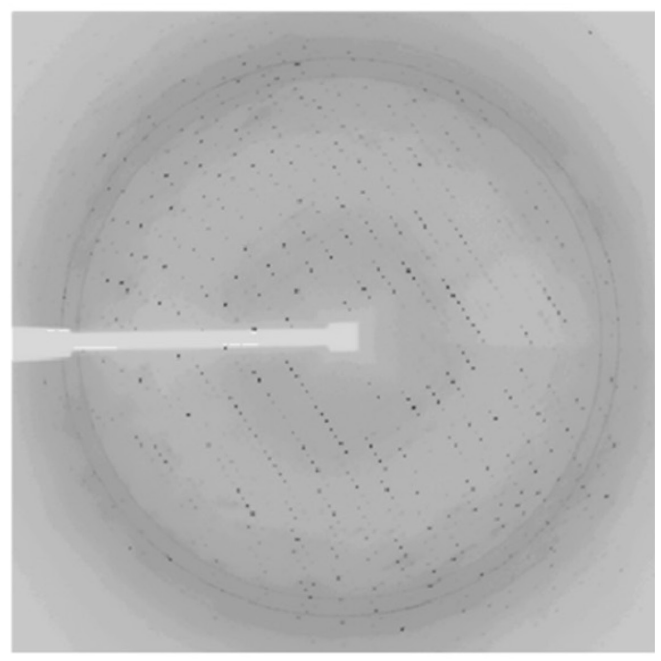

\section{Figure 2.3 Protein Crystals and Respective Diffraction Data Images.}

(A) Crystals of BCL-xL $\Delta C$ prior to harvesting and $\mathrm{x}$-ray diffraction. (B) Representative BCL-xL $\Delta C$ crystal x-ray diffraction image taken at $\Phi=90^{\circ}$. (C) Crystals of the BCL$\mathrm{xL} \triangle \mathrm{C} \cdot \mathrm{PUMA}^{\mathrm{BH} 3}$ complex prior to harvesting and $\mathrm{x}$-ray diffraction. (D) Representative $\mathrm{BCL}-\mathrm{xL} \Delta \mathrm{C}$ crystal x-ray diffraction image taken at $\Phi=90^{\circ}$. 
Table 2.1 BCL-xL $\Delta C$ Structure Data and Refinement Statistics.

\begin{tabular}{ll}
\hline Data & BCL-xLAC \\
\hline Data Collection & Values \\
Wavelength $(\AA)$ & 1.0 \\
Space Group & $\mathrm{P} 4{ }_{1} 2_{1} 2$ \\
Cell Dimension & \\
$\quad a, b, c(\AA)$ & $63.1,63.1,111.0$ \\
$\quad \alpha, \beta, \gamma\left({ }^{\circ}\right)$ & $90.0,90.0,90.0$ \\
Resolution $(\AA)$ & $50.0-2.1$ \\
$\mathrm{R}_{\text {sym }}{ }^{2}(\%)$ & $8.5(43.7)$ \\
$\mathrm{I} / \sigma \mathrm{I}$ & $49.8(4.0)^{\mathrm{a}}$ \\
Completeness & $98.9(96.8)$ \\
Redundancy & $16.0(10.9)$ \\
Data Refinement & \\
Resolution $(\AA)$ & $50.0-2.1$ \\
No. Reflection & 13194 \\
$\mathrm{R}_{\text {work }} / \mathrm{R}_{\text {free }}{ }^{\mathrm{b}}$ & $.225 / .248$ \\
No. Atoms & \\
Protein & 1153 \\
$\quad$ Water & 48 \\
Average B-factors $\left(\AA^{2}\right)$ & 38 \\
R.M.S. Deviations & 38 \\
Bond Lengths & 0.006 \\
Bond Angles & 1.1 \\
\hline
\end{tabular}

${ }^{\mathrm{a}}$ Numbers in parentheses refer to the highest resolution shell.

${ }^{\mathrm{b}} R_{\text {work }}=\mathrm{S} 11$ Fobs $1-1 F_{\text {calc }} 1 / \mathrm{S} 1 F_{\text {obs }} 1$ where $\mathrm{F}_{\text {obs }}$ and $\mathrm{F}_{\text {calc }}$ are observed and model structure factors, respectively.

$\mathrm{R}_{\text {free }}$ was calculated using a randomly selected set (5\%) of reflections. 
Table 2.2 BCL-xL $\Delta C \cdot P U M A^{\mathrm{BH} 3}$ Structure Data and Refinement Statistics.

\begin{tabular}{ll}
\hline Data & BCL-xLAC·PUMA ${ }^{\text {BH3 }}$ \\
\hline Data Collection & \\
Wavelength $(\AA)$ & 1.0 \\
Space group & $\mathrm{P}_{2} 22$ \\
Cell Dimension & \\
$\quad a, b, c(\AA)$ & $94.8,94.8,111.5$ \\
$\quad \alpha, \beta, \gamma\left({ }^{\circ}\right)$ & $90.0,90.0,90.0$ \\
Resolution $(\AA)$ & $50.0-2.9$ \\
$\mathrm{R}_{\text {sym }}{ }^{2}(\%)$ & $9.4(38)$ \\
$\mathrm{I} / \sigma \mathrm{I}$ & $38.4(3.28)$ \\
Completeness & $95.6(65.5)$ \\
Redundancy & $21.7(8.8)$ \\
Refinement & \\
Resolution $(\AA)$ & $43.6-2.9$ \\
No. Reflection & 10731 \\
$\mathrm{R}_{\text {work }} / \mathrm{R}_{\text {free }}{ }^{\mathrm{b}}$ & $.231 / .281$ \\
No. Atoms & \\
$\quad$ Protein & 349 \\
$\quad$ Water & 6 \\
Average B-factor $\left(\AA^{2}\right)$ & 73 \\
R.M.S. Deviations & \\
Bond Lengths & 0.011 \\
Bond Angles & 1.2 \\
\hline
\end{tabular}

${ }^{a}$ Numbers in parentheses refer to the highest resolution shell.

${ }^{\mathrm{b}} R_{\text {work }}=\mathrm{S} 11$ Fobs $1-1 F_{\text {calc }} 1 / \mathrm{S} 1 F_{\text {obs }} 1$ where $\mathrm{F}_{\text {obs }}$ and $\mathrm{F}_{\text {calc }}$ are observed and model structure factors, respectively.

$\mathrm{R}_{\text {free }}$ was calculated using a randomly selected set (5\%) of reflections. 


$$
F_{b}=\left(r-r_{\text {free }}\right) /\left[\left(r_{\text {bound }}-r\right)(q)+r-r_{\text {free }}\right]
$$

where $F_{b}$ is the molar fraction of bound ligand, $r$ is the anisotropy value at a given titration point, $r_{\text {free }}$ is the anisotropy value of free ligand at a given titration point, $r_{\text {bound }}$ is the anisotropy value of bound ligand at a given titration point, and $q$ is the ratio of fluorescence intensities of the bound and free ligand. Binding affinities were calculated by non-linear curve fitting (Prism, Graphpad) (Table A.2) using the following equation:

$$
Y=\left[\left(B_{\max }\right)(X)\right] /\left[X+K_{d}\right]
$$

where $Y$ is the fraction of total bound ligand, $B_{\max }$ is the maximum concentration of ligand binding sites, $X$ is the concentration of labeled ligand, and $K_{d}$ is the equilibrium dissociation constant of the labeled ligand.

\section{BCL-xL Crosslinking}

Structure-based crosslinking of BCL-xL $\Delta C$ E7C and BCL-xL $\Delta C$ N175C cysteine mutants was performed based on the previously described experiment ( $\mathrm{O}^{\prime} \mathrm{Neill}$ et al., 2006) with minor modifications. Briefly, the indicated concentrations of the BCL-xL $\Delta C$ E7C and BCL-xL $\Delta C$ N175C were mixed at equimolar concentrations and incubated with or without a 5-fold molar excess of the irreversible, sulfhydryl crosslinking agent bismaleimidohexane (BMH) (Thermo Scientific) in the presence or absence of the indicated concentration of PUMA ${ }^{\mathrm{BH} 3}$. SDS-PAGE followed by western blot analysis for $\mathrm{BCL}-\mathrm{xL}$ was used to determine the oligomeric status of BCL-xL for each treatment. Treatment of BCL-xL $\Delta \mathrm{C}$ or the two BCL-xL $\Delta \mathrm{C}$ cysteine mutants individually or mixed as described above with buffer alone served as negative controls.

\section{Disulfide Bond Introduction}

Creation of a BCL-xL $\Delta \mathrm{C}$ double cysteine mutant construct that would form a disulfide bond and "trap" the protein in its monomeric form without disrupting the BH3 domain binding groove was guided using the software Disulfide By Design (Dombkowski, 2003). A BCL-xL $\Delta \mathrm{C}$ double cysteine mutant was generated and screened for successful disulfide bond formation using a free thiol assay as described (Pham et al., 2007).

\section{Electrophoresis, Immunoblotting, and Co-immunoprecipitation}

SDS-PAGE was conducted using the Criterion XT 4-12\% gel system (BioRad) with $1 \mathrm{X}$ MOPS buffer at $150 \mathrm{~V}$. Native PAGE was performed on $18 \%$ Tris-HCl polyacrylamide gels (BioRad) in native running buffer $(25 \mathrm{mM}$ Tris $\mathrm{pH} 8.3$, $192 \mathrm{mM}$ glycine), and samples were prepared using the Native PAGE Sample Loading Kit (Invitrogen). For western blot analysis, proteins were transferred to nitrocellulose by 
standard conditions, blocked in 5\% milk/Tris Buffered Saline Tween-20 (TBST) and primary antibodies (in blocking buffer: BCL-xL (SantaCruz Biotechnology, clone S-18) 1:1,000; cytochrome c (Pharmingen, clone 7H8.2C12) 1:1,000; p53 (SantaCruz Biotechnology, clone D07) 1:1,000; PUMA (Cell Signaling or Sigma) 1:1,000 and FLAG (SantaCruz Biotechnology, clone M2) 1:1,000 were incubated overnight at $4{ }^{\circ} \mathrm{C}$. The secondary antibody $\left(1: 5,000\right.$ in blocking buffer) was incubated at $25^{\circ} \mathrm{C}$ for $1 \mathrm{hr}$ before standard enhanced chemiluminescence detection. Blocking buffer consisted of $5.0 \%$ lowfat milk in TBST.

BCL-xL $\Delta \mathrm{C} \cdot \mathrm{p} 53$ complexes were produced for co-immunoprecipitation by combining $10 \mathrm{nM}$ recombinant BCL-xL $\Delta \mathrm{C}$ with purified baculovirus-expressed $\mathrm{p} 53$ (ProteinOne) or p53 ${ }^{\mathrm{UVIP}}$ in HE buffer (10 mM HEPES, pH 7.4, 1 mM EDTA) overnight at $4{ }^{\circ} \mathrm{C}$ (Chipuk et al., 2005b; Chipuk et al., 2004). Anti-BCL-xL (clone H-5) or anti-p53 (clone DO7) antibodies and protein A/G-agarose beads (Thermo Scientific) were added, incubated overnight at $4{ }^{\circ} \mathrm{C}$, and washed 3 times to remove any unbound material. Protein A/G-agarose beads were boiled in 1X SDS-PAGE loading dye, and precipitated proteins were visualized by standard SDS-PAGE and western blot techniques.

FLAG-PUMA co-immunoprecipitation experiments were performed by Dr. Jerry Chipuk in the laboratory of Dr. Douglas Green (Department of Immunology, St. Jude). For FLAG-PUMA co-immunoprecipitations, cells were lysed in 1\% CHAPS, $150 \mathrm{mM}$ $\mathrm{NaCl}, 5 \mathrm{mM} \mathrm{MgCl} 2,1 \mathrm{mM}$ EDTA and 10\% glycerol. Lysates $(100 \mu \mathrm{g})$ were combined with $1 \mu \mathrm{g}$ FLAG M2 antibody, $25 \mu 150 \%$ slurry of Protein A/G Plus Agarose (Santa Cruz Biotechnology) and incubated end over end for 2 hours at $4{ }^{\circ} \mathrm{C}$. Agarose beads were washed 3 times in lysis buffer, eluted with $100 \mu \mathrm{g} / \mathrm{ml} \mathrm{FLAG} \mathrm{peptide,} \mathrm{and} \mathrm{precipitated}$ proteins were visualized by standard SDS-PAGE and western blot techniques.

\section{Heavy Membrane Preparation and Cytochrome c Release}

Heavy membrane preparation and cytochrome c release assays were performed by Dr. Jerry Chipuk in the laboratory of Dr. Douglas Green (Department of Immunology, St. Jude). Heavy membrane fractions (referred to as mitochondria) were purified from murine liver, usually female, under 3 months, using dounce homogenization and differential centrifugation in mitochondrial isolation buffer (MIB: $200 \mathrm{mM}$ mannitol, 68 mM sucrose, 10 mM HEPES-KOH pH 7.4, 10 mM KCl, 1 mM EDTA, 1 mM EGTA, 0.1 $\%$ BSA).

For MOMP assays, mitochondria were incubated in MIB supplemented to 110 $\mathrm{mM} \mathrm{KCl}$ (mitochondrial assay buffer, MAB), plus or minus proteins and peptides (final concentrations and order of addition are indicated in the text and figure legends below) for 60 minutes at $37^{\circ} \mathrm{C}$. Reactions were then fractionated into supernatant and pellet by centrifugation at 5,500 x g for $5 \mathrm{~min}$, and analyzed by SDS-PAGE and western blot with an anti-cytochrome c antibody. 
For MOMP reconstitution studies wherein $b a k^{-/-} b a x^{-/}$mitochondria were used, heavy membrane fractions were isolated from the livers of poly(deoxyinosinicdeoxycytidylic) acid sodium salt (poly-dIdC) treated MxCre bak ${ }^{-/-}$bax ${ }^{f /-}$ mice. Three separate injections (each separated by one day) of poly-dIdC allowed interferon stimulation, especially in the liver. Induced interferon promoted activation of the $M x$ promoter, driving expression of Cre recombinase and subsequent removal of the remaining $\mathrm{MxCre}$ floxed bax allele.

For concurrent binding and MOMP assays, $10 \mathrm{nM}$ BCL-xL $\Delta \mathrm{C} \cdot \mathrm{p} 53^{\mathrm{UVIP}}$ complexes were produced and captured by combining recombinant $\mathrm{BCL}-\mathrm{xL} \Delta \mathrm{C}, \mathrm{p} 53^{\mathrm{UVIP}}$ and $\mathrm{Ni}^{+2}$ NTA agarose in PBS at $25^{\circ} \mathrm{C}$ for 2 hrs before pelleting $\left(1,000 \mathrm{x} \mathrm{g}, 5\right.$ minutes at $\left.25^{\circ} \mathrm{C}\right)$ and washing the complex three times with PBS. The final pellet was resuspended in MAB (without EDTA or EGTA), indicated peptides were added along with BAX and mitochondria, and incubated for $1 \mathrm{hr}$ at $37^{\circ} \mathrm{C}$ before fractionation and western blot analysis for cytochrome $\mathrm{c}, \mathrm{BCL}-\mathrm{xL} \Delta \mathrm{C}$ and $\mathrm{p} 53^{\mathrm{UVIP}}$.

\section{Cell Culture, Transfection, and Survival Assays}

Cell culture, transfection, and survival assays were performed by Dr. Jerry Chipuk in the laboratory of Dr. Douglas Green (Department of Immunology, St. Jude). Mouse embryonic fibroblasts (MEFs; SV40 immortalized wild type, $p 53^{---}$and $p u m a^{-/-}$) were cultured in DMEM containing 10\% FBS, 100 mM 2-mercaptoethanol, $2 \mathrm{mM}$ Lglutamine and antibiotics. The MEF panel was routinely replaced to minimize cultureinduced accumulation of direct activator proteins. Cells were transfected using Lipofectamine 2000 according to the manufacturer's instructions for 6 hours under serum-free conditions (pCMV5 and pCMVneoBam-FLAG-PUMA wild type or W71A). Green fluorescent protein (pUS9-GFP) was co-transfected as an efficiency marker (30$50 \%$ of cells were usually transfected). The next day, cells were treated with indicated doses of TNF/CHX or UV for $6 \mathrm{~h}$ and $24 \mathrm{~h}$, respectively; before trypsinization, RIPA lysis, immunoprecipitation and/or AnnexinV-PE labeling and analysis by flow cytometry.

\section{Microinjection and Confocal Microscopy}

Microinjection and confocal microscopy experiments were performed by Dr. Jerry Chipuk in the laboratory of Dr. Douglas Green (Department of Immunology, St. Jude). MCF-7 SMAC-GFP cells (Munoz-Pinedo et al., 2006) were plated on glass bottom dishes (MatTek) the day before injection in DMEM (containing: $200 \mathrm{mM}$ L-glutamine, $10 \%$ heat inactivated FBS and antibiotics) and incubated at $37^{\circ} \mathrm{C}$ with $5 \% \mathrm{CO}_{2}$. At the time of injection, cells were approximately $90 \%$ confluent and treated with $20 \mu \mathrm{M}$ QVD-OPh (R\&D Systems) (except for TNF/CHX treated cells). Cells were co-injected with Texas Red dextran $(0.2 \mu \mathrm{g} / \mu \mathrm{l}, 10 \mathrm{kDa}$, Invitrogen, Molecular Probes $)$ in HE buffer (10 mM HEPES pH 7.4, $1 \mathrm{mM}$ EDTA); the needle concentrations were: $5.5 \mu \mathrm{M}$ C8-BID, BCL-xL $\Delta \mathrm{C}$ monomer $(50 \mu \mathrm{M})$, and BCL-xL $\Delta \mathrm{C}$ apo-dimer $(25 \mu \mathrm{M})$. Approximately 1050 femtoliters were injected per cell; the uncertainty in injection volume was due to the 
back pressure within the cell, which was dictated by where in the cytoplasm the needle entered. Cells were injected using an Eppendorf InjectMan NI2/FemtoJet microinjection system (Brinkmann Instruments), mounted on a Nikon TE2000-U inverted microscope (Nikon USA), with an ELDW 40X Plan Fluor phase-contrast objective (NA 0.6). Cells were kept in the stage for less than $20 \mathrm{~min}$ at a time. Injection parameters: Pi: $30-120$ $\mathrm{hPa}, \mathrm{Ti}: 0.5 \mathrm{~s}, \mathrm{Pc}: 20 \mathrm{hPa}$. Immediately after injection cells were allowed to recover at 37 ${ }^{\circ} \mathrm{C}, 5 \% \mathrm{CO}_{2}$. Confocal microscopy of live cells was performed with a Marianas spinning disk confocal imaging system (Intelligent Imaging Innovations/3i) consisting of a CSU22 confocal head (Yokogowa Electric Corporation), DPSS lasers (CrystaLaser) with wavelengths of $445 \mathrm{~nm}, 473 \mathrm{~nm}, 523 \mathrm{~nm}, 561 \mathrm{~nm}$, and $658 \mathrm{~nm}$, and a Carl Zeiss 200M motorized inverted microscope (Carl Zeiss MicroImaging), equipped with spherical aberration correction optics (3i). Temperature was maintained at $37{ }^{\circ} \mathrm{C}$ and $5 \% \mathrm{CO}_{2}$ using an environmental control chamber (Solent Scientific). Images were acquired with a Zeiss Plan-Neofluar 40x 1.3 NA DIC objective on a CascadeII 512 EMCCD (Photometrics), using SlideBook 4.2 software (3i) after indicated treatments. Greater than 200 injected cells were analyzed for each condition.

\section{Protein Concentration Determination}

The concentrations of all prepared recombinant proteins were determined by UV absorbance at $280 \mathrm{~nm}$ in a denaturing buffer containing $20 \mathrm{mM}$ phosphate, $\mathrm{pH} 6.5,6.0 \mathrm{M}$ guanidine hydrochloride (Bairoch et al., 2005; Gill and von Hippel, 1989). The molar extinction coefficients were determined using the ProteinParameters tool (ExPASy server; https://us.expasy.org/tools/protparam.html). 


\section{CHAPTER 3. RESULTS}

\section{PUMA Is an Intrinsically Disordered Protein that Induces BCL-xL Dimerization}

We generated purified recombinant, full-length human PUMA beta (hereafter referred to as PUMA) and examined its structural features. From CD and NMR spectroscopy analyses (Figure 3.1), we determined that full-length PUMA protein is an intrinsically disordered protein (IDP), which is similar to other BH3-only proteins (Hinds et al., 2006). The CD spectrum of PUMA exhibited the features of an extensively disordered protein, with a minimum value of molar ellipticity at $200 \mathrm{~nm}$ and no features indicative of highly populated secondary structure (Figure 3.1A). A weak feature at 222 $\mathrm{nm}$ is indicative of $\alpha$-helical secondary structure and is accounted for by the nascent helical structure within the $\mathrm{BH} 3$ domain by comparison of CD spectra from PUMA and the PUMA ${ }^{\mathrm{BH} 3}$ as well as amino and carboxyl terminal fragments of PUMA lacking the BH3 domain $\left[\mathrm{PUMA}^{\mathrm{N}}\right.$ and PUMA ${ }^{\mathrm{C}}$ (residues 1-67 and 93-131, respectively); Figure 3.1A]. Further, the $2 \mathrm{D}^{1} \mathrm{H}^{-15} \mathrm{~N}$ heteronuclear single-quantum coherence (HSQC) NMR spectrum of ${ }^{15} \mathrm{~N}$-labeled PUMA exhibited limited ${ }^{1} \mathrm{H}$ chemical shift dispersion $(\leq 1 \mathrm{ppm})$ (Figure 3.1B), which is characteristic of IDPs (Kriwacki et al., 1996). The protein exhibited the appropriate number of peaks ( $\sim 15$ Gly residues), indicating it was predominantly a single, homogenous species.

Previous biochemical studies suggested that the PUMA BH3 domain is the principal site of interaction with BCL-xL (Chen et al., 2005; Chipuk et al., 2005b; Chipuk et al., 2008; Kuwana et al., 2005). We confirmed this by comparing the affinities of a PUMA BH3 domain peptide (PUMA ${ }^{\mathrm{BH} 3}$ ) and PUMA protein for binding BCL-xL $\Delta \mathrm{C}$ using isothermal titration calorimetry (ITC) (Figure 3.2). These results showed that both full-length PUMA and PUMA ${ }^{\mathrm{BH} 3}$ bound BCL-xL equivalently.

To further analyze binding of PUMA ${ }^{\mathrm{BH} 3}$ to $\mathrm{BCL}-\mathrm{xL} \Delta \mathrm{C}$, sedimentation velocity analytical ultracentrifugation (AUC) experiments were performed. A functional $\mathrm{BCL}-\mathrm{xL}$ protein lacking the unstructured loop as well as the C-terminal 22 residues, hereafter referred to as BCL-xL $\Delta \mathrm{L} \Delta \mathrm{C}$ (Muchmore et al., 1996b), was used for AUC experiments. While monomeric BCL-xL $\Delta \mathrm{L} \Delta \mathrm{C}$ and the BCL-xL $\Delta \mathrm{L} \Delta \mathrm{C} \cdot \mathrm{BIM}^{\mathrm{BH} 3}$ complex (which exhibits 1:1 stoichiometry) (Liu et al., 2003), sedimented with $s$ values corresponding to the expected masses (monomeric BCL-xL $\Delta \mathrm{L} \Delta \mathrm{C}$ : theoretical mass $=20.8 \mathrm{kDa}$, observed mass $=20.4 \mathrm{kDa}$; BCL-xL $\Delta \mathrm{L} \Delta \mathrm{C} \cdot \mathrm{BIM}^{\mathrm{BH} 3}$ : theoretical mass $=23.9 \mathrm{kDa}$, observed mass $=$ $23.9 \mathrm{kDa}$ ), the BCL-xL $\Delta \mathrm{L} \Delta \mathrm{C} \cdot \mathrm{PUMA}{ }^{\mathrm{BH} 3}$ complex sedimented with an $s$ value consistent with a $2: 2$ heterodimeric species $\left(2: 2 \mathrm{BCL}-\mathrm{xL} \Delta \mathrm{L} \Delta \mathrm{C} \cdot \mathrm{PUMA}^{\mathrm{BH} 3}\right.$ : theoretical mass $=47.6$ $\mathrm{kDa}$, observed mass $=46.7 \mathrm{kDa}$ ) (Figure 3.3). Sedimentation equilibrium AUC experiments yielded molecular weight values for BCL-xL $\Delta \mathrm{L} \Delta \mathrm{C}$, the BCL$\mathrm{xL} \Delta \mathrm{L} \Delta \mathrm{C} \cdot \mathrm{BIM}^{\mathrm{BH} 3}$ complex, and the BCL-xL $\Delta \mathrm{L} \Delta \mathrm{C} \cdot \mathrm{PUMA}^{\mathrm{BH} 3}$ complex that were in agreement with the sedimentation velocity AUC data. Collectively, these AUC data strongly suggested that addition of PUMA ${ }^{\mathrm{BH} 3}$-induced $\mathrm{BCL}-\mathrm{xL} \Delta \mathrm{L} \Delta \mathrm{C}$ dimerization. BCL$\mathrm{xL}$ has previously been shown to dimerize via swapping of the protein's $\mathrm{C}$ - terminal 22 residues (Jeong et al., 2004), and a crystal structure of a domain-swapped BCL-xL $\Delta \mathrm{C}$ 

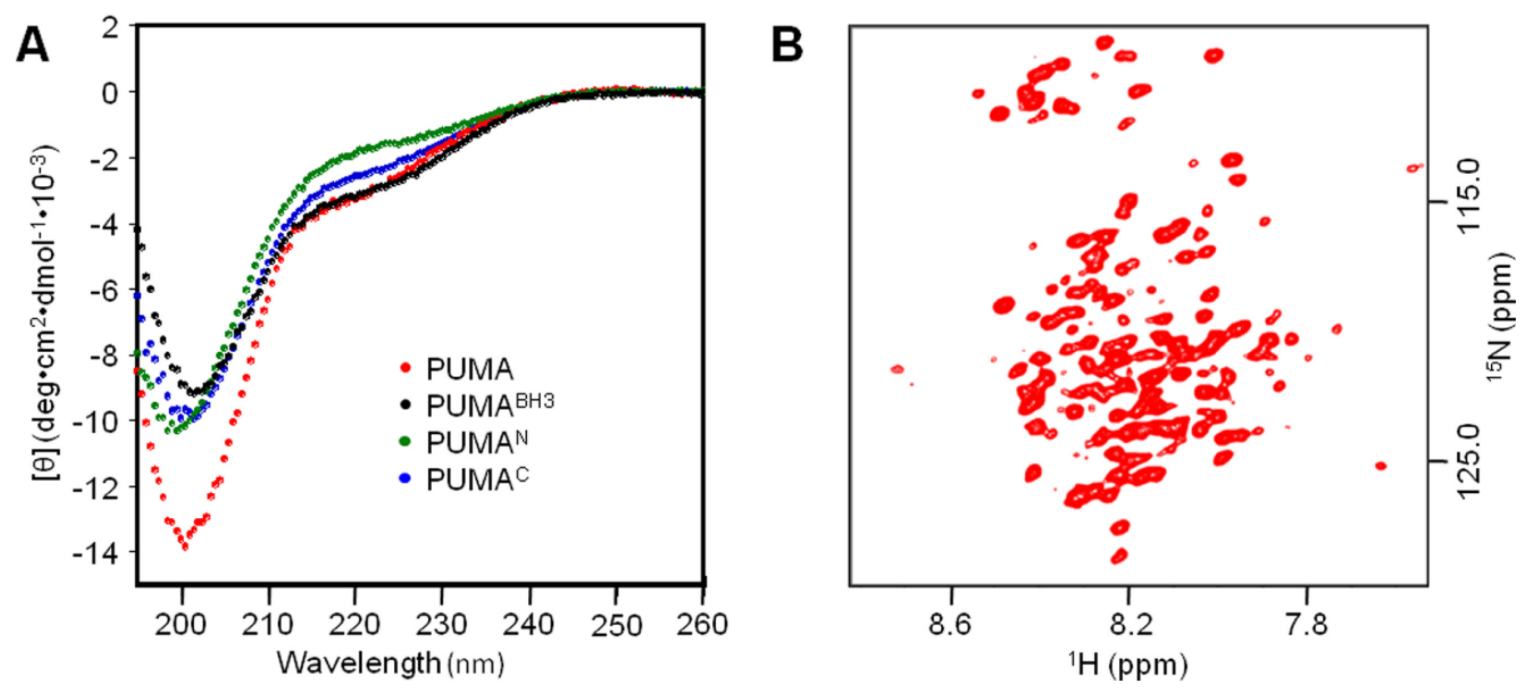

Figure 3.1 PUMA Is an Intrinsically Disordered Protein.

(A) The circular dichroism (CD) spectrum of PUMA exhibited a minimum value of molar ellipticity at $200 \mathrm{~nm}$, consistent with a lack of highly populated secondary structure. A weak feature at $222 \mathrm{~nm}$ indicated alpha-helical secondary structure that can be attributed to nascent helical structure within the PUMA BH3 domain by comparison of CD spectra for PUMA with spectra for peptides corresponding to the PUMA BH3 domain and the amino and carboxyl terminal fragments of PUMA lacking the $\mathrm{BH} 3$ domain $\left(\mathrm{PUMA}^{\mathrm{BH} 3}, \mathrm{PUMA}^{\mathrm{N}}\right.$, and PUMA ${ }^{\mathrm{C}}$, respectively). (B) The $2 \mathrm{D}{ }^{1} \mathrm{H}^{15}{ }^{15} \mathrm{NSQC}$ spectrum of PUMA exhibited the appropriate number of resonances. PUMA resonances were dispersed over a narrow ${ }^{1} \mathrm{H}$ chemical shift range $(\leq 1 \mathrm{ppm})$ indicating that PUMA is intrinsically disordered. 
A

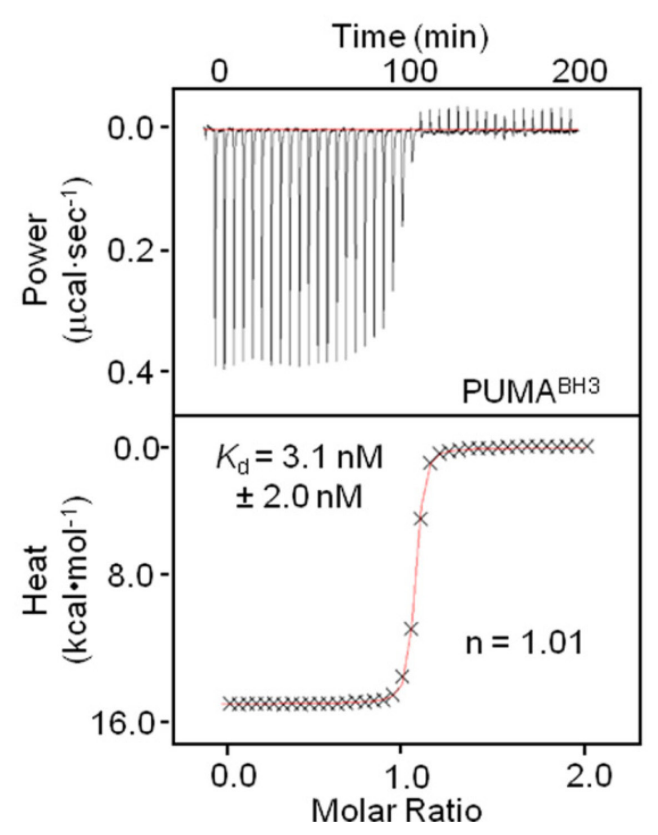

B

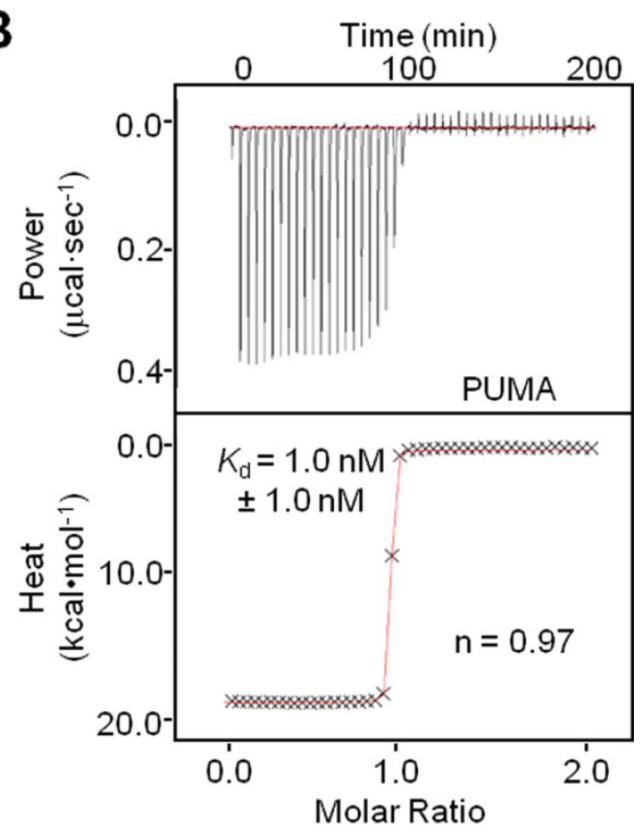

Figure 3.2 The BH3 Domain of PUMA Is Sufficient for Binding BCL-xL.

ITC binding isotherms recorded for BCL-xL $\Delta$ C titrated into a solution of $\mathrm{PUMA}^{\mathrm{BH} 3}$ (A) or PUMA (B). Errors represent the standard deviation calculated from at least two independent experiments. 

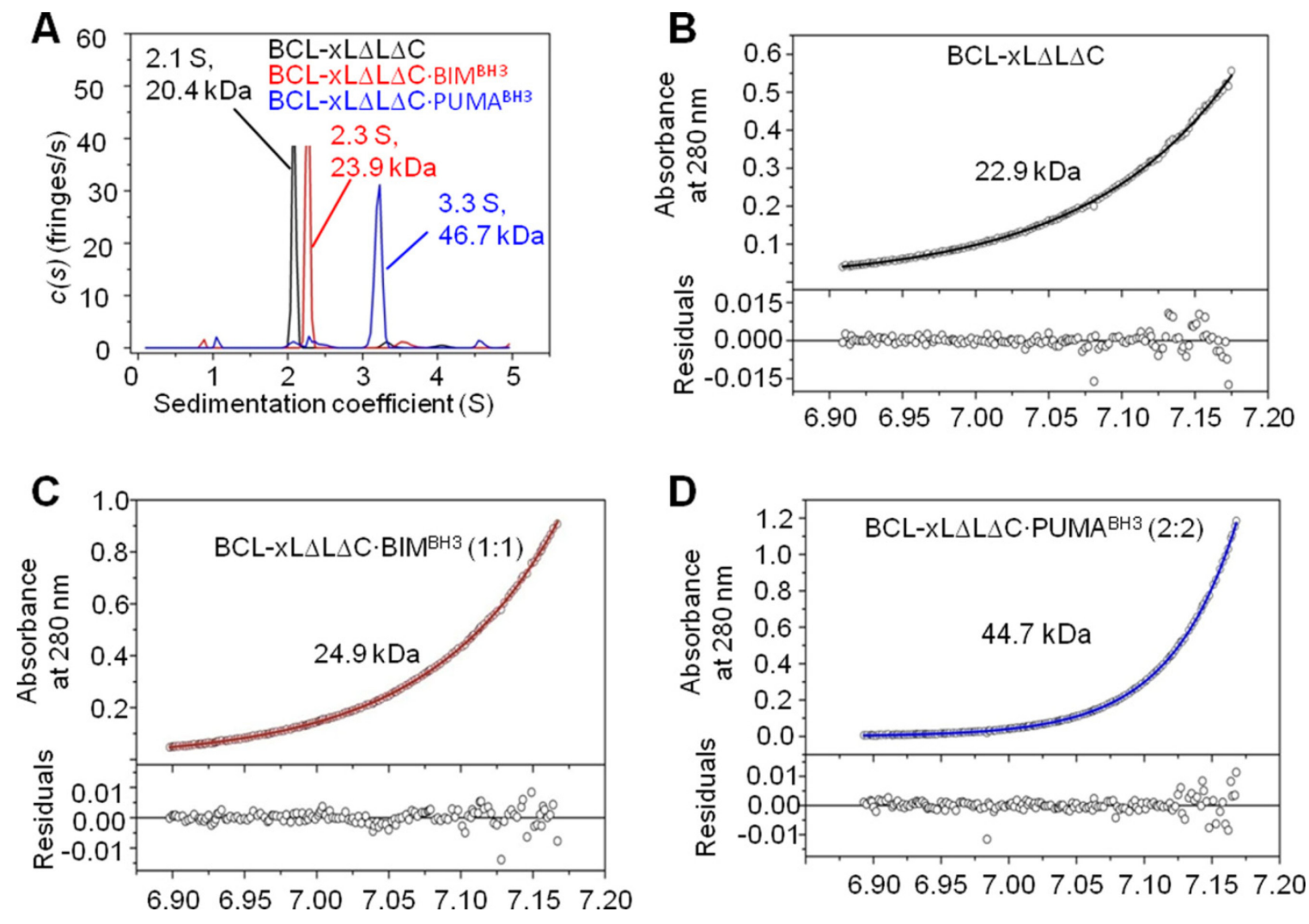

Figure 3.3 Analytical Ultracentrifugation of Indicated BCL-xL $\Delta C$ Species. (A) The sedimentation velocity profiles, $s$ values, and experimental molecular masses for $\mathrm{BCL}-\mathrm{xL} \Delta \mathrm{L} \Delta \mathrm{C}$ (black), BCL-xL $\Delta \mathrm{L} \Delta \mathrm{C} \cdot \mathrm{BIM}^{\mathrm{BH} 3}$ (red), and BCL-xL $\Delta \mathrm{L} \Delta \mathrm{C} \cdot \mathrm{PUMA}^{\mathrm{BH} 3}$ (blue). Sedimentation equilibrium AUC analysis of monomeric BCL-xL $\Delta L \Delta C, B C L-$ $\mathrm{xL} \Delta \mathrm{L} \Delta \mathrm{C} \cdot \mathrm{BIM}^{\mathrm{BH} 3}$ domain peptide and BCL-xL $\Delta \mathrm{L} \Delta \mathrm{C} \cdot \mathrm{PUMA}^{\mathrm{BH} 3}$ domain peptide. (B-D) Solid lines represent the global nonlinear least squares best-fit to a single species model with a molar mass of $22.9 \mathrm{kDa}$ for BCL-xL $\Delta \mathrm{L} \Delta \mathrm{C}$ (black), $24.9 \mathrm{kDa}$ for BCL$\mathrm{xL} \Delta \mathrm{L} \Delta \mathrm{C} \cdot \mathrm{BIM}^{\mathrm{BH} 3}(1: 1)$ (red) and $44.7 \mathrm{kDa}$ for BCL-xL $\Delta \mathrm{L} \Delta \mathrm{C} \cdot \mathrm{PUMA}^{\mathrm{BH}}{ }^{2}$ (2:2) (blue). The RMSD for the respective fits were between 0.0023 and 0.0030 absorbance units. For clarity only the samples with respective loading protein concentrations of 4.6, 5.6 and 2.6 $\mu \mathrm{M}$ and centrifuged at the rotor speed 28,000 rpm are shown. 
apo-dimer (induced via purification of the protein under extreme alkaline conditions) was previously reported (O'Neill et al., 2006).

To investigate the phenomenon of PUMA ${ }^{\mathrm{BH} 3}$-induced BCL-xL dimerization, we established a native polyacrylamide gel electrophoresis (PAGE) gel system to resolve apo (i.e., ligand-free) monomeric BCL-xL $\Delta \mathrm{C}$ and the $\mathrm{BCL}-\mathrm{xL} \Delta \mathrm{C} \cdot \mathrm{PUMA}^{\mathrm{BH}}{ }^{\mathrm{B}}$ dimer. A stable, unbound, "BCL-xL $\Delta C$ apo-dimer" was prepared by heat treatment, as described (Denisov et al., 2007b); BCL-xL $\Delta \mathrm{C}$ monomer and BCL-xL $\Delta \mathrm{C}$ apo-dimer were used as standards in our native PAGE assays.

Consistent with the AUC results described above, the addition of $\mathrm{PUMA}^{\mathrm{BH} 3}$ to BCL-xL $\Delta \mathrm{C}$ monomer resulted in dose-dependent, stoichiometric formation of the dimeric BCL-xL $\Delta C \cdot P U M A^{\mathrm{BH} 3}$ complex (Figure 3.4A). BCL-xL $\Delta C$ G138A, a mutant form of BCL-xL that does not bind to pro-apoptotic effector BCL-2 proteins (i.e., BAK or $\mathrm{BAX}$ ) or BH3-only proteins (Yang et al., 1995), was used as a negative control in the native PAGE assay. As expected, the addition of PUMA ${ }^{\mathrm{BH} 3}$ to BCL-xL $\Delta \mathrm{C}$ G138A did not cause dimerization, whereas dimerization was observed with wild type BCL-xL $\Delta C$ (Figure 3.4B).

To determine if BCL-xL $\Delta \mathrm{C}$ dimerization was specific to the PUMA BH3 domain, seven additional BH3 domain peptides (Table 3.1) were evaluated for inducing BCL$\mathrm{xL} \Delta \mathrm{C}$ dimerization using the native PAGE assay. As expected, the addition of a two-fold molar excess of PUMA ${ }^{\mathrm{BH} 3}$ to $\mathrm{BCL}-\mathrm{xL} \Delta \mathrm{C}$ monomer resulted in complete BCL-xL $\Delta \mathrm{C}$ dimerization, whereas the same concentration of $\mathrm{BH} 3$ domain peptides from $\mathrm{BAD}, \mathrm{BAK}$, BAX, BID, BIM, HRK and Noxa failed to induce BCL-xL $\Delta C$ dimerization (Figure 3.4C). ITC analyses confirmed that all but one of the $\mathrm{BH} 3$ domain peptides used in this assay were capable of binding to BCL-xL $\Delta \mathrm{C}$ (Figure 3.5). As a negative control, the $\mathrm{Noxa}^{\mathrm{BH} 3}$ did not bind to $\mathrm{BCL}-\mathrm{xL} \Delta \mathrm{C}$ due to a restricted binding pattern for anti-apoptotic proteins (Noxa ${ }^{\mathrm{BH} 3}$ only binds BFL-1 and MCL-1 (Chen et al., 2005)). All thermodynamic parameters were calculated (Table A.4)

The ability of the PUMA ${ }^{\mathrm{BH} 3}$ to induce dimerization of other anti-apoptotic BCL-2 proteins (e.g., BCL-2, BFL-1 and MCL-1) was also evaluated. The addition of a two-fold molar excess of PUMA ${ }^{\mathrm{BH} 3}$ to BCL-2 $\Delta \mathrm{C}$, BFL-1 $\Delta \mathrm{C}$ or MCL-1 $\Delta \mathrm{C}$ did not result in the formation of higher-order, peptide-bound species (Figure 3.4D), which is consistent with recently reported structures of the BFL-1 $\triangle \mathrm{C} \cdot \mathrm{PUMA}^{\mathrm{BH}}{ }^{\mathrm{B}}$ (Smits et al., 2008) and MCL$1 \Delta C \cdot \mathrm{PUMA}^{\mathrm{BH} 3}$ (Day et al., 2008) complexes. These results suggested that $\mathrm{PUMA}^{\mathrm{BH} 3}$ induced dimerization of the anti-apoptotic BCL-2 family member BCL-xL was unique to the BCL-xL·PUMA interaction.

\section{PUMA-induced BCL-xL Dimer Is Not in Dynamic Equilibrium}

We then examined the stability of the PUMA-induced BCL-xL dimer by several approaches. PUMA ${ }^{\mathrm{BH} 3}$ plus BCL-xL $\Delta \mathrm{C}$ was resolved by analytical size exclusion

chromatography, collected, and the diluted material was subsequently rerun. In both cases 


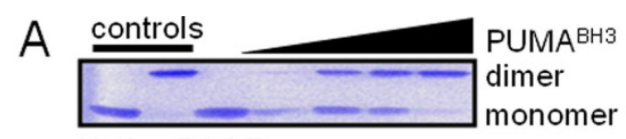

Native PAGE

BCL-xL $\triangle \mathrm{C}: 50 \mu \mathrm{M}$

PUMA ${ }^{B H 3}: 0,0.25,0.5,0.75,1$ molar equivalents
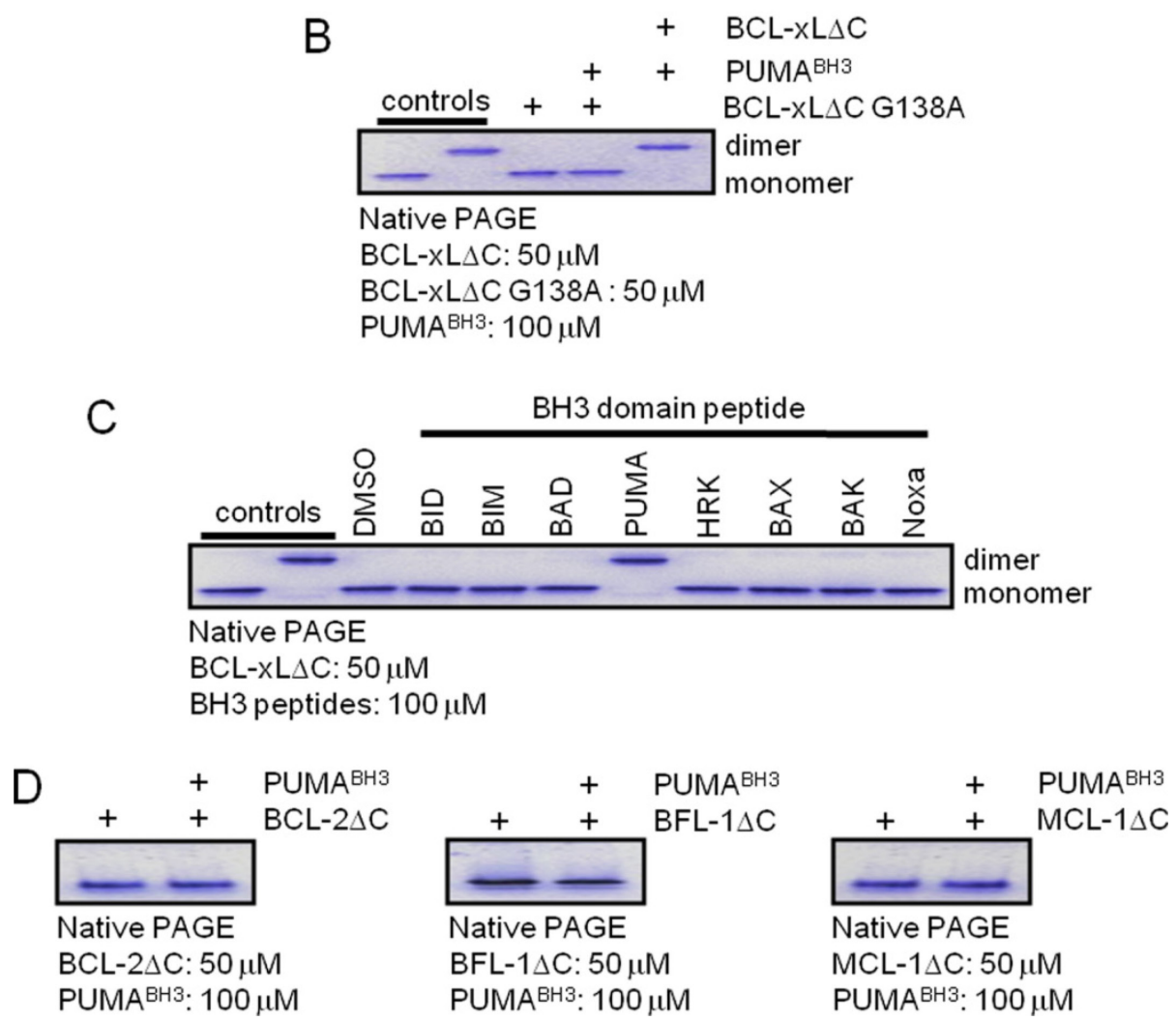

\section{Figure 3.4 Native PAGE Analysis of PUMA-Anti-apoptotic BCL-2 Family Protein Complexes.}

(A)The addition of PUMA ${ }^{\mathrm{BH} 3}$ to BCL-xL $\triangle \mathrm{C}$ monomer caused the stoichiometric conversion of BCL-xL $\Delta \mathrm{C}$ to a dimeric species. BCL-xL $\Delta \mathrm{C}(50 \mu \mathrm{M})$ was treated with $\operatorname{PUMA}^{\mathrm{BH} 3}\left(0,0.25,0.5,0.75\right.$ and 1.0 molar equivalents) for one hour at $37^{\circ} \mathrm{C}$ and subjected to native PAGE followed by Coomassie staining. BCL-xL $\Delta \mathrm{C}$ monomer and $\mathrm{BCL}-\mathrm{xL} \Delta \mathrm{C}$ apo-dimer were used as native PAGE controls. (B) $\mathrm{PUMA}^{\mathrm{BH} 3}$-induced dimerization of BCL-xL $\Delta \mathrm{C}$ required $\mathrm{G} 138$ within the $\mathrm{BCL}-\mathrm{xL} \Delta \mathrm{C}$ canonical $\mathrm{BH} 3$ domain-binding groove. The BCL-xL $\Delta \mathrm{C} \mathrm{G138A}$ mutant $(50 \mu \mathrm{M})$ was treated with PUMA $^{\mathrm{BH} 3}(100 \mu \mathrm{M})$ for one hour at $37^{\circ} \mathrm{C}$ and subjected to native PAGE followed by Coomassie staining. (C) BCL-xL $\Delta \mathrm{C}(50 \mu \mathrm{M})$ was treated with the indicated BH3 domain peptides $(100 \mu \mathrm{M})$ for one hour at $37^{\circ} \mathrm{C}$ and subjected to native PAGE followed by Coomassie staining. Noxa ${ }^{\mathrm{BH} 3}$ was used as a negative control. (D) $\mathrm{PUMA}^{\mathrm{BH} 3}$ did not induce the dimerization of other anti-apoptotic BCL-2 proteins. BCL-2 $\Delta \mathrm{C}, \mathrm{BFL}-1 \Delta \mathrm{C}$ or MCL-1 $\Delta \mathrm{C}(50 \mu \mathrm{M})$ was treated with PUMA ${ }^{\mathrm{BH} 3}(100 \mu \mathrm{M})$ for one $\mathrm{hr}$ at $37^{\circ} \mathrm{C}$ and subjected to native PAGE followed by Coomassie staining. 
Table 3.1 BH3 Domain Peptide Sequences.

\begin{tabular}{cc}
\hline BH3 Domain Peptide & Peptide Sequence (N- to C-terminal) \\
\hline BAD & NLWAAQRYGRELRRMSDEFVDSFK \\
BAK & PSSTMGQVGRQLAIIGDDINR \\
BAX & PQDASTKKSECLKRIGDELDSNMEL \\
BID & QEDIIRNIARHLAQVGDSMDRSIPP \\
BIM & DMRPEIWIAQELRRIGDEFNAYYAR \\
HRK & RSSAAQLTAARLKALGDELHQRTMW \\
Noxa & PAELEVECATQLRRFGDKLNFRQKL \\
PUMA & EEQWAREIGAQLRRMADDLNAQYER \\
\hline
\end{tabular}



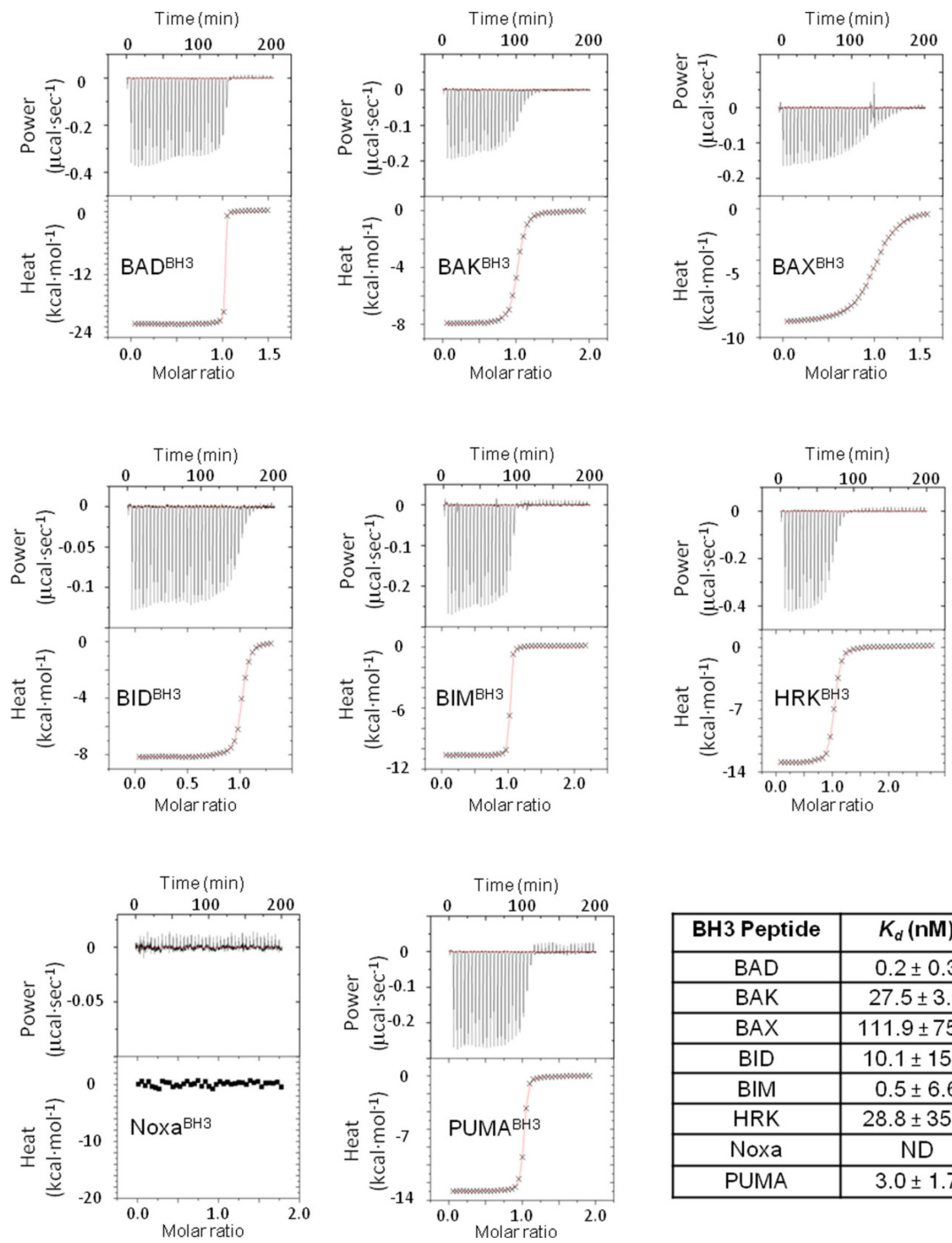

\begin{tabular}{|c|c|}
\hline BH3 Peptide & $K_{d}(\mathbf{n M})$ \\
\hline BAD & $0.2 \pm 0.3$ \\
\hline BAK & $27.5 \pm 3.0$ \\
\hline BAX & $111.9 \pm 75.0$ \\
\hline BID & $10.1 \pm 15.0$ \\
\hline BIM & $0.5 \pm 6.6$ \\
\hline HRK & $28.8 \pm 35.0$ \\
\hline Noxa & ND \\
\hline PUMA & $3.0 \pm 1.7$ \\
\hline
\end{tabular}

Figure 3.5 ITC Analysis of BH3 Domain Peptides Binding BCL-xL $\Delta C$.

(A) A $100 \mu \mathrm{M}$ solution of the indicated $\mathrm{BH} 3$ domain peptides was titrated from the ITC syringe into the ITC cell containing $10 \mu \mathrm{M}$ BCL-xL $\Delta \mathrm{C}$. Data were fit using a 1:1 binding model. Errors represent the standard deviation calculated from at least two independent experiments. 
BCL-xL $\Delta C$ resolved as a single peak with a molecular weight consistent with a 2:2 heterodimeric BCL-xL $\Delta C \cdot P U M A^{\mathrm{BH} 3}$ complex (Figure 3.6A). Similarly, sedimentation equilibrium $\mathrm{AUC}$ was again performed at different concentrations of $\mathrm{BCL}-\mathrm{xL} \Delta \mathrm{C}$ $\cdot \mathrm{PUMA}^{\mathrm{BH} 3}$, and in agreement with the previously obtained data from Figure 3.3D, results indicated formation of a stable dimer (Figure 3.6B). Finally, full-length PUMA and $\mathrm{BCL}-\mathrm{xL} \Delta \mathrm{C}$ were mixed together to form a 1:1 complex and subsequently resolved by native PAGE. Full-length PUMA was then competed from the complex using $\mathrm{BIM}^{\mathrm{BH} 3}$, which binds with affinity similar to PUMA (Figure 3.5) but does not induce dimer formation (Figure 3.4C). We found that under these conditions, the PUMA-induced dimer persisted even when one or both binding sites were effectively replaced with $\mathrm{BIM}^{\mathrm{BH} 3}$ (Figure 3.6C). Therefore, the BCL-xL $\Delta \mathrm{C}$ dimer induced by $\mathrm{PUMA}^{\mathrm{BH} 3}$ is stable, and was not detected to be in dynamic equilibrium with a monomeric complex.

\section{A Structure of BCL-xL $\Delta C$}

$\mathrm{X}$-ray crystallography was first used to confirm the oligomeric state of our recombinant human $\mathrm{BCL}-\mathrm{xL} \Delta \mathrm{C}$ prior to its co-crystallization with the PUMA ${ }^{\mathrm{BH} 3}$. BCL$\mathrm{xL} \Delta \mathrm{C}$ was crystallized in the same space group and unit cell as the published BCL-xL $\Delta \mathrm{C}$ structure (PDB code 1R2D) (Manion et al., 2004), and the structure was determined at $2.1 \AA$ resolution by direct refinement. As expected, BCL-xL $\Delta \mathrm{C}$ was found to be monomeric and virtually identical to $1 \mathrm{R} 2 \mathrm{D}$ with a $\mathrm{C} \alpha$ root mean square deviation (RMSD) of $0.5 \AA$ (Figure 3.7A). Also consistent with previous BCL-xL $\Delta \mathrm{C}$ structural studies, the protein comprises eight $\alpha$-helices $(\alpha 1-\alpha 8)$ and contains a 56 amino acid unstructured loop (residues 24-80) located between $\alpha 1$ and $\alpha 2$ for which there was no discernable electron density (Manion et al., 2004; Muchmore et al., 1996b).

\section{The Structure of the BCL-xL $\Delta C \cdot P U M A{ }^{\mathrm{BH} 3}$ Complex}

BCL-xL $\Delta \mathrm{C}$ was then co-crystallized with a 25 amino acid PUMA ${ }^{\mathrm{BH} 3}$ (Table 3.1), and the structure was determined to $2.9 \AA$ resolution by molecular replacement using our $2.1 \AA$ monomeric BCL-xL $\Delta \mathrm{C}$ structure as the search model. The asymmetric unit contained two monomers, molecules ' $\mathrm{A}$ ' and ' $\mathrm{B}$ ', but the electron density for molecule $\mathrm{A}$ was far superior. Density for the entire BCL-xL $\Delta \mathrm{C}$ molecule A was visible and readily interpretable apart from the unstructured loop, whereas density for molecule B was very poor for residues 101-121 and 132-133. Our structural conclusions on the complex are therefore largely based on molecule A. Clear electron density for the $\mathrm{PUMA}^{\mathrm{BH} 3}$ was visible in the initial $2 \mathrm{fo}-\mathrm{fc}$ map within the hydrophobic groove of molecule A formed by the $\mathrm{BH} 1, \mathrm{BH} 2$, and $\mathrm{BH} 3$ regions of $\mathrm{BCL}-\mathrm{xL} \Delta \mathrm{C}$. This region was previously shown to bind BH3 domain peptides (Petros et al., 2000; Sattler et al., 1997), and the density corresponded to amino acids 71-90 of the PUMA BH3 domain in which residues adopt an $\alpha$-helical conformation. The peptide binding groove of molecule $\mathrm{B}$ was not visible in the electron density map (residues 101-121) and it was not possible to discern whether $\mathrm{PUMA}^{\mathrm{BH} 3}$ was bound to this molecule. 
Figure 3.6 The PUMA-induced BCL-xL Dimer Shows No Signs of Dynamic Equilibrium.

(A) The PUMA ${ }^{\mathrm{BH} 3}$-induced BCL-xL dimer remained intact following injection of $20 \mu \mathrm{g}$ of the complex (cyan trace). The diluted complex was collected, re-injected, and still migrated as a single species with an identical retention volume as the initial run (red trace). BCL-xL monomer (diluted to match the absorbance of the re-injected PUMA ${ }^{\mathrm{BH}}$ induced BCL-xL dimer) was also run for comparison (blue trace). (B) Sedimentation equilibrium analysis of BCL-xL $\Delta C \cdot \mathrm{PUMA}^{\mathrm{BH} 3}$. Absorbance scans at $230 \mathrm{~nm}$ at equilibrium are plotted versus the distance from the axis of rotation. The protein was centrifuged in a $20 \mathrm{mM}$ sodium phosphate $\mathrm{pH} 7.0,100 \mathrm{mM} \mathrm{NaCl}$ buffer at $4{ }^{\circ} \mathrm{C}$ for at least $24 \mathrm{hr}$ at each rotor speed of 15, 22 and 28,000 rpm. The data fit best to a single species model. The solid lines represent the global nonlinear least squares best-fit of all the data sets to a single species model with a molecular weight of $50.1 \mathrm{kDa}$ (theoretical molecular weight $=53.3 \mathrm{kDa}$ ). For clarity only data for the smallest loading protein concentration of $0.19 \mu \mathrm{M}$ are shown. The RMSD for the fits were between 0.0013 0.0026 absorbance units. (C) BIM ${ }^{\mathrm{BH} 3}$ displacement of PUMA from PUMA-induced $\mathrm{BCL}-\mathrm{xL}$ dimer. Indicated concentrations of $\mathrm{BIM}^{\mathrm{BH} 3}$ were added to $\mathrm{BCL}-\mathrm{xL} \Delta \mathrm{C}$ or the PUMA-induced BCL-xL $\Delta \mathrm{C}$ dimer. Titrations were analyzed by native PAGE followed by western blot to detect BCL-xL oligomeric status. 

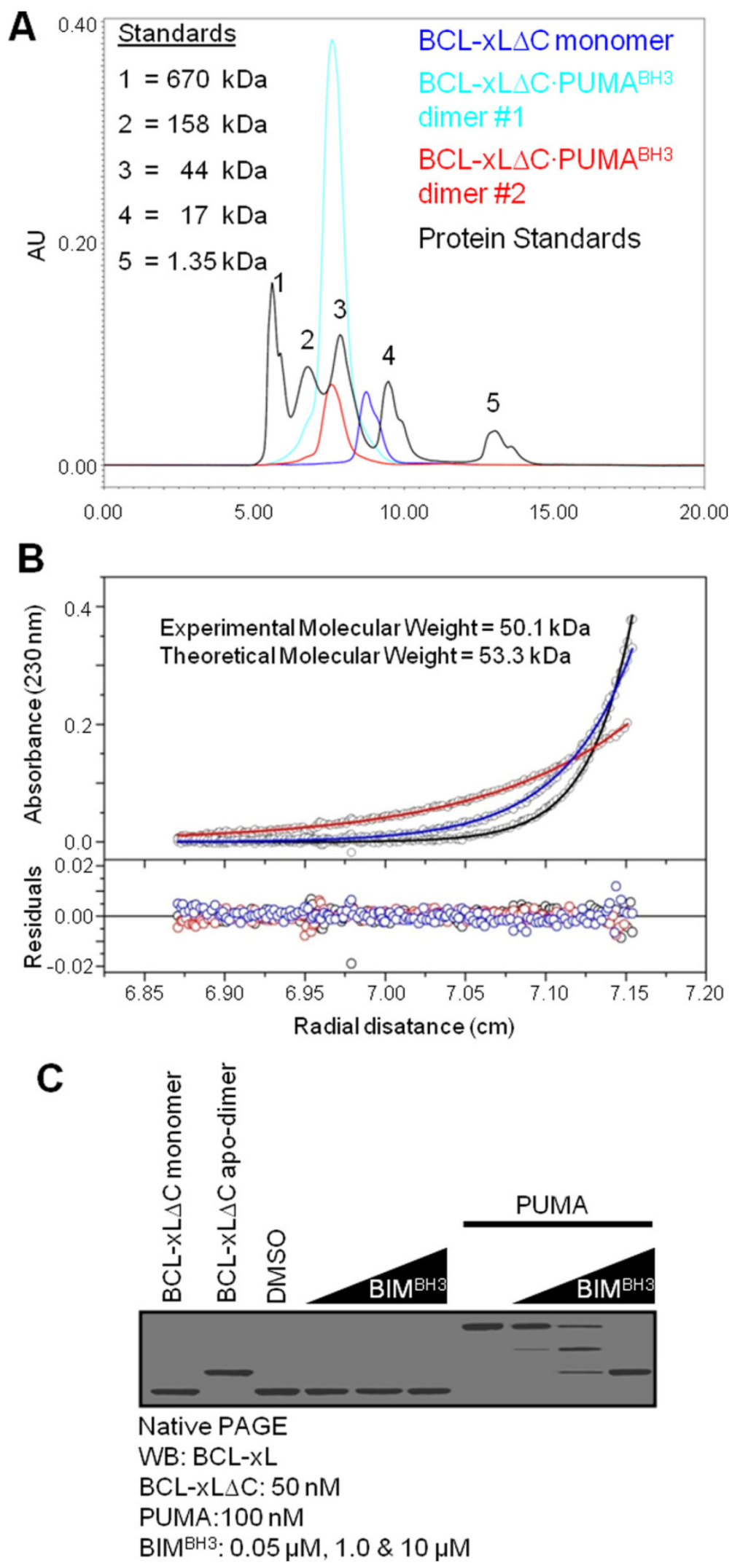

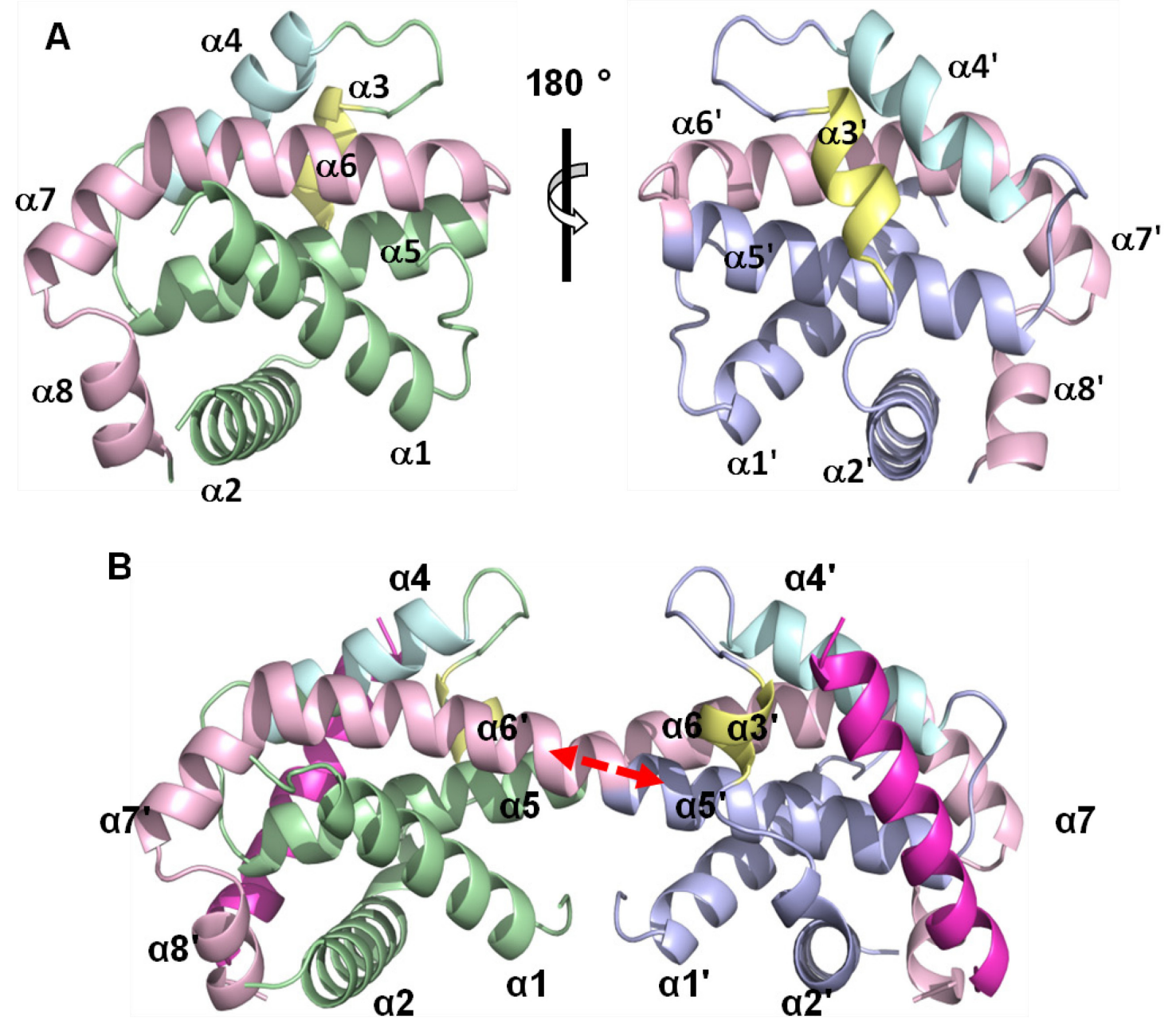

Figure 3.7 Structures of BCL-xL $\Delta C$ and the BCL-xL $\Delta C \cdot P U M A{ }^{B H 3}$ Complex. (A) The crystal structure of monomeric, human BCL-xL $\Delta \mathrm{C}$ solved at $2.1 \AA$ resolution. Helices alpha $1(\alpha 1), \alpha 2$ and $\alpha 5$ are green (left) or slate (right). In both panels, $\alpha 3, \alpha 4$ and $\alpha 6-\alpha 8$ are yellow, cyan and pink, respectively. (B) Crystal structure of the 2:2 $\mathrm{PUMA}^{\mathrm{BH}}$ induced BCL-xL $\Delta \mathrm{C}$ dimer solved to $2.9 \AA$ resolution. The helices in the two subunits of the BCL-xL $\Delta$ C dimer are labeled $\alpha 1-\alpha 8$ and $\alpha 1^{\prime}-\alpha 8$ ', respectively. $\alpha 1, \alpha 2$ and $\alpha 5$ are green (left side of molecule) or slate (right side of molecule); $\alpha 3, \alpha 4$ and $\alpha 6-\alpha 8$ are yellow, cyan and pink, respectively; $\mathrm{PUMA}^{\mathrm{BH} 3}$ is magenta. 
Within the crystal, molecules A and B each created crystallographic dimers around 2-fold axes, and the A dimer was well resolved due to its superior electron density. Interestingly, additional unexpected electron density was observed in the initial composite omit map extending out from helix $\alpha 5$ rather than forming the reverse turn (residues 157-160) that links $\alpha 5$ to $\alpha 6$ and results in a chain reversal in the monomeric BCL-xL $\Delta \mathrm{C}$ core structure. After model building and refinement, it was clear that this region had been rearranged such that $\alpha 5$ and $\alpha 6$ formed a continuous $\alpha$-helix to generate a domain-swapped dimer in which $\alpha 6-\alpha 8$ of one BCL-xL $\Delta C$ molecule replaces $\alpha 6^{\prime}-\alpha 8^{\prime}$ of the symmetry mate (Figure 3.7B). This rearrangement provided an obvious and attractive structural explanation for our observations that the binding of the $\mathrm{PUMA}^{\mathrm{BH} 3}$ promoted dimerization of recombinant forms of BCL-xL (Figure 3.3, Figure 3.4, and Figure 3.6). Although less clear in the B molecule, the refined electron density unequivocally showed that the same rearrangement had taken place in both molecules of the crystal asymmetric unit.

We performed NMR to determine if the PUMA ${ }^{\mathrm{BH} 3}$-induced BCL-xL dimer is domain swapped in solution as seen in our crystal structure. BCL-xL $\Delta \mathrm{L} \Delta \mathrm{C}$ monomer versus heat-induced, domain-swapped BCL-xL $\Delta \mathrm{L} \Delta \mathrm{C}$ apo-dimer are distinguished by the hallmark positions of the W24 HN-indole and V135 methyl resonances (Denisov et al., 2007a), and we confirmed this (Figure 3.8). While binding of $\mathrm{PUMA}^{\mathrm{BH} 3}$ to pre-formed BCL-xL $\Delta \mathrm{L} \Delta \mathrm{C}$ apo-dimers had virtually no effect on the positions of these resonances for the dimer, addition of PUMA ${ }^{\mathrm{BH} 3}$ to BCL-xL $\Delta \mathrm{L} \Delta \mathrm{C}$ monomer induced identical shifts of both W24 and V135 resonances, characteristic of BCL-xL domain-swapped dimer formation (Denisov et al., 2007a) (Figure 3.8).

To further test the idea that the BCL-xL $\Delta \mathrm{C}$ dimer induced by $\mathrm{PUMA}^{\mathrm{BH} 3}$ in solution is indeed what we observed in the crystal structure, we introduced cysteines into BCL-xL $\Delta \mathrm{C}$ that would be in a approximate $12 \AA$ proximity only upon dimer formation (Figure 3.9A), as previously described for the alkaline $\mathrm{pH}$-induced, domain-swapped BCL-xL $\Delta C$ apo-dimer (O'Neill et al., 2006). Treatment of the mutant BCL-xL $\Delta C$ monomers with the cross-linking agent bismaleimidohexane $(\mathrm{BMH})$ produced no higher order structures; however upon addition of $\mathrm{PUMA}^{\mathrm{BH} 3}$, cross-linking revealed dimers (Figure 3.9B). Similarly, introduction of cysteines designed to prevent "opening" of $\mathrm{BCL}-\mathrm{xL} \Delta \mathrm{C}$ by formation of a disulfide bond (Figure 3.9C) prevented $\mathrm{PUMA}^{\mathrm{BH} 3}$ from inducing dimer formation in the disulfide "trapped" monomer but readily induced dimerization in the presence of reducing agent (Figure 3.9D). Together, these results strongly suggest that the BCL-xL $\Delta C$ dimer induced by $\mathrm{PUMA}^{\mathrm{BH} 3}$ in solution is the same as our crystal structure.

\section{Analyses of the BCL-xL $\Delta C \cdot P U M A^{B H 3}$ Interaction Reveal the Mechanism for PUMA-induced BCL-xL Dimerization of BCL-xL}

While the oligomeric state of the 2:2 BCL-xL $\Delta \mathrm{C} \cdot \mathrm{PUMA}^{\mathrm{BH} 3}$ complex is strikingly different from the 1:1 oligomeric state of $\mathrm{BCL}-\mathrm{xL} \Delta \mathrm{C}$ bound to the $\mathrm{BAD}^{\mathrm{BH} 3}, \mathrm{BAK}^{\mathrm{BH} 3}$ or 
Figure 3.8 The BCL-xL $\Delta C \cdot P U M A^{\text {BH3 }}$ Complex Is Domain Swapped in Solution. Regions from $1 \mathrm{D}{ }^{1} \mathrm{H}$ NMR spectra showing previously assigned (Denisov et al., 2007b) Trp indole resonances (A) and the methyl group resonance of Val 135 (B) of ${ }^{15} \mathrm{~N}-\mathrm{BCL}$ $\mathrm{xL} \Delta \mathrm{C}$ monomer (blue), ${ }^{15} \mathrm{~N}-\mathrm{BCL}-\mathrm{xL} \Delta \mathrm{C}$ apo-dimer (red), ${ }^{15} \mathrm{~N}-\mathrm{BCL}-\mathrm{xL} \Delta \mathrm{C}$ apodimer.PUMA ${ }^{\mathrm{BH} 3}$ (green), and ${ }^{15} \mathrm{~N}-\mathrm{BCL}-\mathrm{xL} \Delta \mathrm{C}$ monomer.PUMA ${ }^{\mathrm{BH} 3}$ (magenta). Twodimensional (2D) ${ }^{1} \mathrm{H}^{15}{ }^{15}$ TROSY spectra of ${ }^{15} \mathrm{~N}-\mathrm{BCL}-\mathrm{xL} \Delta \mathrm{C}$ monomer (C), ${ }^{15} \mathrm{~N}-\mathrm{BCL}-$ $\mathrm{xL} \Delta \mathrm{C}$ apo-dimer (D), ${ }^{15} \mathrm{~N}-\mathrm{BCL}-\mathrm{xL} \Delta \mathrm{C}$ apo-dimer $+\mathrm{PUMA}^{\mathrm{BH} 3}(\mathrm{E})$, and ${ }^{15} \mathrm{~N}-\mathrm{BCL}-\mathrm{xL} \Delta \mathrm{C}$ monomer $+\mathrm{PUMA}^{\mathrm{BH} 3}(\mathrm{~F})$. Tryptophan $\mathrm{HN}$-indole resonances are shown within the black dashed boxes. $(\mathrm{G})$ Overlay of 2D spectra from (E) and (F). When a red and a green peak perfectly overlay, a yellow peak is produced. The observation that all resonances appear yellow when the individual 2D spectra (E-F) are overlaid indicates that the PUMAinduced BCL-xL dimer prepared in solution was domain swapped as observed in the crystal structure. BCL-xL $\Delta \mathrm{C}$ dimer induced by $\mathrm{PUMA}^{\mathrm{BH} 3}$ in solution is the same as our crystal structure. 

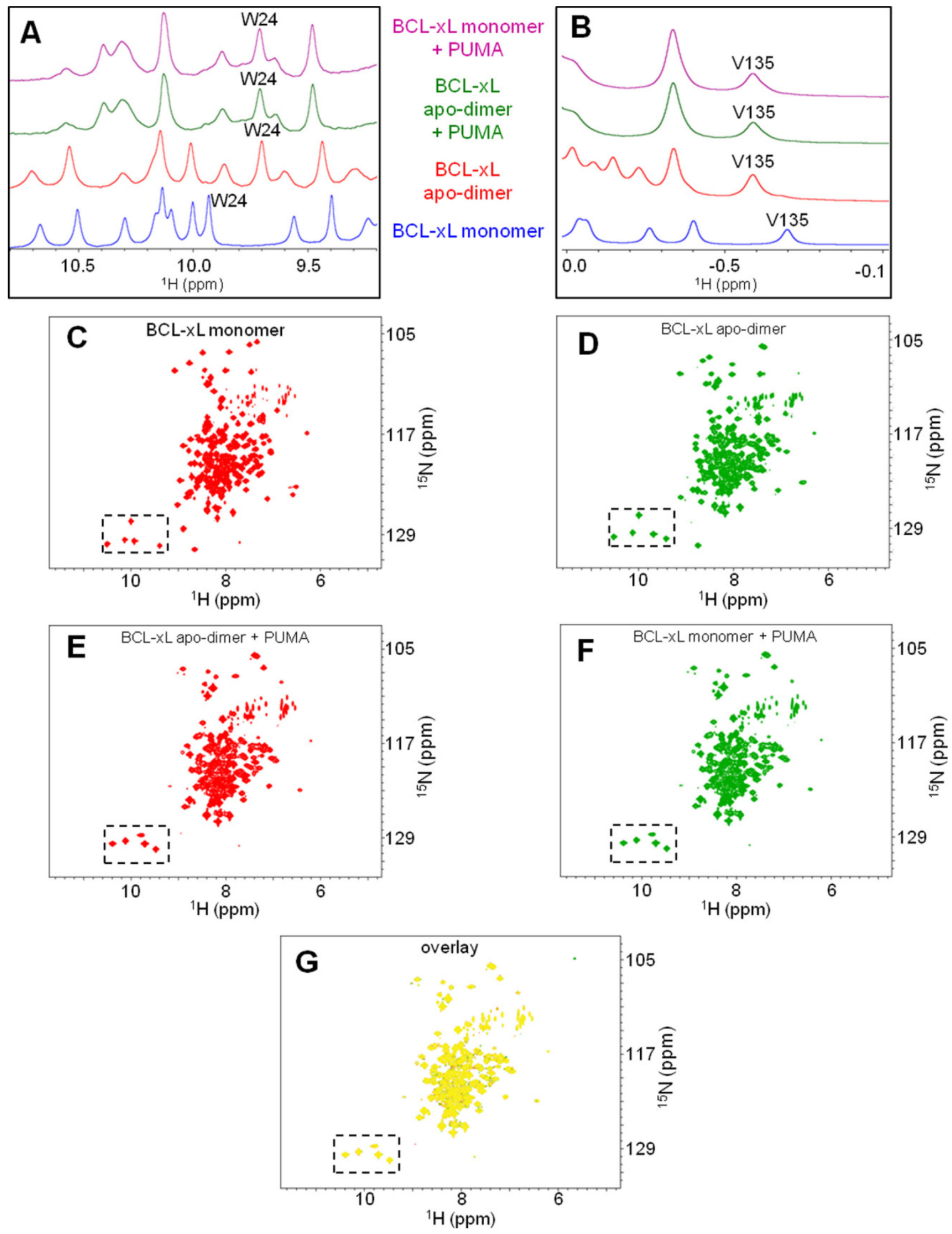
Figure 3.9 Structure-dependent Crosslinking of the PUMA-induced BCL-xL Domain Swapped Dimer Reveals that PUMA-induced BCL-xL Dimerization Occurs via Domain Swapping.

Two types of BCL-xL $\Delta \mathrm{C}$ cysteine mutants were created to probe the structure of the domain swapped dimer in solution; one type involved introduction of either an E7C or $\mathrm{N} 175 \mathrm{C}$ point mutation (A) and another type involved introduction of dual mutations, V135C and W181C (C). (A) Domain swapped dimerization of the two BCL-xL $\Delta \mathrm{C}$ point mutants mixed at equimolar ratios positions these two residues in proximity for covalent linkage by the cross linking reagent $\mathrm{BMH}$ (shown in yellow). (B) Wild type BCL-xL $\Delta \mathrm{C}$, BCL-xL $\Delta C$ E7C, BCL-xL $\Delta C$ N175C, and an equimolar ratio of BCL-xL $\Delta C$ E7C and $\mathrm{BCL}-\mathrm{xL} \triangle \mathrm{C} \mathrm{N} 175 \mathrm{C}$ were either untreated or treated with $\mathrm{BMH}$ in the presence or absence of PUMA ${ }^{\mathrm{BH} 3}$, and the proteins were analyzed by SDS-PAGE followed by western blot analysis for BCL-xL. 50\% of PUMA binding-induced dimers that form contain V135C in one polypeptide chain and $\mathrm{W} 181 \mathrm{C}$ in the other and are able to be crosslinked. PUMA does not induce dimerization of disulfide bond "trapped" BCL-xL in the absence of reducing agent. (C) A BCL-xL $\Delta \mathrm{C}$ mutant covalently locked in the monomeric state was created by introducing two cysteines, V135C and W181C, that form a solvent exposed disulfide bond (shown in yellow) linking the loop connecting $\alpha 3$ and $\alpha 4$ to $\alpha 7$. (D) The disulfide trapped monomer was treated with buffer or $\mathrm{PUMA}^{\mathrm{BH} 3}$ in the presence or absence of reducing agent, and the samples were analyzed by native PAGE and Coomassie staining to assess BCL-xL oligomeric status. BCL-xL $\Delta C$ monomer and BCL$\mathrm{xL} \Delta \mathrm{C}$ apo-dimer were used as molecular weight standards. Bismaleimidohexane (BMH), Tris[2-carboxyethyl] phosphine (TCEP). 


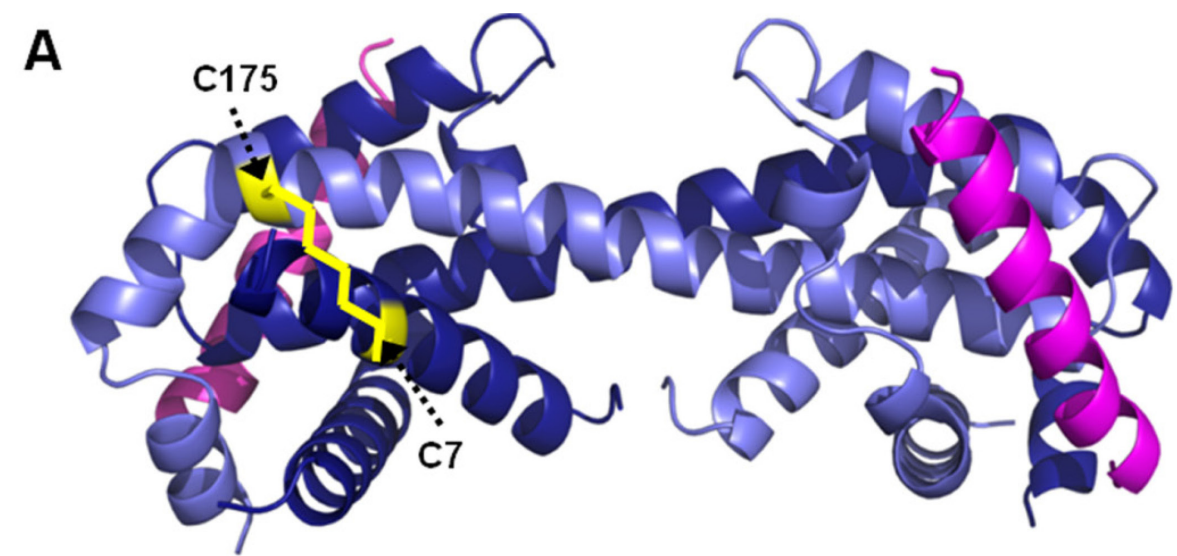

B

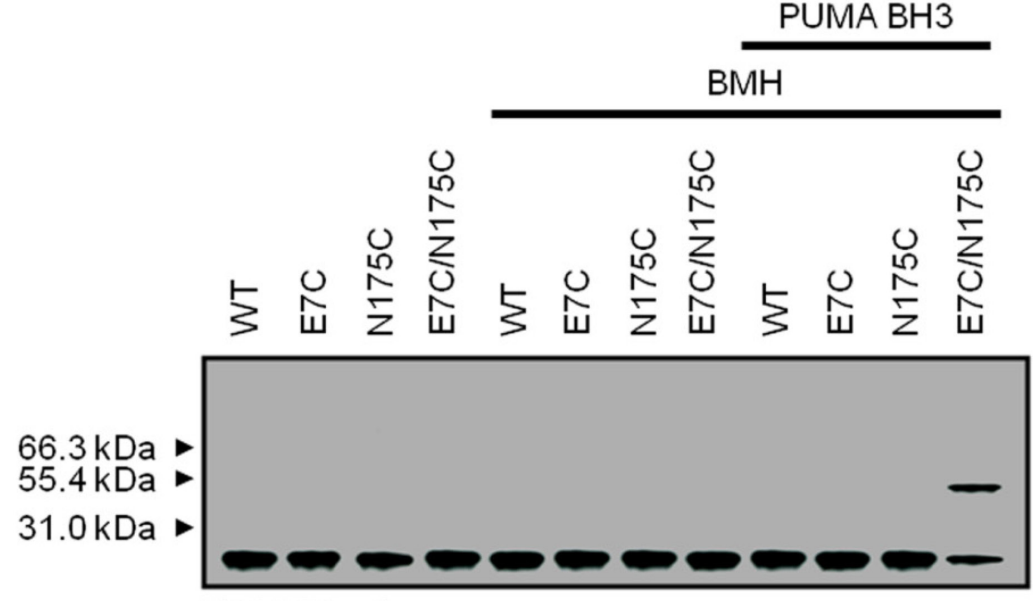

WB: BCL-xL

BCL-xL $\Delta C$ and mutants: $1 \mu \mathrm{M}$

PUMA $^{\text {BH3 }}: 2 \mu \mathrm{M}$

$\mathrm{BMH}: 5 \mu \mathrm{M}$

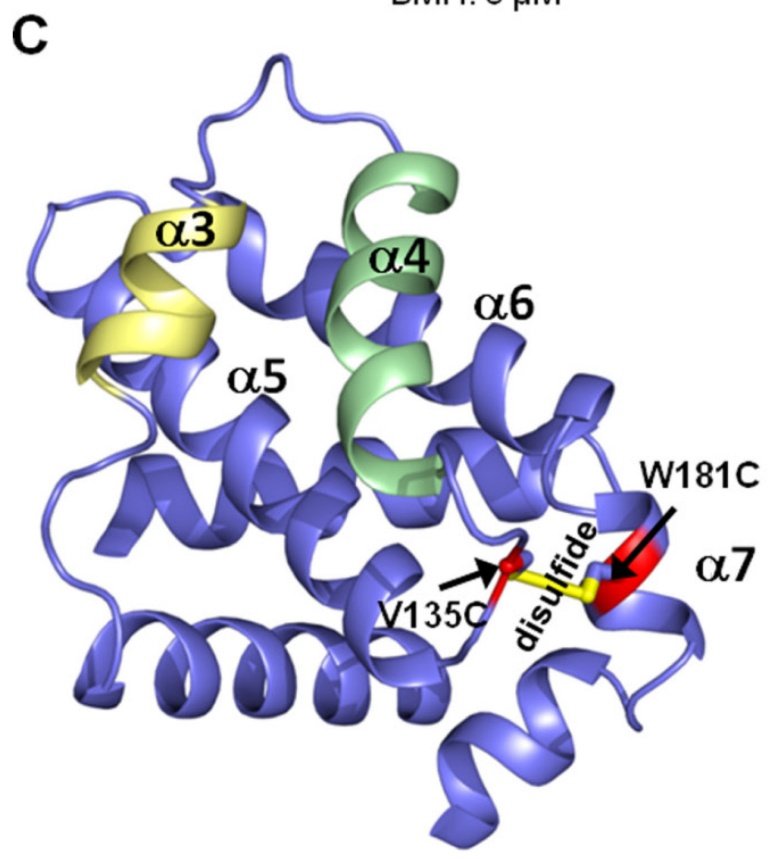

D

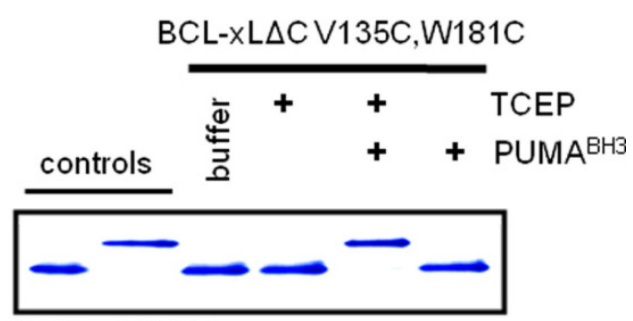

Native PAGE

BCL-xL $\triangle C$ V135C,W181C: $50 \mu \mathrm{M}$

PUMABH3: $100 \mu \mathrm{M}$

TCEP (reducing agent): $10 \mu \mathrm{M}$ 
BIM $^{\mathrm{BH} 3}$ domain peptides (Petros et al., 2000; Sattler et al., 1997),many of the important interactions between $\mathrm{BH} 3$ domain peptides and $\mathrm{BCL}-\mathrm{xL} \Delta \mathrm{C}$ were observed. $\mathrm{PUMA}^{\mathrm{BH} 3}$ bound within the surface-exposed hydrophobic groove of BCL-xL $\Delta C$, and the intermolecular interface was dominated by hydrophobic interactions in which I75, L79, R80, M2, L86 and Y90 of the PUMA peptide are particularly important (Figure 3.10). I75 was buried within a hydrophobic pocket formed by residues V126, L112, L108, F146 and F105 of BCL-xL $\Delta C$. L79 and D84 are part of the conserved L-x-x-x-x-D motif ( $\mathrm{x}=$ any amino acid) of the PUMA ${ }^{\mathrm{BH} 3}$ (Petros et al., 2004b). L79 packed into a hydrophobic groove surrounded by BCL-xL $\Delta$ C residues F146, F105, V126, F97, L130 and A142 (Figure 3.10), and D84 formed hydrogen bonds with R139 and N136, respectively (Figure 3.10). The aliphatic $\alpha, \beta$ and $\gamma$ carbons of R80 packed against L130 of BCL$\mathrm{xL} \Delta \mathrm{C}$, and the terminal guanidinium moiety formed hydrogen bonding interactions with E129 of BCL-xL $\Delta C$ (Figure 3.10). M82 packed against residues F105, A104, Y101, and $\mathrm{F} 97$ of BCL-xL $\Delta \mathrm{C}$ and was partially solvent exposed as observed in the BCL$\mathrm{xL} \Delta \mathrm{C} \cdot \mathrm{BAD}^{\mathrm{BH} 3}$ peptide structure (Petros et al., 2000). Finally, L86 and Y 90 were also partially exposed to solvent and packed against BCL-xL $\Delta \mathrm{C}$ residues $\mathrm{Y} 101, \mathrm{~F} 97, \mathrm{~A} 93$, V141, Y195 and G138; and Y195 and L194, respectively (Figure 3.10). R100 of BCL$\mathrm{xL} \Delta \mathrm{C}$ formed hydrogen bonds and/or electrostatic interactions with Q89 of the PUMA peptide, and Y101 of BCL-xL $\Delta C$ formed electrostatic interactions with D85 and the backbone carbonyl oxygen of M82 of the PUMA peptide (Figure 3.10). The residues of $\mathrm{PUMA}^{\mathrm{BH} 3}$ that mediated the hydrophobic interactions with BCL-xL $\Delta \mathrm{C}$ described above form a hydrophobic spine primarily along one face of the $\alpha$-helical structure adopted by the PUMA ${ }^{\mathrm{BH} 3}$ (Figure 3.10). Residues along the opposite face mediated an extensive hydrogen bonding and electrostatic interaction network with BCL-xL $\Delta \mathrm{C}$ (Figure 3.10).

The thermodynamic contributions of the key amino acids found to be important for the $\mathrm{BCL}-\mathrm{xL} \Delta \mathrm{C} \cdot \mathrm{PUMA}^{\mathrm{BH} 3}$ interaction in our crystal structure were determined using alanine mutagenesis and a fluorescence anisotropy binding assay (Figure 3.11). The results were fully consistent with the structural interpretations discussed above, as individual mutation of $\mathrm{PUMA}^{\mathrm{BH} 3}$ residues $\mathrm{I75}, \mathrm{L} 79, \mathrm{R} 80, \mathrm{M} 2, \mathrm{~L} 86$, and $\mathrm{Y} 90$ to alanine resulted in a decreased binding affinity for BCL-xL $\Delta \mathrm{C}$ and increased $-\Delta \Delta \mathrm{G}$ values (Figure 3.11).

Another notable feature of the BCL-xL $\Delta \mathrm{C} \cdot \mathrm{PUMA}^{\mathrm{BH} 3}$ structure is that $\mathrm{P} 116$ from BCL-xL $\Delta C$, which separates $\alpha 3$ and $\alpha 4$, was markedly displaced relative to its position in the BCL-xL $\Delta \mathrm{C}$ monomer structure (Figure 3.12). Interestingly, this proline has been reported to constitute a portion of the p53 binding epitope with BCL-xL (Petros et al., 2004a; Tomita et al., 2006). Specifically, P116 was displaced by approximately $5 \AA$ relative to its position in monomeric $\mathrm{BCL}-\mathrm{xL} \Delta \mathrm{C}$ as well as all other previously determined BH3 domain peptide-BCL-xL $\Delta \mathrm{C}$ structures (Figure 3.12).

The conformational change centered on P116 (Figure 3.12B) was associated with a $\pi$-stacking interaction between the aromatic side chain of W71 from the PUMA peptide and the imidazole ring of $\mathrm{H} 113$ from BCL-xL $\Delta \mathrm{C}$ (Figure 3.12C). This interaction is clearly visible in the $2 \mathrm{f}_{\mathrm{o}}-\mathrm{f}_{\mathrm{c}}$ electron density map (Figure 3.12D). 
A

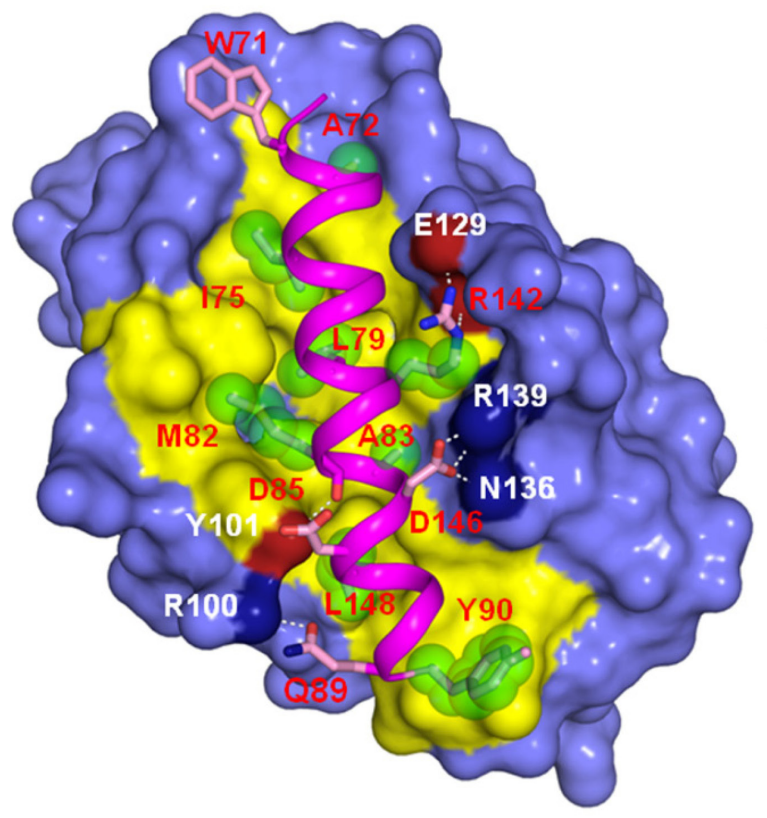

B

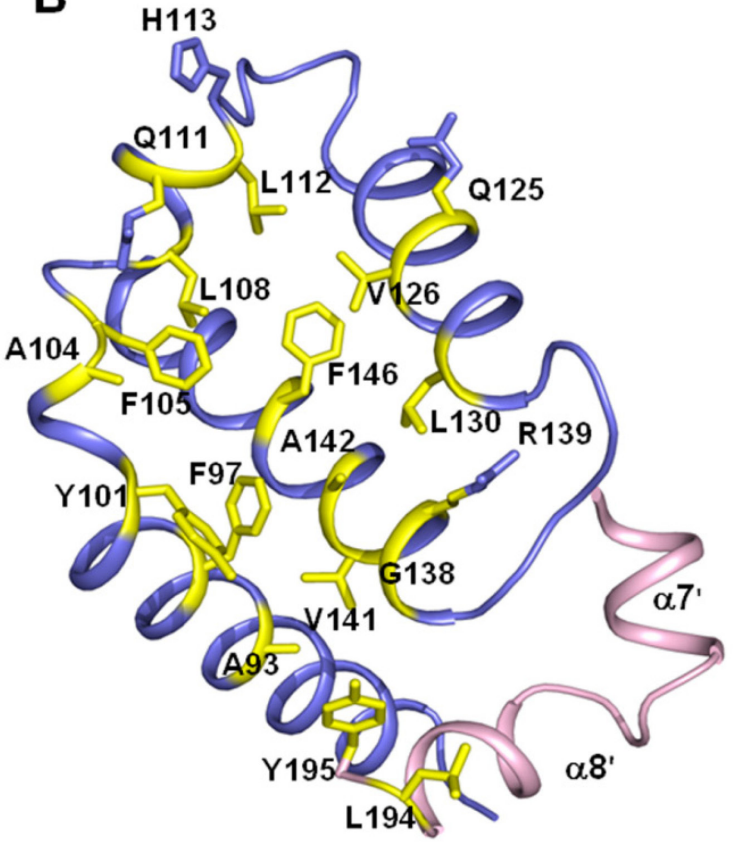

Figure 3.10 Details of the BCL-xL·PUMA ${ }^{\mathrm{BH} 3}$ Interaction.

(A) For clarity, only one side of the dimer, comprising BCL-xL $\Delta C \alpha 1-\alpha 5$ and $\alpha 6^{\prime}-\alpha 8^{\prime}$ and one $\mathrm{PUMA}^{\mathrm{BH} 3}$, is illustrated. The BCL-xL $\Delta \mathrm{C}$ hydrophobic groove is yellow, the remainder of the $\mathrm{BCL}-\mathrm{xL} \Delta \mathrm{C}$ surface is slate, and the $\mathrm{PUMA}^{\mathrm{BH} 3}$ is magenta. $\mathrm{PUMA}^{\mathrm{BH} 3}$ side chains with transparent green spheres illustrate hydrophobic residues or atoms that pack into the $\mathrm{BCL}-\mathrm{xL} \Delta \mathrm{C}$ hydrophobic groove. $\mathrm{PUMA}^{\mathrm{BH} 3}$ and $\mathrm{BCL}-\mathrm{xL} \Delta \mathrm{C}$ residues are indicated in red and white, respectively. Hydrogen bonds and electrostatic interactions are shown as white dashed lines. (B) Re-representation of $C$ showing BCL-xL $\Delta \mathrm{C}$ with the PUMA ${ }^{\mathrm{BH} 3}$ removed. The hydrophobic residues and side chain atoms of BCL-xL $\Delta \mathrm{C}$ that interact with the hydrophobic residues of PUMA ${ }^{\mathrm{BH} 3}$ are yellow. The $\alpha 7^{\prime}-\alpha 8^{\prime} \mathrm{C}-$ terminus from the juxtaposed BCL-xL $\Delta \mathrm{C}$ molecule within the dimer is rose; $\alpha 6$ ' is not illustrated for clarity. 

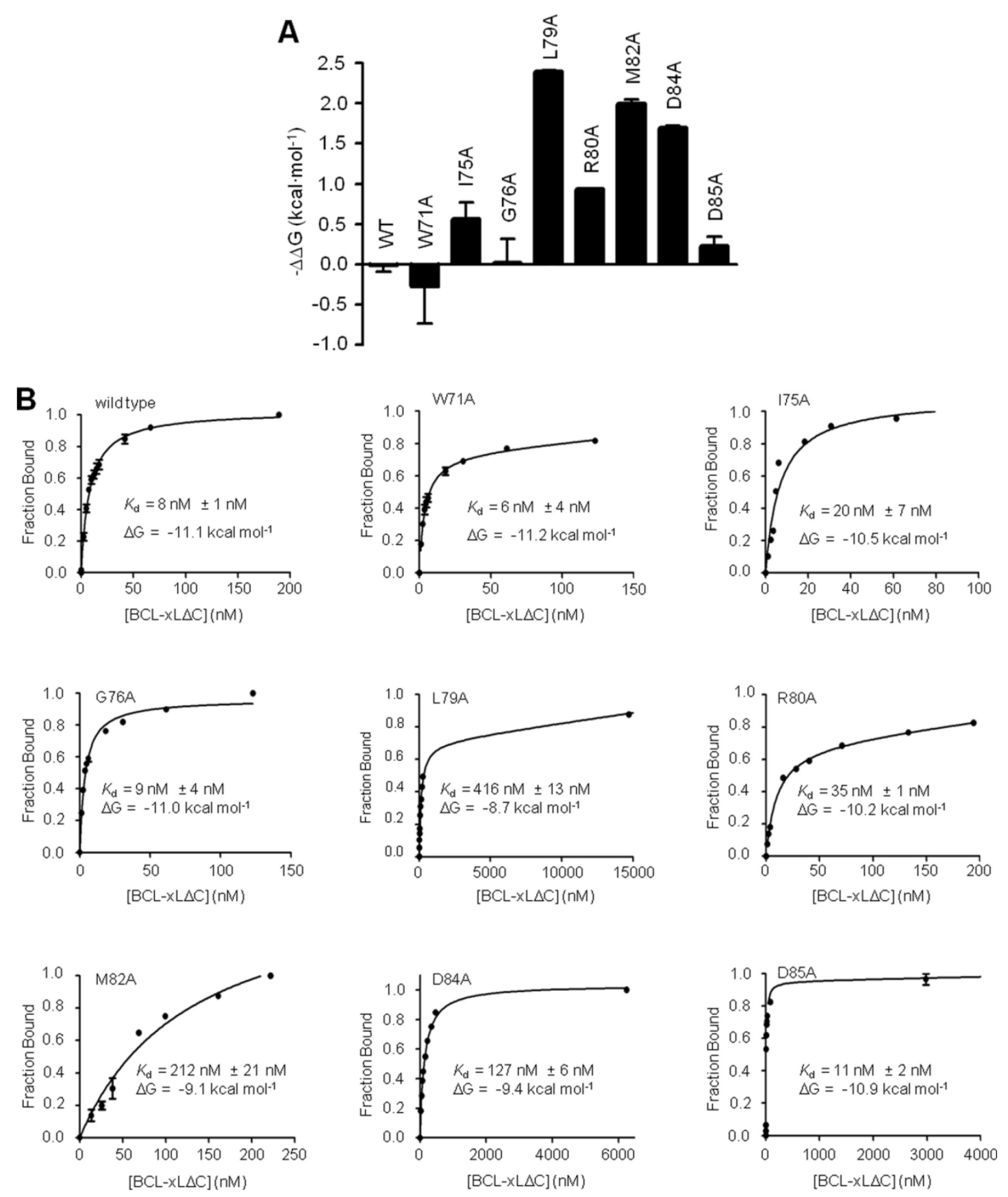

Figure 3.11 Verification of PUMA ${ }^{\mathrm{BH} 3}$ Side Chain Interactions with BCL-xL $\Delta \mathrm{C}$ by Fluorescence Anisotropy.

(A) $-\Delta \Delta \mathrm{G}$ values for the indicated PUMA ${ }^{\mathrm{BH} 3}$ alanine mutants binding to BCL-xL $\Delta \mathrm{C}$. Error bars represent the standard deviation calculated from at least two independent experiments. (B) Non-linear regression plots of the fraction of the indicated PUMA peptide bound versus BCL-xL $\Delta \mathrm{C}$ concentration. The binding affinity and value for $\Delta \mathrm{G}$ were obtained by curve fitting. Error bars and errors represent the standard deviations calculated from at least two independent experiments. 


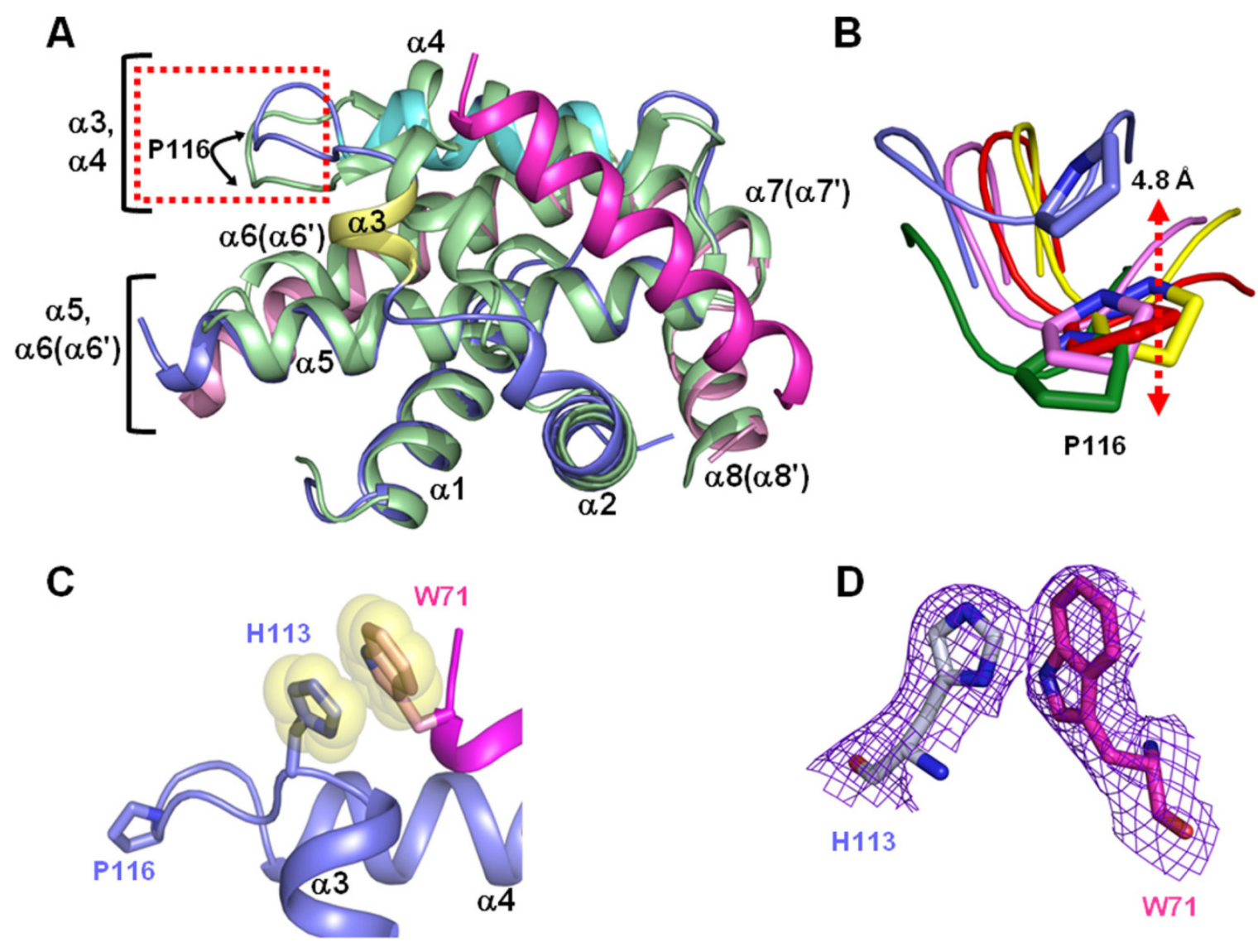

Figure 3.12 PUMA W71 Forms a $\pi$-stacking Interaction with BCL-xL H113. (A) One side of the BCL-xL $\Delta C \cdot P U M A^{\mathrm{BH} 3}$ dimer (P116 is indicated and helices $\alpha 6^{\prime}-\alpha 8^{\prime}$ are noted in parentheses) is shown superposed onto the monomeric BCL-xL $\Delta \mathrm{C}$ structure (green). The binding of $\mathrm{PUMA}^{\mathrm{BH} 3}$ perturbed BCL-xL $\Delta \mathrm{C}$ helices $\alpha 3-\alpha 4$ and influenced their interactions with $\alpha 5-\alpha 6$ ', which are opposite to the $\mathrm{PUMA}^{\mathrm{BH} 3}$ binding site. (B) Expansion of the red dashed box in $A$ after rotation of the view by approximately $90^{\circ}$ illustrating the position of P116 in the turn between BCL-xL $\Delta C \alpha 3$ and $\alpha 4$. Binding of the PUMA ${ }^{\mathrm{BH} 3}$ resulted in an approximate $5 \AA$ displacement of BCL-xL $\Delta \mathrm{C}$ P116 (slate) compared to its position in the $\mathrm{BCL}-\mathrm{xL} \Delta \mathrm{C}$ monomer (green), $\mathrm{BCL}-\mathrm{xL} \Delta \mathrm{C} \cdot \mathrm{BAD}^{\mathrm{BH} 3}$ (yellow, PDB 2BZW), BCL-xL $\triangle \mathrm{C} \cdot \mathrm{BIM}^{\mathrm{BH} 3}$ (pink, PDB 1PQ1) and BCL$\mathrm{xL} \triangle \mathrm{L} \triangle \mathrm{C} \mathrm{BAK}^{\mathrm{BH} 3}$ (red, PDB 1BXL) structures. (C) Side chains of PUMA ${ }^{\mathrm{BH} 3}$ W71 and $\mathrm{BCL}-\mathrm{xL} \Delta \mathrm{C} \mathrm{H} 113$ formed a $\pi$-stacking interaction that is associated with an approximate five Á displacement of BCL-xL $\Delta \mathrm{C}$ P116 as shown in $B$. BCL-xL $\Delta C$ is slate and $\mathrm{PUMA}^{\mathrm{BH} 3}$ is magenta. (D) $2 \mathrm{f}_{\mathrm{O}}-\mathrm{f}_{\mathrm{C}}$ electron density map surrounding PUMA ${ }^{\mathrm{BH} 3} \mathrm{~W} 71$ and BCL-xL $\Delta$ C H113 contoured at $2 \sigma$. 
That the shift in BCL-xL P116 is a consequence of the PUMA W71·BCL-xL $\mathrm{H} 113$ interaction, and thus a potential cause rather than a consequence of dimer formation, is supported by the observation that P116 in the alkaline $\mathrm{pH}$-induced BCL-xL apo-dimer is not shifted relative to that in the BCL-xL monomer (O'Neill et al., 2006). This unique structural feature of the BCL-xL $\Delta C \cdot P_{U M A}{ }^{\mathrm{BH} 3}$ complex altered the typical hydrophobic packing between the $\alpha 3-\alpha 4$ and $\alpha 5-\alpha 6$ "layers" of BCL-xL $\Delta C$ that is observed in its complexes with other $\mathrm{BH} 3$ domain peptides (Figure 3.12). We hypothesize that disruption of this $\alpha 3-\alpha 4 / \alpha 5-\alpha 6$ interface allows the $\alpha 6-\alpha 8$ structural unit to become dislodged from the $\alpha 1-\alpha 5$ core, promoting BCL-xL $\Delta C$ dimerization.

To directly test the influence of the PUMA W71·BCL-xL $\Delta$ C H113 interaction on BCL-xL dimerization, we performed a native PAGE assay using $\mathrm{BH} 3$ domain peptides from PUMA, BAD, two PUMA-BAD chimeras in which peptide residues 1-13 and 14-25 were exchanged (termed BAD-PUMA ${ }^{\mathrm{BH} 3}$ and PUMA-BAD ${ }^{\mathrm{BH} 3}$ ) and a PUMA BH3 peptide in which W71 was mutated to alanine (termed PUMA ${ }^{\mathrm{BH} 3 \mathrm{~W} 71 \mathrm{~A}}$ ) $($ Figure 3.13A). Addition of two molar equivalents of $\mathrm{PUMA}^{\mathrm{BH} 3}$ but not $\mathrm{BAD}^{\mathrm{BH} 3}$ induced BCL-xL $\Delta \mathrm{C}$ dimerization (Figure 3.13A). The BAD-PUMA ${ }^{\mathrm{BH} 3}$ peptide (which lacks the $\mathrm{W}$ residue crucial for $\pi$-stacking with BCL-xL H113) and PUMA ${ }^{\mathrm{BH} 3 \text { W71A }}$ failed to induce dimerization of BCL-xL $\Delta C$, while the PUMA-BAD ${ }^{\mathrm{BH} 3}$ peptide readily induced BCL-xL dimerization (Figure 3.13A). In this assay, wild type PUMA BH3 domain peptide or alanine point mutants were used at a concentration of $100 \mu \mathrm{M}$ except for those alanine mutants (shown in Figure 3.13B enclosed within red dashed boxes) that we previously showed by fluorescence spectroscopy had dramatically decrease affinity for BCL-xL, which were used at a concentration of $800 \mu \mathrm{M}$ to force binding through mass action. We further tested the role of BCL-xL $\triangle \mathrm{C} H 113$ in dimer formation. While $\mathrm{PUMA}^{\mathrm{BH} 3}$ induced dimer formation of BCL-xL $\Delta \mathrm{C}$, no dimerization was induced in BCL-xL $\Delta \mathrm{C}$ H113A (Figure 3.13C). Therefore, both PUMA W71 and BCL-xL H113 are necessary for PUMA-induced dimerization of BCL-xL $\Delta C$.

\section{PUMA W71 Is Required to Promote p53-dependent MOMP and Apoptosis}

We examined the consequences of the PUMA W71A mutation for binding to antiapoptotic BCL-2 proteins by ITC. While this substitution had no effect on the binding affinity of $\mathrm{PUMA}^{\mathrm{BH} 3}$ to BCL-xL $\Delta \mathrm{C}$ or BCL-xL $\Delta \mathrm{C}$ apo-dimer (Figure 3.14A and Figure 3.11), it increased binding affinity for the anti-apoptotic proteins BFL-1 $\Delta C$ and MCL$1 \Delta \mathrm{C}$ (Figure 3.14B-C). We therefore examined the role of PUMA W71 on apoptosis in cells. Wild type and puma $^{-/-}$MEFs were equally susceptible to apoptosis induced by TNF (Figure 3.15A). Introduction of PUMA or PUMA ${ }^{\mathrm{BH} 3 \mathrm{~W} 71 \mathrm{~A}}$ sensitized cells for TNFinduced apoptosis to an equivalent extent (Figure 3.15A). We next compared PUMA and

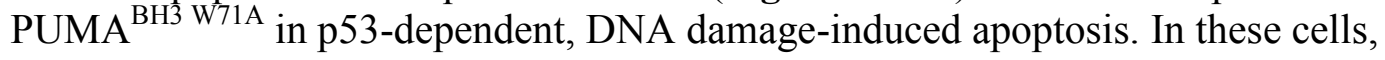


Figure 3.13 PUMA-induced Dimerization of BCL-xL is Mediated Uniquely by the $\pi$-stacking Interaction between PUMA W71 and BCL-xL H113.

PUMA $^{\mathrm{BH} 3}$ W71 was critical for BCL-xL $\Delta \mathrm{C}$ dimerization in vitro. (A) BCL-xL $\Delta \mathrm{C}$ was incubated with the indicated peptides and subjected to native PAGE followed by Coomassie staining. (B) PUMA ${ }^{\mathrm{BH} 3}$, fluoresceinated-PUMA ${ }^{\mathrm{BH} 3}$ (F-PUMA), or alanine point mutants important for interaction with BCL-xL were added to BCL-xL monomer and dimerization analyzed by native PAGE followed by Coomassie staining. F-

$\mathrm{PUMA}^{\mathrm{BH} 3}$ peptides were those used in Figure 3.11. For those peptides displaying decreased affinity for BCL-xL, $800 \mu \mathrm{M}$ of peptide was added (red boxes). The presence of fluorescein did not affect peptide-induced dimer formation. (C) BCL-xL H113 was critical for BCL-xL $\Delta \mathrm{C}$ dimerization in vitro. BCL-xL $\Delta \mathrm{C}$ and $\mathrm{BCL}-\mathrm{xL} \Delta \mathrm{C}$ H113A were incubated in the presence of $\mathrm{PUMA}^{\mathrm{BH} 3}$, and dimerization was analyzed by native PAGE followed by Coomassie staining. BCL-xL $\Delta \mathrm{C}$ monomer and BCL-xL $\Delta \mathrm{C}$ apo-dimer were used as molecular weight standards (A-C). 
A

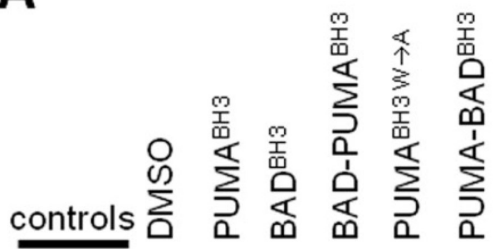

$\square-\square-\square$ dimer

Native PAGE

PUMA $^{\text {BH3 }}$ EEQWARE IGAQLRRMADDLNAQYER

$\mathrm{BAD}{ }^{\mathrm{BH} 3}$

NLWAAAQRYGRE LRRMSDEFVDSFKK

BAD-PUMA ${ }^{\text {BH3 }}$ NLWAAQRYGRELRRMADDLNAQYER

PUMA-BAD ${ }^{\text {BH } 3 ~ E E Q W A R E ~ I G A Q L R R M S D E F V D S F K K ~}$

$\mathrm{PUMA}^{\mathrm{BH} 3 \mathrm{H} \rightarrow \mathrm{A}}$ EEQAARE I GAQLRRMADDLNAQYER

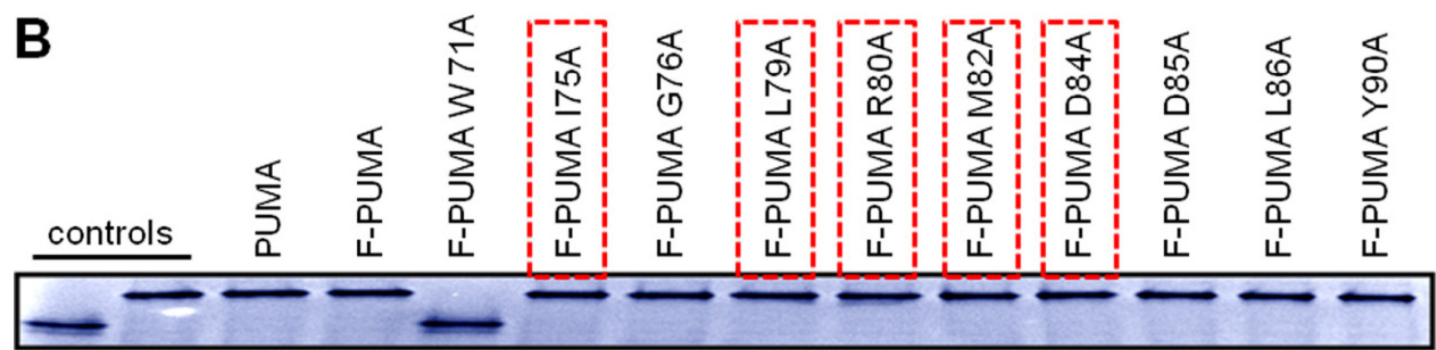

Native PAGE

BCL-xL $\Delta C: 50 \mu \mathrm{M}$

BH3 peptides: 100 or $800 \mu \mathrm{M}$

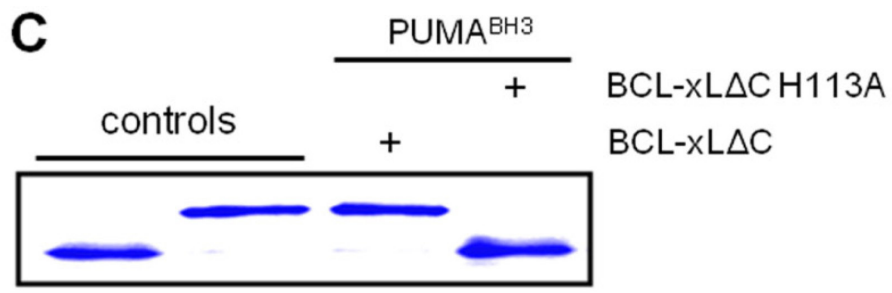

Native PAGE

$B C L-x L \Delta C \& B C L-x L \Delta C H 113 A: 50 \mu \mathrm{M}$

BH3 peptides: $100 \mu \mathrm{M}$ 


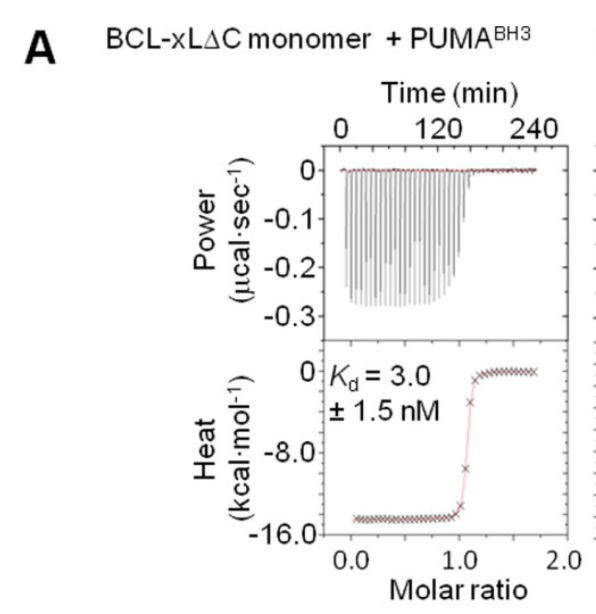

BCL-xL $\Delta C$ apo-dimer + PUMABH3
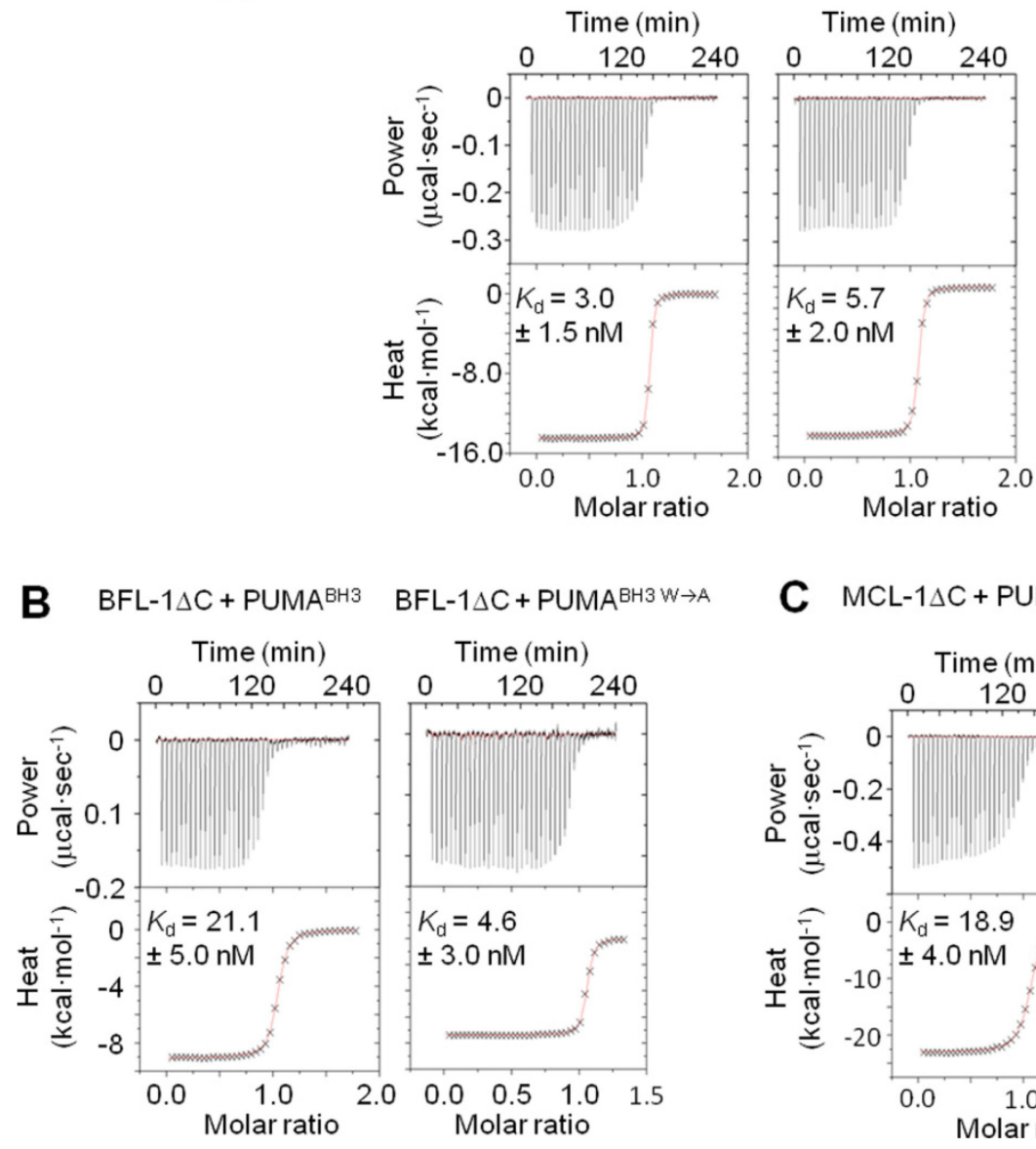

C MCL-1 $\triangle \mathrm{C}+\mathrm{PUMA}{ }^{\mathrm{BH} 3} \quad \mathrm{MCL}-1 \Delta \mathrm{C}+\mathrm{PUMA} \mathrm{PH}^{\mathrm{B}} \mathrm{W} \rightarrow \mathrm{A}$

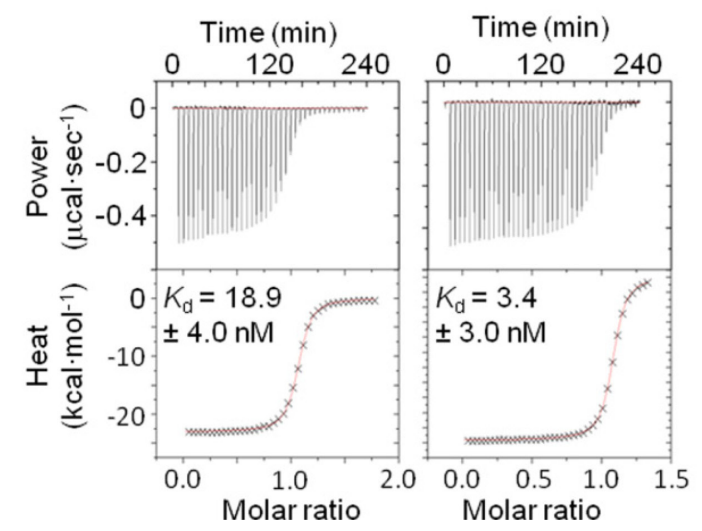

Figure 3.14 The W71A Point Mutation in PUMA ${ }^{\mathrm{BH} 3}$ Does Not Affect Binding to Other Anti-apoptotic BCL-2 Family Proteins.

(A) The PUMA ${ }^{\mathrm{BH} 3}$ binds both BCL-xL $\Delta \mathrm{C}$ monomer and BCL-xL $\Delta \mathrm{C}$ apo-dimer with equal affinity. ITC binding isotherms for PUMA ${ }^{\mathrm{BH} 3}(100 \mu \mathrm{M})$ titrated into a solution of BCL-xL $\Delta \mathrm{C}(10 \mu \mathrm{M})$ (left panel) or BCL-xL $\Delta \mathrm{C}$ apo-dimer $(10 \mu \mathrm{M})$ (right panel). ITC binding isotherms for PUMA ${ }^{\mathrm{BH} 3}$ and $\mathrm{PUMA}^{\mathrm{BH} 3 \mathrm{~W} 133 \mathrm{~A}}(100 \mu \mathrm{M})$ titrated into a solution of BFL-1 $\Delta \mathrm{C}(10 \mu \mathrm{M})(\mathrm{B})$ or MCL-1 $\Delta \mathrm{C}(10 \mu \mathrm{M})(\mathrm{C})$. 
A
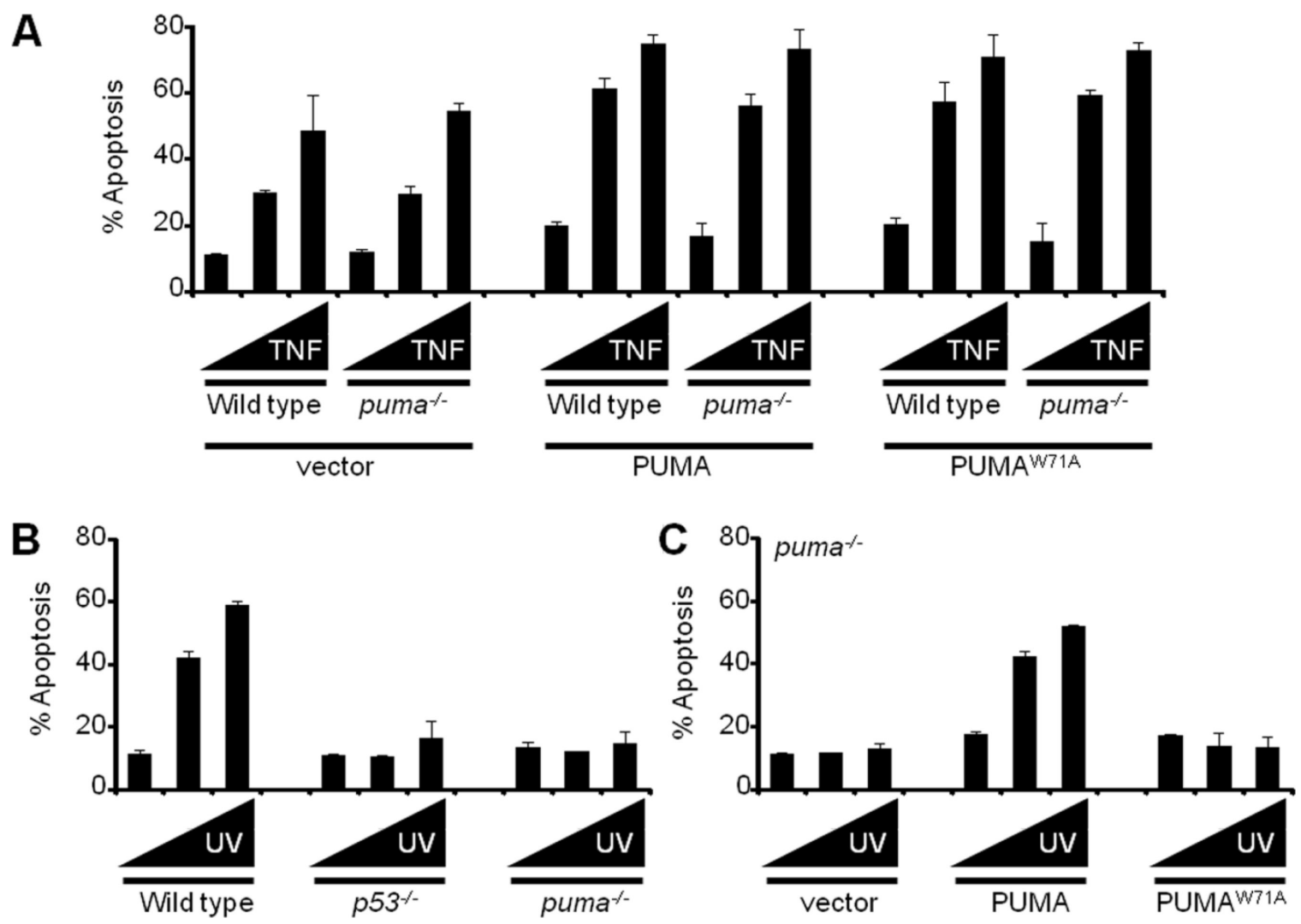

Figure 3.15 PUMA W71 Specifically Promotes DNA Damage-induced Apoptosis. (A) Wild type and puma $^{-/-}$MEFs were transiently transfected with pCMVneoBam, pCMV5neoBam-FLAG-PUMA or pCMV5neoBam-FLAG-PUMA W71A and allowed to recover for $24 \mathrm{~h}$, treated with $\operatorname{TNF}(0,5 \& 10 \mathrm{ng} / \mathrm{ml})$ and cycloheximide $(10 \mu \mathrm{g} / \mathrm{ml})$ for 6 hours and analyzed by AnnexinV-PE staining and flow cytometry for apoptosis. US9GFP was co-transfected and only GFP positive cells were analyzed. (B) Wild type, $p 53^{-/-}$ and puma $^{-/-}$MEFs were treated with UV irradiation $\left(0,2.5 \& 5 \mathrm{~mJ} / \mathrm{cm}^{2}\right)$ and analyzed 24 hours later by AnnexinV-PE staining and flow cytometry for apoptosis. (C) puma ${ }^{-/-} \mathrm{MEFs}$ were transiently transfected with pCMVneoBam, pCMVneoBam-FLAG-PUMA or pCMVneoBam-FLAG-PUMA W71A (PUMA ${ }^{\text {W71A }}$ ), recovered for $24 \mathrm{~h}$, treated with UV irradiation $\left(0,2.5 \& 5 \mathrm{~mJ} / \mathrm{cm}^{2}\right)$ and analyzed 24 hours later by AnnexinV-PE staining and flow cytometry for apoptosis. US9-GFP was co-transfected, and only GFP positive cells were analyzed. Error bars represent the standard deviation calculated from at least three independent experiments (A-C). 
apoptosis required both p53 and PUMA (Figure 3.15B), as described (Jeffers et al., 2003; Villunger et al., 2003). Strikingly, while introduction of PUMA ino puma $^{-/-}$cells effectively restored sensitivity to UV-induced apoptosis, introduction of PUMA ${ }^{\mathrm{BH} 3}$ W71A did not (Figure 3.15C). Consistent with our finding that PUMA $^{\mathrm{BH} 3}$ and PUMA ${ }^{\mathrm{BH} 3}$ W71A bind to BCL-xL equivalently (Figure 3.11), co-immunoprecipitation of ectopically expressed PUMA or PUMA ${ }^{\mathrm{BH} 3 \mathrm{~W} 71 \mathrm{~A}}$ co-precipitated endogenous BCL-xL in both cases (Figure 3.16). Intriguingly, only PUMA W71A but not PUMA co-precipitated cytosolic p53 in cells exposed to UV (Figure 3.16). We will return to this observation below.

We then examined the effects of PUMA on the oligomeric state of BCL-xL in cells. Wild type or puma $^{-/-}$MEFs were subjected to UV irradiation, which induces PUMA expression, and endogenous BCL-xL was analyzed by native PAGE and western analysis (Figure 3.17). As controls, we compared recombinant BCL-xL $\Delta \mathrm{C}$ monomer, BCL-xL $\Delta \mathrm{C}$ apo-dimer, and BCL-xL $\Delta C \cdot P U M A$ dimer. Endogenous BCL-xL in wild type MEFs exposed to UV appeared as a higher order, PUMA-bound dimer, while in puma ${ }^{-/}$cells, BCL-xL remained monomeric (compare left and right panels, Figure 3.17). Ectopic expression of PUMA in puma $^{-/-}$cells similarly resulted in the generation of the higher order, PUMA-bound BCL-xL dimer, while PUMA W71A bound to BCL-xL with a 1:1 ratio (Figure 3.17).

Since PUMA occupies the same binding groove in BCL-xL that is bound by all other BH3-only proteins, it was not obvious what effect the specific induction of BCL-xL dimerization might have on apoptotic signaling and cell fate. We therefore interrogated protein protein interactions that might be affected by such dimerization. It has been previously shown that $\mathrm{p} 53^{\mathrm{UVIP}}$ is bound by BCL-xL, and that disruption of the BCL$\mathrm{xL} \cdot \mathrm{p} 53^{\mathrm{UVIP}}$ complex by PUMA releases $\mathrm{p} 53^{\mathrm{UVIP}}$ to activate BAX and induce MOMP in isolated mitochondria, as detected by the release of cytochrome c (Chipuk et al., 2005a). Mitochondria from poly-dIdC-treated MxCre $b a k^{-/-} b a x^{f /-}$ mice (referred hereafter as $b a k^{-/-}$ $b a x^{-/}$) did not undergo MOMP in response to C8-BID, p53 ${ }^{\text {UVIP }}$, PUMA or BAX ; however, $b a x^{--} b_{a x}$ mitochondria released cytochrome $\mathrm{c}$ when exposed to BAX plus C8BID or p53 ${ }^{\mathrm{UVIP}}$ (but not PUMA or PUMA ${ }^{\mathrm{BH} 3}$ ) (Figure 3.18), as previously described (Chipuk et al., 2008). We then compared the ability of PUMA or PUMA ${ }^{\mathrm{BH} 3}$ and other derepressor BH3 domain peptides to disrupt a BCL-XLAC $\cdot \mathrm{p} 53^{\mathrm{UVIP}}$ complex and induce BAX-dependent MOMP (Figure 3.19). $B a k^{-/-} b a x^{-/-}$mitochondria were incubated with BAX plus BCL-xL $\Delta C \cdot p 53^{\text {UVIP }}$. In the presence of PUMA or PUMA ${ }^{\mathrm{BH} 3}$, cytochrome $\mathrm{c}$ release occurred, and p53 did not co-precipitate with BCL-xL $\Delta \mathrm{C}$ indicating it had been released from BCL-xL (Figure 3.19A). In contrast, none of the other de-repressor BH3 domain peptides disrupted the BCL-xL $\Delta \mathrm{C} \cdot \mathrm{p} 53^{\mathrm{UVIP}}$ complex or promoted BAX-dependent MOMP (Figure 3.19A). Similarly, failed to disrupt the BCL-xL $\Delta C \cdot p 53^{\mathrm{UVIP}}$ (Figure 3.19B). The direct activator BH3-only peptides were not tested as in Figure 3.19A, as these BH3 domain peptides directly promote BAX-dependent MOMP (Kuwana et al., 2005). As mentioned above, all BH3 domain peptides were capable of binding to BCL$\mathrm{xL}$, as determined by ITC (Figure 3.5). Thus, the unique ability of PUMA to disrupt the BCL-xL-p53 ${ }^{\text {UVIP }}$ complex could not be ascribed to differences in affinity between PUMA and all other $\mathrm{BH} 3$-only proteins, as some of these $\mathrm{BH} 3$ domain peptides exhibited binding affinity similar to or greater than that of $\mathrm{PUMA}^{\mathrm{BH} 3}$ (e.g., $\mathrm{BIM}^{\mathrm{BH} 3}$ and $\mathrm{BAD}^{\mathrm{BH} 3}$ ). 


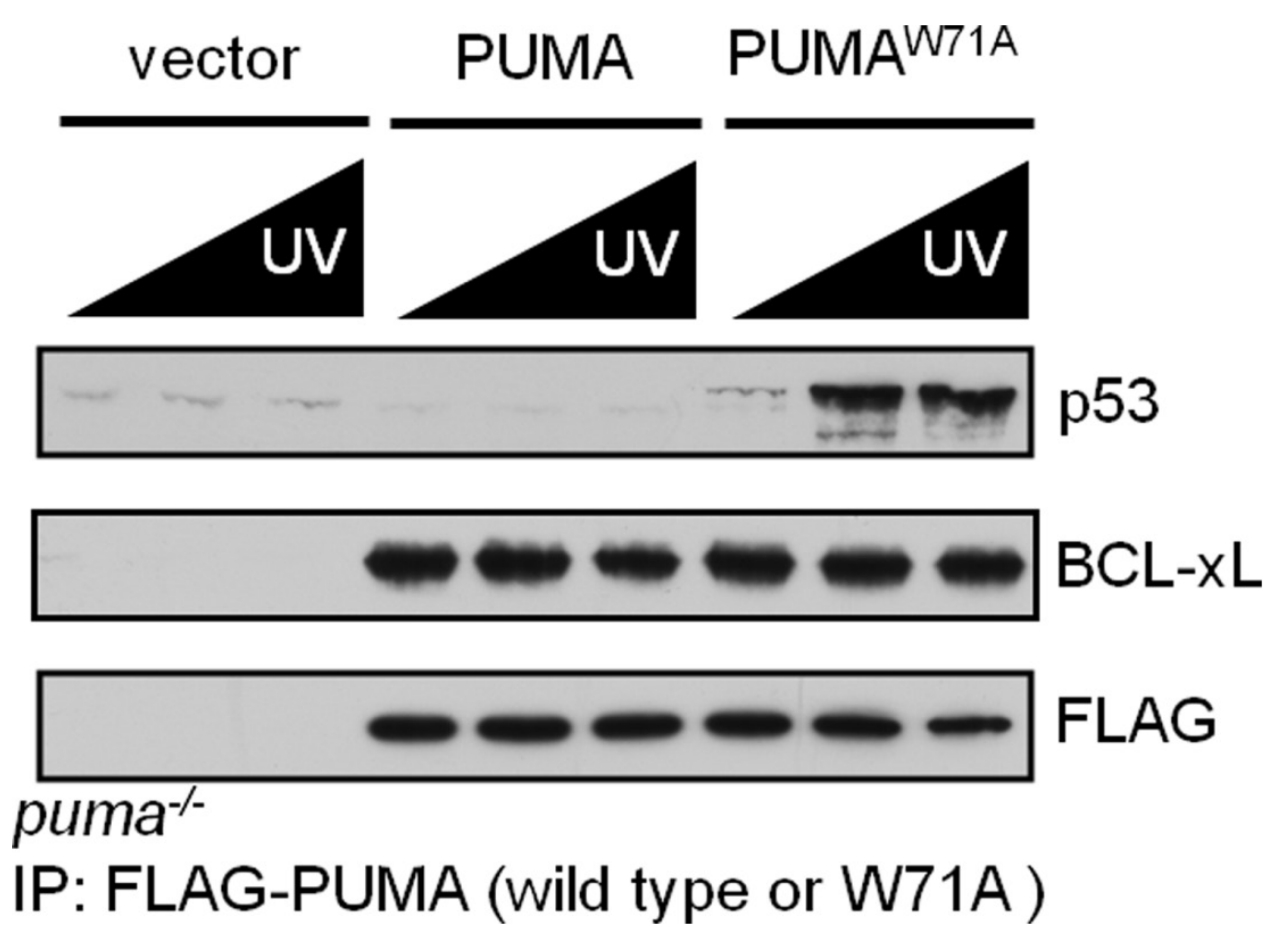

Figure 3.16 P53 and PUMA Bind Different Solvent-exposed Surfaces on BCL-xL. Lysates from Figure 3.15C above were subjected to co-immunoprecipitation with antiFLAG and analyzed by SDS-PAGE and western blot for FLAG-PUMA (wild type or W71A), BCL-xL and p53. 


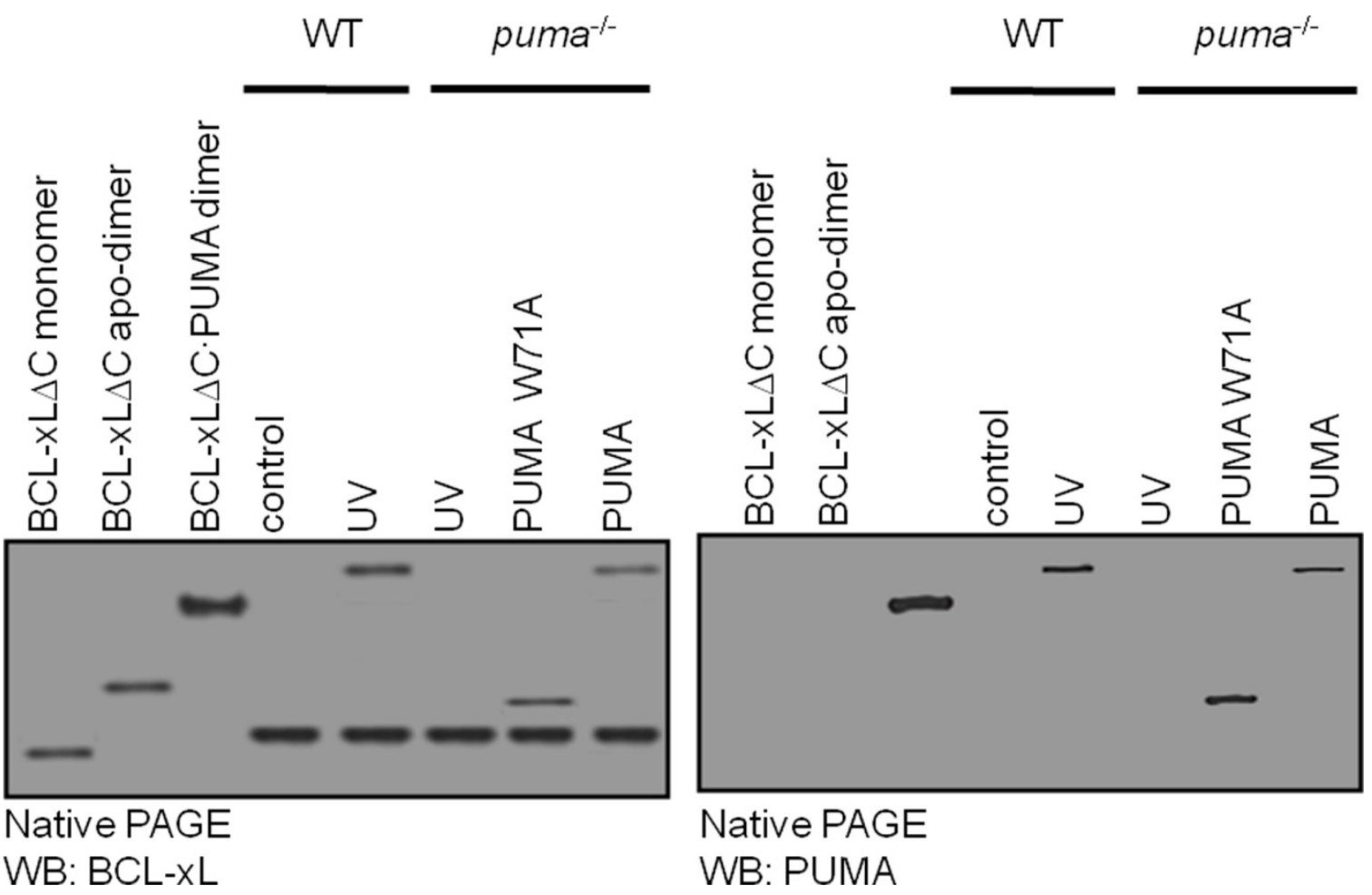

Figure 3.17 PUMA Induces BCL-xL Dimerization in Cells.

Wild type and puma $^{-/-}$MEFs (mouse embryonic fibroblasts) were treated with UV irradiation $\left(2.5 \mathrm{~mJ} / \mathrm{cm}^{2}\right)$; and puma $^{-/-}$MEFs were transiently transfected with pCMVneoBam-FLAG-PUMA or pCMVneoBam-FLAG-PUMA ${ }^{\text {W133A }}$. Lysates were subjected to native-PAGE and western blot analysis for BCL-xL dimerizationand PUMA association. BCL-xL $\Delta \mathrm{C}$ monomer, BCL-xL $\Delta \mathrm{C}$ apo-dimer and BCL-xL $\Delta \mathrm{C}$ PUMA-dimer were used as native PAGE controls. 

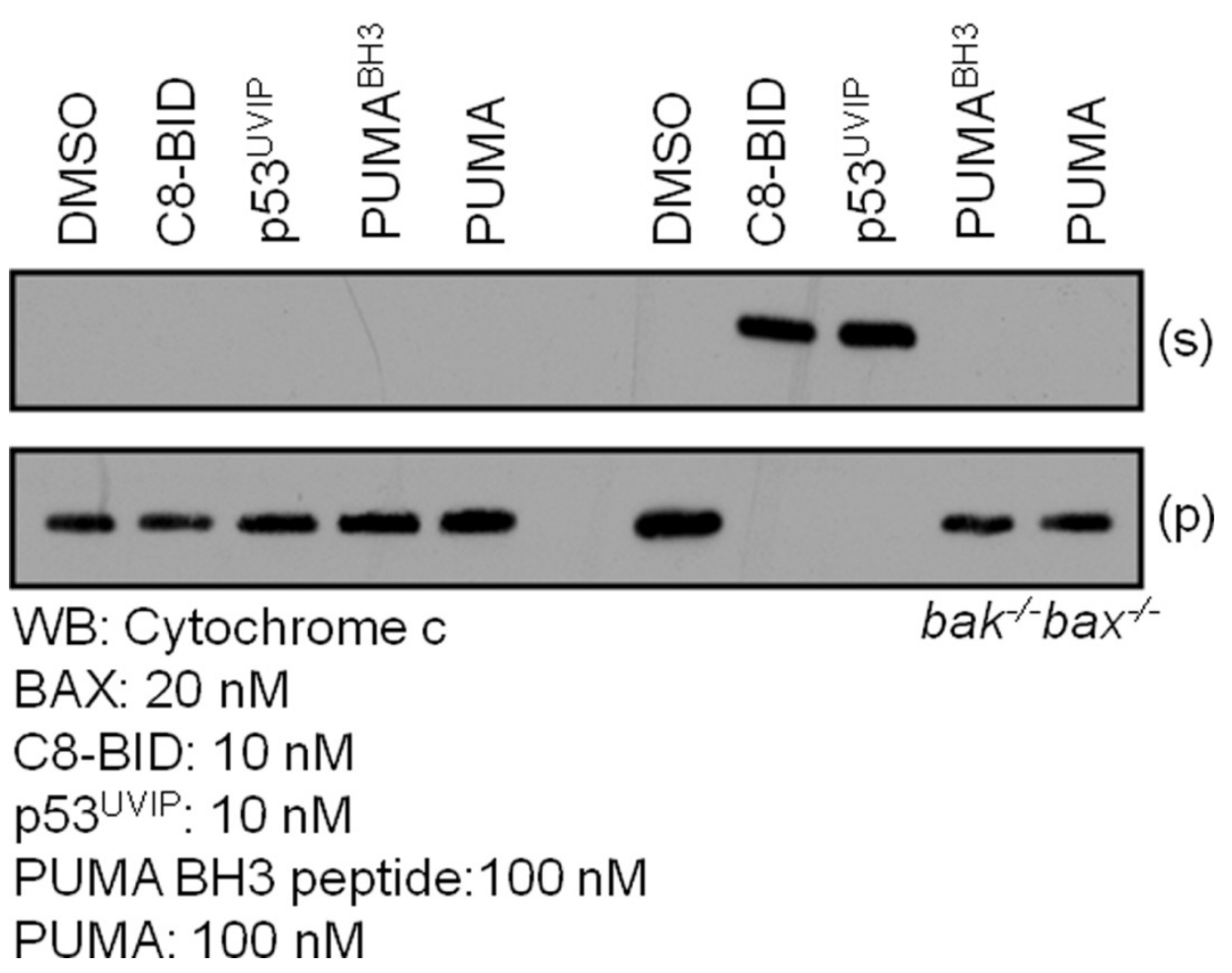

Figure 3.18 C8-BID and p53 Exhibit Direct Activation of BAX and Induction of MOMP, whereas PUMA Does Not.

Purified mitochondria from $b a k^{-/-} b a x^{-/-}$liver were treated with $\mathrm{p} 53^{\mathrm{UVIP}}(10 \mathrm{nM})$ or C8BID $(10 \mathrm{nM})$ in the presence or absence of BAX $(20 \mathrm{nM})$ for one $\mathrm{hr}$ at $37^{\circ} \mathrm{C}$ before fractionation, SDS-PAGE and western blot analyses for cytochrome c. C8-BID was a positive control for direct activator function. 

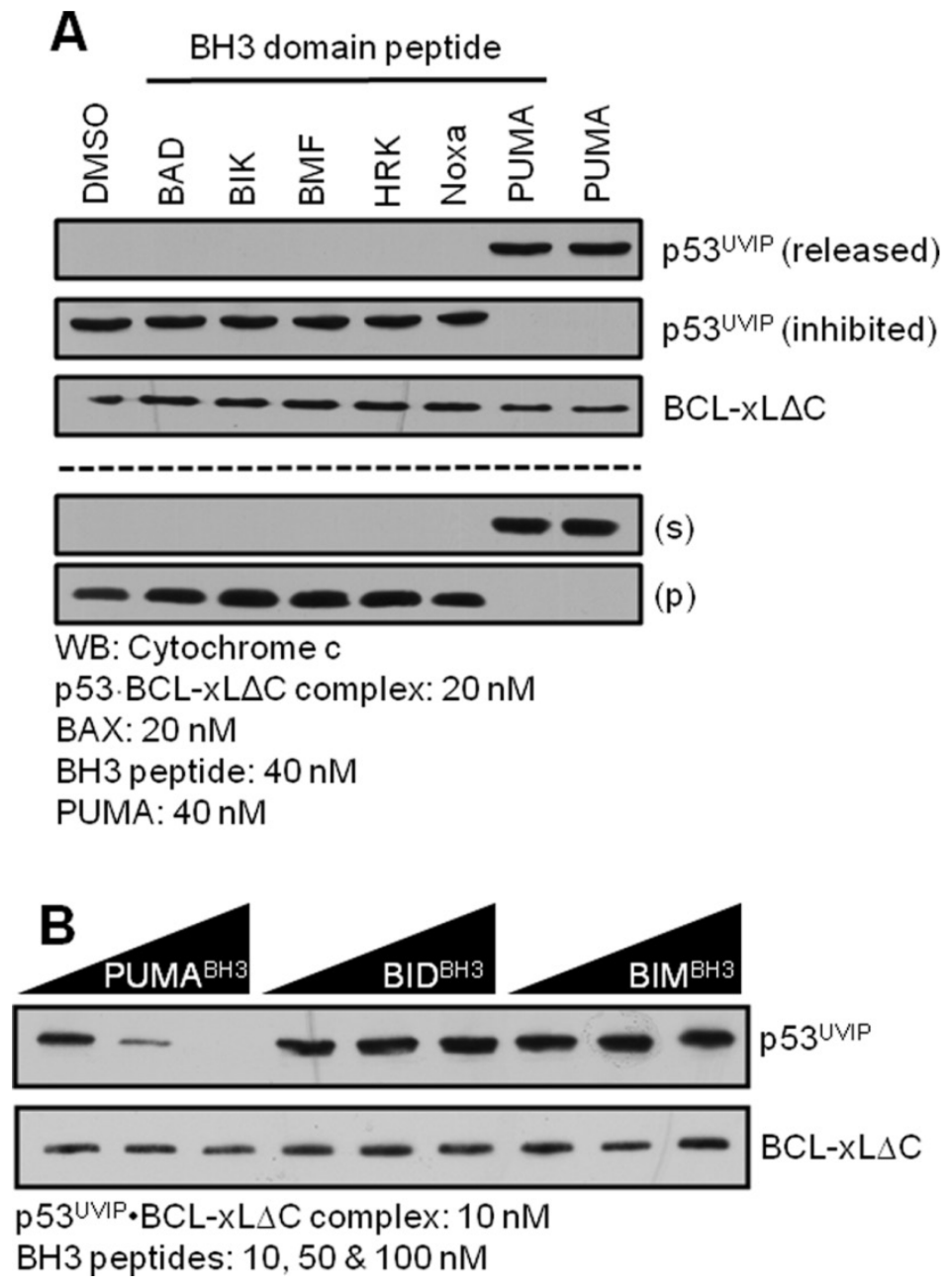

Figure 3.19 PUMA and PUMA ${ }^{\mathrm{BH} 3}$ Uniquely Release $\mathrm{p53}^{\mathrm{UVIP}}$ from BCL-xL $\Delta \mathrm{C}$. (A) $10 \mathrm{nM}$ complexes of BCL-xL $\Delta \mathrm{C} \cdot \mathrm{p} 53^{\mathrm{UVIP}}$ were combined with $b a k^{-1}$ bax $^{-/-}$liver mitochondria in the presence of BAX $(20 \mathrm{nM})$ and indicated de-repressor BH3 domain peptides or PUMA $(100 \mathrm{nM})$ for one hour at $37^{\circ} \mathrm{C}$ before fractionation, SDS-PAGE and western blot analyses for $\mathrm{p} 53^{\mathrm{UVIP}}, \mathrm{BCL}-\mathrm{xL} \Delta \mathrm{C}$ and cytochrome c. (B) $10 \mathrm{nM}$ complexes of BCL-xL $\Delta C \cdot p 53^{\mathrm{UVIP}}$ were combined with PUMA ${ }^{\mathrm{BH} 3}(10,50$, and $100 \mathrm{nM})$ or the indicated direct activator $\mathrm{BH} 3$ domain peptides $(10,50$, and $100 \mathrm{nM})$ and $\mathrm{BCL}-\mathrm{xL}$ was then isolated by affinity chromatography. Associated p53 and BCL-xL were detected by western blot. 
We next investigated the role of PUMA W71 in de-repression of C8-BID or $\mathrm{p} 53^{\mathrm{UVIP}}$ from BCL-xL $\Delta \mathrm{C}$ to promote BAX activation and MOMP. Bak $k^{-/} b a x^{-/-}$ mitochondria underwent complete cytochrome c release when treated with BAX and either C8-BID or $\mathrm{p} 53^{\mathrm{UVIP}}$, and BCL-xL $\Delta \mathrm{C}$ inhibited the release induced by both direct activator proteins (Figure 3.20). PUMA protein, $\mathrm{PUMA}^{\mathrm{BH} 3}, \mathrm{PUMA}^{\mathrm{BH} 3 \mathrm{~W} 1 \mathrm{~A}}$, PUMA$\mathrm{BAD}^{\mathrm{BH} 3}$ and $\mathrm{BAD}^{\mathrm{BH} 3}$ all failed to exhibit direct activator activity when added with $\mathrm{BAX}$ (Figure 3.20A); however, all of these completely de-repressed C8-BID from BCL-xL $\Delta C$ to induce cytochrome $\mathrm{c}$ release (Figure 3.20A). The ability of the chimeric peptides to displace C8-BID from BCL-xL $\triangle \mathrm{C}$ confirmed that these peptides bound within the hydrophobic groove of BCL-xL $\Delta \mathrm{C}$ similarly to other $\mathrm{BH} 3$ domain peptides. In contrast, PUMA protein, $\mathrm{PUMA}^{\mathrm{BH} 3}$ and PUMA-BAD ${ }^{\mathrm{BH} 3}$ promoted $\mathrm{p}^{2} 3^{\mathrm{UVIP}}$-dependent cytochrome c release, but $\mathrm{PUMA}^{\mathrm{BH} 3 \mathrm{~W} 71 \mathrm{~A}}$ and $\mathrm{BAD}^{\mathrm{BH} 3}$ did not (Figure 3.20B); therefore, PUMA $\mathrm{W} 71$ was essential for dissociation of $\mathrm{p} 53^{\mathrm{UVIP}}$ from BCL-xL $\Delta \mathrm{C}$.

Both PUMA-mediated BCL-xL dimerization and $\mathrm{p} 53^{\mathrm{UVIP}}$ de-repression required the presence of W71 in PUMA, and therefore it was likely that PUMA-induced BCL-xL dimerization promoted p53-dependent apoptosis by disrupting the sequestration of cytosolic p53 by BCL-xL. We tested this idea by examining the effects of PUMA on dimerization of BCL-xL $\Delta \mathrm{C}$ under these conditions. As in our previous experiments, p53 ${ }^{\mathrm{UVIP}}$ and BAX cooperated to promote cytochrome $\mathrm{c}$ release that was inhibited by $\mathrm{BCL}-\mathrm{xL} \triangle \mathrm{C}$ monomer (Figure 3.21A). Addition of PUMA or PUMA ${ }^{\mathrm{BH} 3}$ disrupted the p53 ${ }^{\mathrm{UVIP}} \cdot \mathrm{BCL}-\mathrm{xL} \Delta \mathrm{C}$ complex to induce cytochrome c release (Figure 3.20A). BAD ${ }^{\mathrm{BH} 3}$ failed to de-repress this complex to promote cytochrome $\mathrm{c}$ release and BCL-xL $\triangle \mathrm{C}$ apodimer did not inhibit p53 ${ }^{\mathrm{UVIP}}$-induced, BAX-mediated cytochrome c release (Figure 3.21A). Native PAGE and western blot analysis following the above treatments revealed that PUMA protein and PUMA ${ }^{\mathrm{BH} 3}$ induced dimerization of BCL-xL $\Delta \mathrm{C}$ monomers within the MOMP reactions, and this effect correlated with cytochrome c release (Figure 3.21A -B).

\section{Dimeric BCL-xL $\Delta C$ Does Not Inhibit p53-dependent MOMP}

Based on our structural findings, we hypothesized that PUMA-induced dimerization of BCL-xL provides a mechanism for modulating the association between cytoplasmic $\mathrm{p} 53$ and BCL-xL, and therefore the direct activator function of cytoplasmic p53. To test this hypothesis, co-immunoprecipitation experiments were performed. BCL$\mathrm{xL} \Delta \mathrm{C}$ monomer or BCL-xL $\Delta \mathrm{C}$ apo-dimer were incubated with baculovirus-expressed, full-length human p53 (p53 ${ }^{\mathrm{Bac}}$ ), and this complex was co-immunoprecipitated with antibodies against either 553 or BCL-xL $\Delta \mathrm{C}$, followed by SDS-PAGE and western blot analysis (Figure 3.22A-B). In all cases, BCL-xL $\Delta C$ monomer bound to $\mathrm{p} 53^{\mathrm{Bac}}$, consistent with earlier results using endogenous BCL-xL and stress-induced p53 (Chipuk et al., 2005b; Chipuk et al., 2004). In contrast, BCL-xL $\Delta$ C apo-dimer did not associate withp53 ${ }^{\mathrm{Bac}}$ (Figure 3.22A-B). Further, the interaction of BCL-xL $\Delta \mathrm{C}$ monomer or BCL$\mathrm{xL} \Delta \mathrm{C}$ apo-dimer with $\mathrm{p} 53^{\mathrm{Bac}}$ was quantitatively analyzed in solution using ITC. BCL$\mathrm{xL} \Delta \mathrm{C}$ monomer bound to p53 with a $K_{\mathrm{d}}$ value of $254 \mathrm{nM}$ (Figure 3.22C). In contrast, 

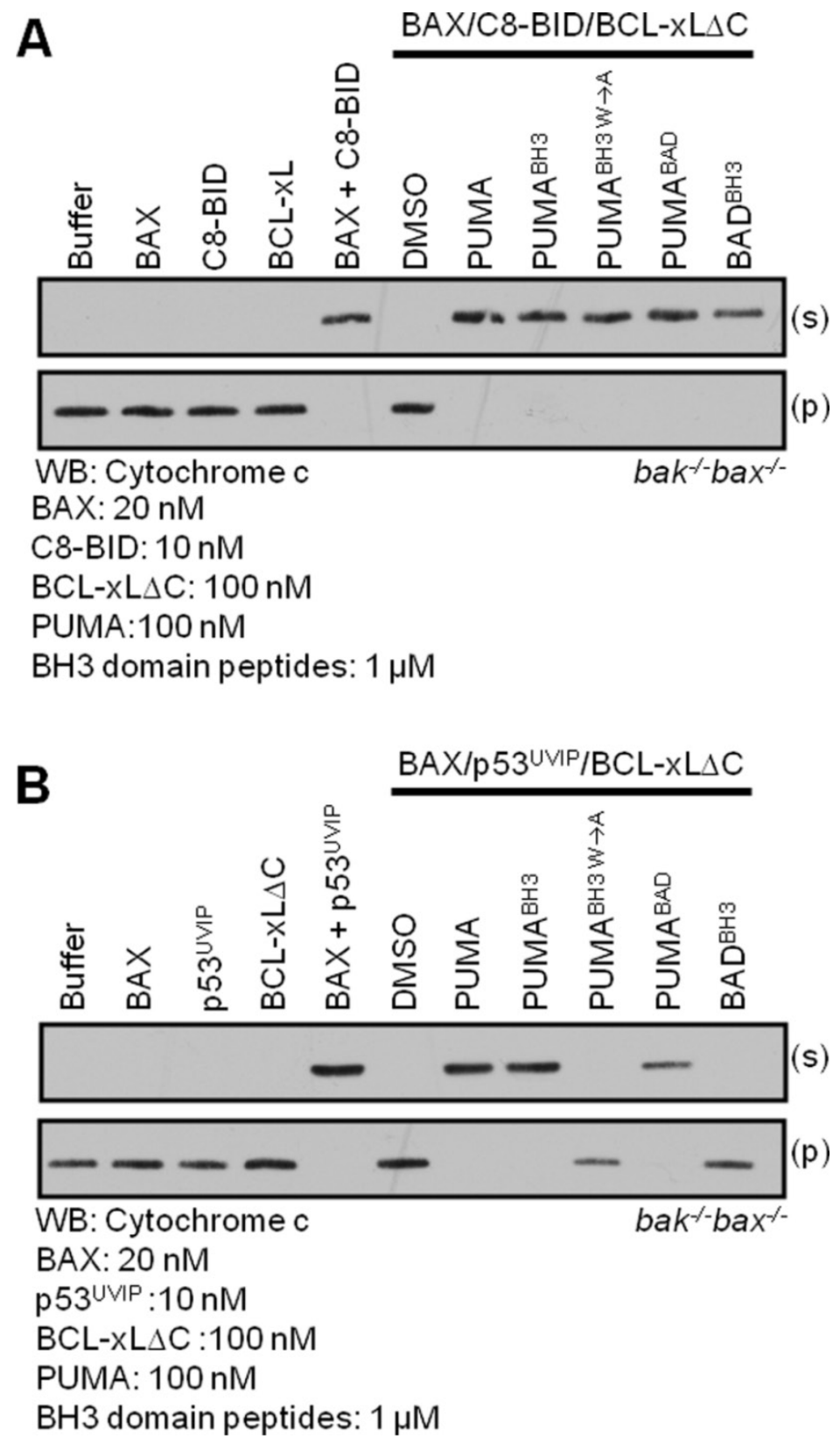

\section{Figure 3.20 PUMA W71 Is Critical for De-repression of p53 but Not C8-BID from} BCL-xL.

(A) $10 \mathrm{nM}$ complexes of BCL-xL $\Delta \mathrm{C} \cdot \mathrm{C} 8$-BID were combined with $b a k^{-/} b a x^{-/-}$liver mitochondria in the presence of BAX $(20 \mathrm{nM})$ and indicated de-repressor $\mathrm{BH} 3$ domain peptides $(1 \mu \mathrm{M})$ or PUMA $(100 \mathrm{nM})$ for one hour at $37^{\circ} \mathrm{C}$ before fractionation, SDSPAGE and western blot analyses for cytochrome c. (B) $10 \mathrm{nM}$ complexes of BCL$\mathrm{xL} \Delta \mathrm{C} \cdot \mathrm{p} 53^{\mathrm{UVIP}}$ were combined with $b a k^{-/} \mathrm{bax}^{-/-}$liver mitochondria in the presence of BAX $(20 \mathrm{nM})$ and indicated de-repressor BH3 domain peptides $(1 \mu \mathrm{M})$ or PUMA $(100 \mathrm{nM})$ for one hour at $37^{\circ} \mathrm{C}$ before fractionation, SDS-PAGE and western blot analyses for cytochrome c. 


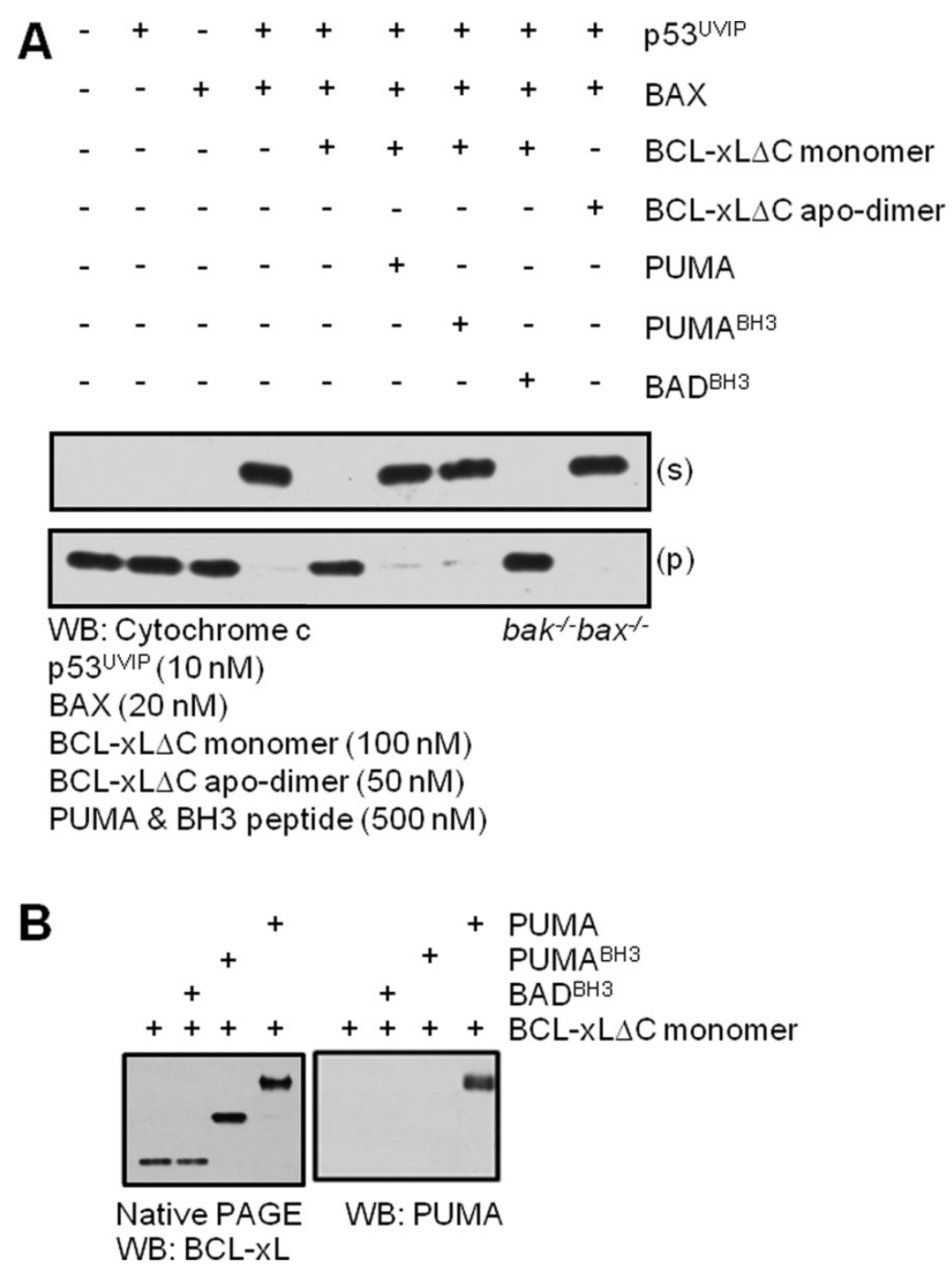

Figure 3.21 PUMA-Induced BCL-xL $\Delta C$ Dimerization Correlates with p53 ${ }^{\text {UVIP }}$ dependent BAX Activation and Subsequent Cytochrome c Release.

(A) Purified mitochondria from $b a k^{-/} b a x^{-/-}$liver were treated with the indicated proteins and/or BH3 domain peptides before fractionation, SDS-PAGE and western blot analyses for cytochrome c. (B) Supernatants from the indicated reactions in (A) were subjected to native PAGE and western blot analyses for BCL-xL $\Delta \mathrm{C}$ and PUMA. 
A

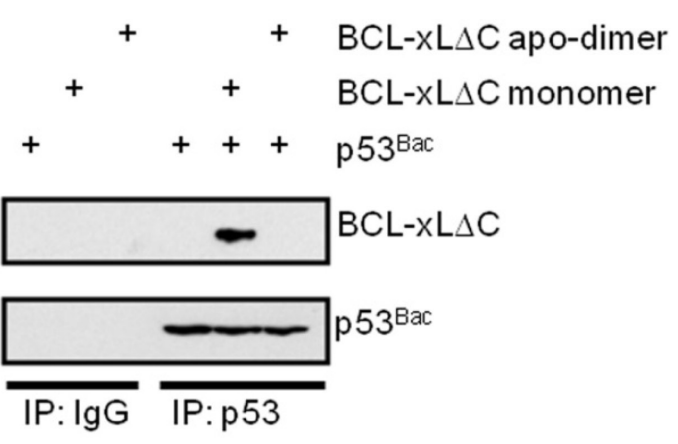

B

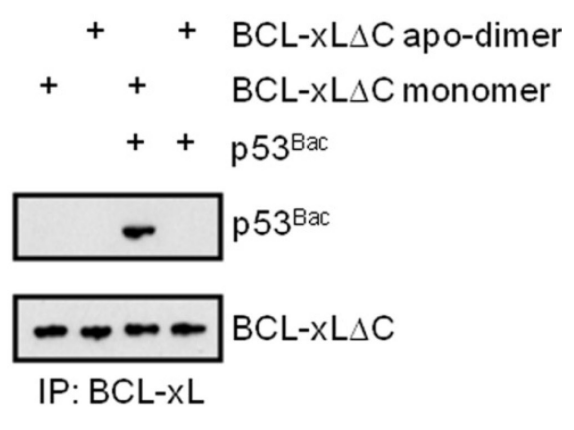

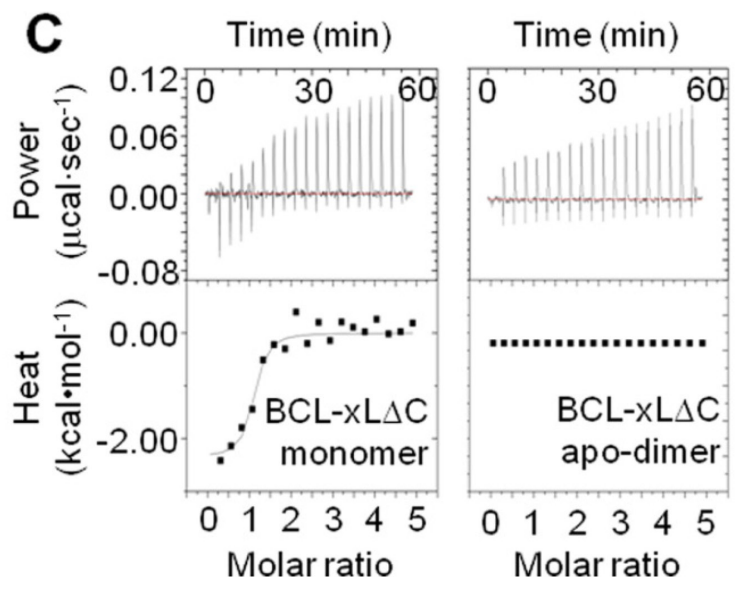

Figure 3.22 Only Monomeric BCL-xL $\Delta C$ Binds and Inhibits p53.

(A) Baculovirus-expressed p53 (100 nM) was incubated with BCL-xL $\Delta$ C monomer or BCL-xL $\Delta C$ apo-dimer $(100 \mathrm{nM})$, immunoprecipitated with antibodies against $\mathrm{p} 53^{\mathrm{UVIP}}$ (left) or BCL-xLAC (right) and subjected to SDS-PAGE and western blot analysis for $\mathrm{p} 53^{\mathrm{UVIP}}$ or BCL-xL $\Delta \mathrm{C}$. Immunoprecipitation with a mouse $\mathrm{IgG}$ was the negative control.

(B) ITC binding isotherms for BCL-xL $\Delta \mathrm{C}$ monomer and BCL-xL $\Delta \mathrm{C}$ apo-dimer $(250$ $\mu \mathrm{M})$ titrated into a solution of $\mathrm{p} 53^{\mathrm{Bac}}(10 \mu \mathrm{M})$. 
no association of BCL-xL $\Delta \mathrm{C}$ apo-dimer with $\mathrm{p} 53^{\mathrm{Bac}}$ was detected (Figure 3.22C). Together, these co-immunoprecipitation and ITC data demonstrate that BCL-xL $\Delta \mathrm{C}$ monomer and p53 directly interact with high affinity whereas BCL-xL $\Delta \mathrm{C}$ apo-dimer is unable to interact with $\mathrm{p} 53$.

We next compared the ability of BCL-xL $\Delta \mathrm{C}$ monomer and BCL-xL $\Delta \mathrm{C}$ apo-dimer to regulate p53-dependent BAX activation and MOMP. Bak $k^{--} b a x^{--}$mitochondria underwent complete cytochrome c release when treated with $\mathrm{p} 53^{\mathrm{UVIP}}$ and BAX (Figure 3.23A). This release was fully inhibited by the addition of BCL-xL $\Delta C$ monomer but not BCL-xL $\Delta C$ apo-dimer (Figure 3.23A). In contrast, both BCL-xL $\Delta C$ monomer and BCL$\mathrm{xL} \Delta \mathrm{C}$ apo-dimer inhibited C8-BID-induced cytochrome $\mathrm{c}$ release (Figure 3.23B). These reults were expected, as both BCL-xL monomer and apo-dimer possess free $\mathrm{BH} 3$ binding grooves, as demonstrated by their similar ability to bind $\mathrm{PUMA}^{\mathrm{BH} 3}$ and previous structural analyses (O'Neill et al., 2006), and therefore can sequester C8-BID to prevent MOMP. Therefore, consistent with the binding results shown in Figure 3.22, while BCL$\mathrm{xL} \Delta \mathrm{C}$ monomer can bind and inhibit cytosolic p53, BCL-xL $\Delta \mathrm{C}$ apo-dimer lacks this function Figure 3.23A. Our earlier observation that p53 was co-immunoprecipitated in a ternary complex with PUMA W71A and BCL-xL (Figure 3.16), in which BCL-xL fails to dimerize (Figure 3.17), further suggests that p53 and PUMA bind to different surfaces on BCL-xL, consistent with previous results (Petros et al., 2004a; Sot et al., 2007; Tomita et al., 2006).

We next utilized a novel cellular model system to examine the function of BCL$x L$ monomer, BCL-xL $\Delta C$ apo-dimer and BCL-xL $\Delta C$ PUMA-dimer. The breast adenocarcinoma cell line, MCF7 (which endogenously expresses wild type p53), was generated to stably express the second mitochondria-derived activator of caspases fused to green fluorescent protein (MCF7 SMAC-GFP). Following MOMP, SMAC-GFP is released from the mitochondrial intermembrane space into the cytoplasm where it is rapidly degraded by the proteasome; this allows for a high signal to noise ratio between pre- and post-MOMP detection of SMAC-GFP (Munoz-Pinedo et al., 2006). MCF7 SMAC-GFP cells exhibit punctate mitochondrial localization of SMAC-GFP, and microinjection of C8-BID (Texas Red dextran was added to all proteins to indicate microinjected cells) promoted SMAC-GFP release and degradation as shown by the loss of GFP fluorescence (Figure 3.24). C8-BID-induced MOMP was blocked by comicroinjection of either BCL-xL $\Delta \mathrm{C}$ monomer or BCL-xL $\Delta \mathrm{C}$ apo-dimer, both of which have free BH3 binding grooves (Figure 3.24).

To investigate endogenous direct activator $\mathrm{BH} 3$-only protein function, $\mathrm{MCF} 7$ SMAC-GFP cells were treated with TNF and cycloheximide, which requires C8-BID to promote MOMP and apoptosis (Rubin et al., 1988) (Figure 3.24). In this scenario, SMAC-GFP was also released and degraded, and this release was blocked by microinjection of either BCL-xL $\Delta \mathrm{C}$ monomer or BCL-xL $\Delta \mathrm{C}$ apo-dimer (Figure 3.24). This pattern of SMAC-GFP release parallels that observed for cytochrome $\mathrm{c}$ using isolated mitochondria, demonstrating that the mechanistic implications of BCL-xL dimerization are equivalent in the two types of assays. 

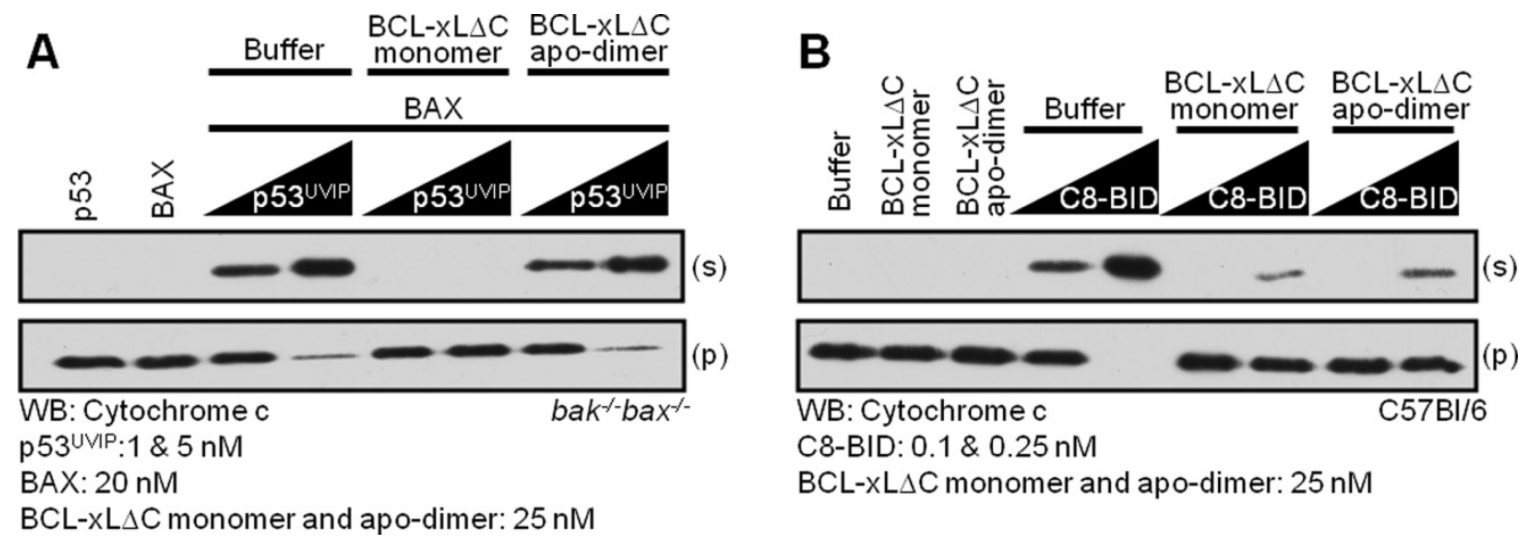

Figure 3.23 BCL-xL Monomer and BCL-xL $\Delta C$ Apo-dimer Differentially Regulate $\mathrm{p53}^{\mathrm{UVIP}}$ and C8-BID-induced Cytochrome c Release.

Purified mitochondria from $\mathrm{bak}^{-/-} \mathrm{bax}^{-/-}$(A) or C57Bl/6 (B) liver were treated with indicated proteins for one hour at $37^{\circ} \mathrm{C}$ before fractionation, SDS-PAGE and western blot analysis for cytochrome c. 


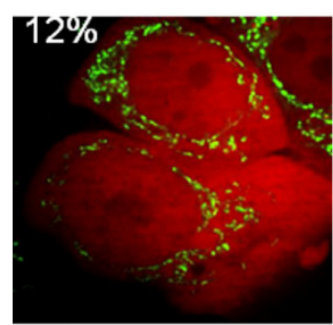

Buffer

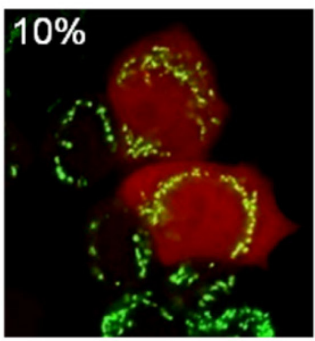

Buffer

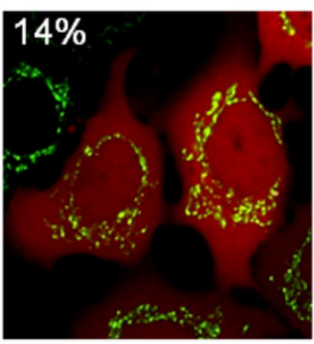

Untreated

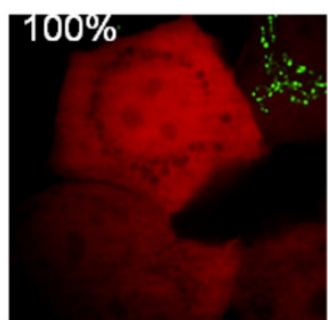

C8-BID

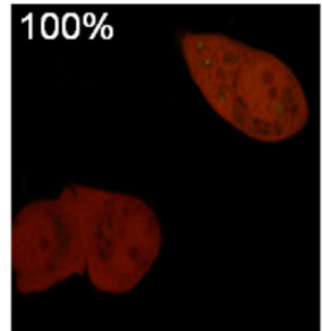

TNF/CHX

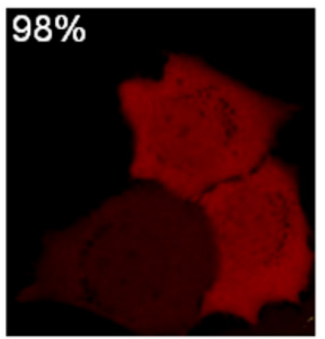

UV

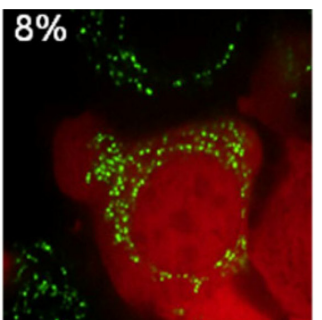

C8-BID +

BCL-xL $\Delta C$ monomer

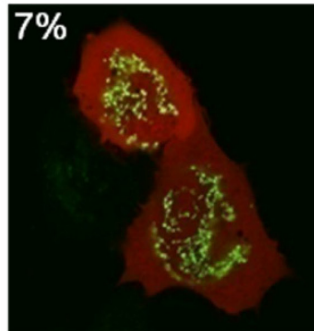

$\mathrm{TNF} / \mathrm{CHX}+$

BCL-xL $\Delta C$ monomer

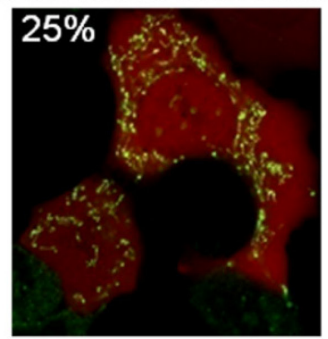

UV +

$B C L-x L \Delta C$ monomer

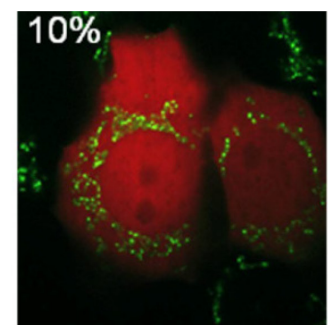

C8-BID +

BCL-xL $\Delta C$ apo-dimer

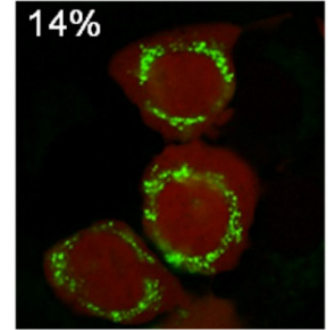

$\mathrm{TNF} / \mathrm{CHX}+$

$\mathrm{BCL}-\mathrm{LL} \Delta \mathrm{C}$ apo-dimer

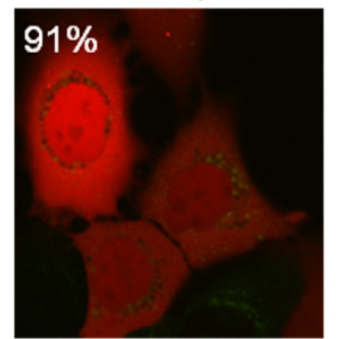

UV +

$\mathrm{BCL}-\mathrm{xL} \Delta \mathrm{C}$ apo-dimer

Figure 3.24 BCL-xL Monomer and BCL-xL $\Delta C$ Apo-dimer Differentially Regulate TNF and UV-induced MOMP.

MCF7 SMAC-GFP cells were microinjected with indicated BCL-xL $\Delta C$ variants (intracellular concentration varied from $0.47-2.35 \mathrm{nM}$ ) prior to treatment with TNF and cycloheximide $\left(2.5 \mathrm{ng} / \mathrm{ml}\right.$ and $20 \mu \mathrm{g} / \mathrm{ml}$, respectively or $40 \mathrm{~mJ} / \mathrm{cm}^{2} \mathrm{UV}$. Texas Red dextran was added to the protein solution to identify microinjected cells. Cells were imaged $5 \mathrm{~h}$ after apoptotic stimulation. MOMP was determined by the loss of SMACGFP fluorescence. The percentages of microinjected cells with a loss of SMAC-GFP fluorescence are indicated and represent results from at least 200 cells. 
The cellular implications of BCL-xL dimerization vis a vis DNA damage-induced cytosolic p53 function were investigated using MCF7 SMAC-GFP cells treated with ultraviolet (UV) radiation (Figure 3.24) UV treatment of the MCF7 SMAC-GFP cells induced MOMP as measured by the loss of SMAC-GFP fluorescence, which was inhibited by BCL-xL $\Delta \mathrm{C}$ monomer (Figure 3.24). These results paralleled those showing that dimeric forms of BCL-xL failed to bind p53 and to inhibit p53 direct activator function, suggesting strongly that UV-induced MOMP was dependent upon cytosolic $\mathrm{p} 53$. In contrast, the ability of BCL-xL to modulate $\mathrm{BH} 3$-only protein direct activator function depended not upon its oligomeric state but rather upon whether its $\mathrm{BH} 3$ binding groove was unoccupied (e.g. not bound by PUMA). 


\section{CHAPTER 4. DISCUSSION}

Our multidisciplinary studies demonstrate that PUMA induces BCL-xL dimerization via domain swapping, and that this unprecedented signaling mechanism disrupts the interaction between BCL-xL and cytosolic p53, resulting in MOMP and apoptosis. Mutation of W71 in PUMA, required for induction of BCL-xL dimerization, to alanine did not decrease the affinity of PUMA ${ }^{\mathrm{BH} 3}$ for anti-apoptotic BCL-2 family proteins including BCL-xL, MCL-1, and BFL-1 (Figure 3.14); BCL-2 was not examined in this context, due to difficulties in producing the protein for ITC measurements). Further, PUMA ${ }^{\mathrm{BH} 3}$ W71A behaved identically to PUMA in sensitizing cells for TNFinduced apoptosis, presumably because both effectively inhibited binding of antiapoptotic BCL-2 proteins to the active form of BID induced in this pathway. UVinduced expression of endogenous PUMA or ectopic expression of wild type PUMA induced dimerization of endogenous $\mathrm{BCL}-\mathrm{xL}$, which was not observed in UV-treated puma $^{-/-}$cells or upon expression of PUMA ${ }^{\mathrm{BH} 3}{ }^{\mathrm{W} 71 \mathrm{~A}}$. It was previously shown that ectopic expression of PUMA sensitizes for UV-induced apoptosis only in cells expressing p53, and that this disrupts the binding of BCL-xL to cytosolic p53 (Chipuk et al., 2005a). The finding that PUMA but not PUMA ${ }^{\mathrm{BH} 3 \mathrm{~W} \text { 11A }}$ sensitized cells for p53-dependent cell death suggested that BCL-xL dimerization and not obstruction of the BH3-binding groove is responsible for this effect. Accordingly, we found that BCL-xL apo-dimer effectively bound C8-BID and, accordingly, inhibited TNF-induced MOMP in cells; however, BCL$\mathrm{xL}$ apo-dimer failed to bind to $\mathrm{p} 53$ and did not inhibit UV-induced MOMP. Consistent with these observations, PUMA ${ }^{\mathrm{BH} 3 \mathrm{~W} \text { W1A }}$ but not PUMA co-precipitated with both BCL$\mathrm{xL}$ and $\mathrm{p} 53$. Therefore, one way in which PUMA-induced BCL-xL dimerization mediates apoptotic signaling is by affecting interactions with cytosolic p53 to control the activation of BAX; however, we do not preclude that such a dramatic structural change will affect other functions of BCL-xL.

The BCL-xL-p53 binding interface has been defined in several studies using mutagenesis, NMR chemical shift perturbation, and other biophysical techniques mapped the $\mathrm{p} 53$ binding epitope predominantly to residues that do not overlap with the canonical BH3 domain binding groove (Chipuk et al., 2004; Mihara et al., 2003; Petros et al., 2004a; Sot et al., 2007; Tomita et al., 2006) (Figure 4.1A). Based on the results of these previous studies regarding the $\mathrm{BCL}-\mathrm{xL}$ residues that interact with $\mathrm{p} 53$, we propose that BCL-xL dimerization results in burial of the p53-binding epitope (Figure 4.1B). This occlusion model, in which dimerization abolishes a portion of the surface present on BCL-xL monomers, possibly explains our observations based on ITC experiments that p53 was able to bind monomeric BCL-xL with high affinity $\left(K_{\mathrm{d}}=254 \mathrm{nM}\right)$ but was unable to bind BCL-xL apo-dimers. However, the details of the BCL-xL-p53 interaction remain elusive, as a high resolution structure for the BCL-xL-p53 complex has yet to be determined.

The specific signaling mechanism that regulates the pro-apoptotic activity of cytosolic p53 relies upon the unique ability of PUMA binding to induce BCL-xL dimerization. How does PUMA uniquely trigger BCL-xL dimerization? Past studies 

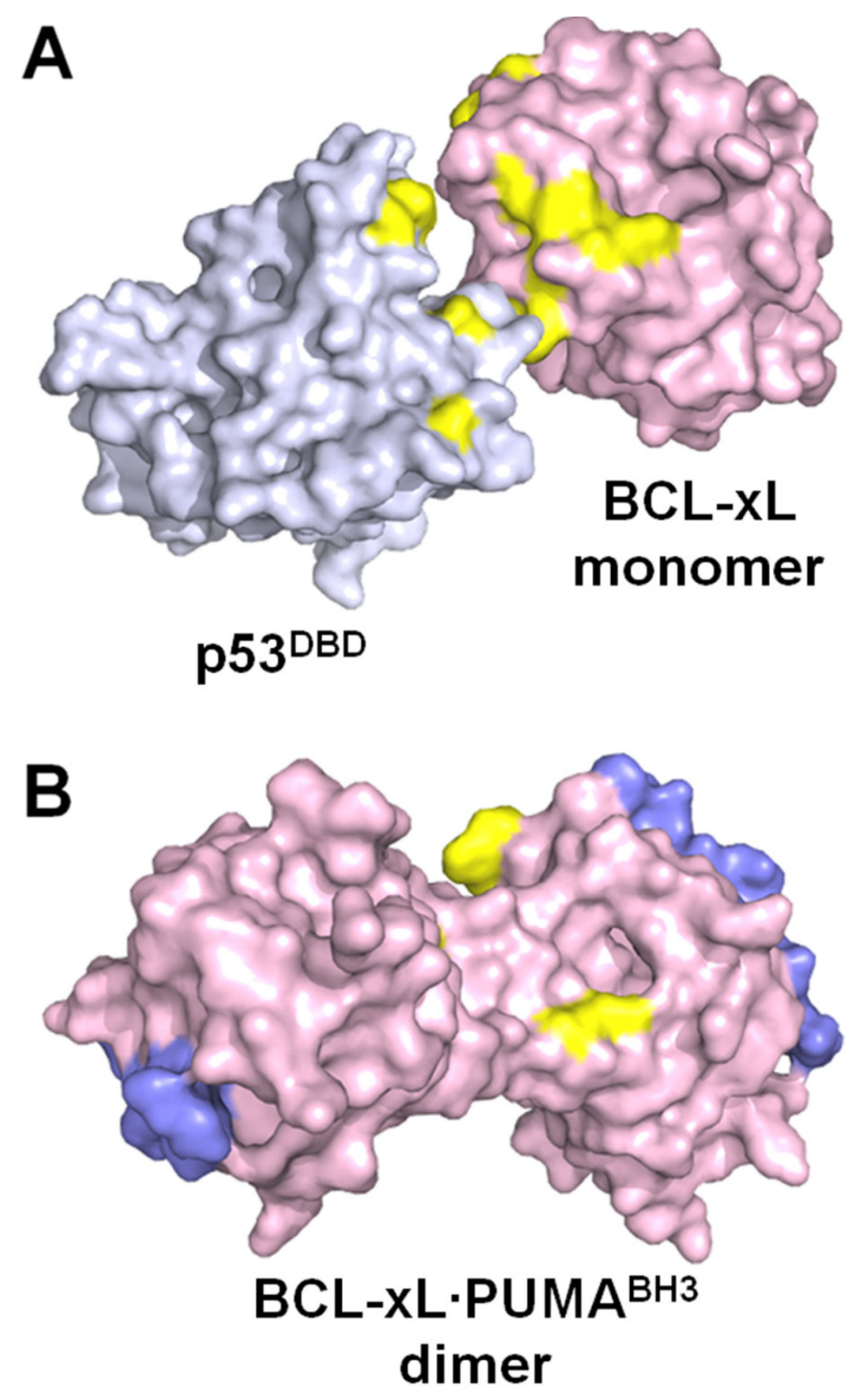

Figure 4.1 PUMA-induced Dimerization of BCL-xL Releases p53 via Burial of the p53 Binding Epitope.

(A). Residues shown by NMR chemical shift perturbation studies to be located at the BCL-xL·p53 interface are colored yellow. (B) Surface representation of the PUMA ${ }^{\mathrm{BH} 3}$ induced BCL-xL dimer. When compared with panel (A), the p53 binding surface of BCL- $x$ L is upon Dimer Formation p53 DNA binding domain $\left(\mathrm{p} 53^{\mathrm{DBD}}\right)$ is grey, BCL- $\mathrm{LL}$ is pink, and $\mathrm{PUMA}^{\mathrm{BH} 3}$ is slate. 
have shown that the structure of the BH3-binding groove of monomeric BCL-xL $\Delta \mathrm{C}$ undergoes subtle but distinct conformational changes upon binding various $\mathrm{BH} 3$ domain peptides (Liu et al., 2003). Analysis of BCL-xL·PUMA ${ }^{\mathrm{BH} 3}$ interactions within the symmetric $\mathrm{BCL}-\mathrm{xL}$ dimer provided insights into the mechanism of dimerization. A unique feature of the BCL-xL·PUMA ${ }^{\mathrm{BH} 3}$ complex discussed earlier is the $\pi$-stacking interaction between H113 of BCL-xL and W71 of PUMA, which alters the conformation of the turn connecting helices $\alpha 3$ and $\alpha 4$ relative to that observed in other BCL-xL-BH3 peptide complexes ( $\mathrm{O}^{\prime}$ Neill et al., 2006). These interactions are enabled by other notable structural changes which occur within $\alpha 3$, which comprises 3 helical turns in the BCL$\mathrm{xL} \Delta \mathrm{C}$ monomer. $\mathrm{PUMA}^{\mathrm{BH} 3}$ binding-induced extension of the $\mathrm{C}$-terminal end of $\alpha 2$ and melting of the three helical turns of $\alpha 3$ leaving a single helical turn which optimally positions BCL-xL residue $\mathrm{H} 113$ for $\pi$-stacking with $\mathrm{W} 71$ of the $\mathrm{PUMA}^{\mathrm{BH} 3}$. Binding of $\mathrm{PUMA}^{\mathrm{BH} 3}$ also caused the N-terminal region of BCL-xL $\alpha 4$ to translate toward the bound $\mathrm{BH} 3$ ligand to facilitate protein-ligand interactions, whereas the C-terminal end of $\alpha 4$ pivoted outward, widening the $\mathrm{BH} 3$ domain binding groove. We propose that similar binding-induced structural rearrangements, mediated partly by the BCL-xL H113.PUMA W71 interaction that is unique among BCL-xL-BH3 domain peptide complexes, occur when PUMA binds to monomeric BCL-xL and that they transiently trigger disengagement of helices $\alpha 6-\alpha 8$ from the $\alpha 1-\alpha 5$ helical BC core and ultimately BCL-xL dimerization.

It has previously been reported that several $\mathrm{BH} 3$-only proteins (e.g., BAD, BIM, and BMF) are IDPs (Hinds et al., 2006). Using CD and NMR spectroscopy we similarly found that full-length human PUMA is an IUP, devoid of tertiary or quaternary structure. Far-UV CD spectropolarimetry revealed nascent helicity within the PUMA polypeptide that was localized within the BH3 domain. Interestingly, the amount of nascent helical secondary structure present in full-length PUMA is nearly identical to that observed for the 25 amino acid PUMA ${ }^{\mathrm{BH} 3}$, suggesting that sequences flanking the PUMA BH3 domain do not significantly stabilize BH3 domain helicity through helical end effects. Many IUPs function by folding upon binding their biological targets with the dynamic and largely disordered free state often exhibiting partially populated structural features that are observed to be fully populated in the bound-state (e.g., p2 $7^{\text {Kipl }}$ ) (Lacy et al., 2004; Sivakolundu et al., 2005).

The nascent $\alpha$-helix observed within the BH3 domain of PUMA is induced to fully fold upon binding to the hydrophobic groove of BCL-xL $\Delta$ C). The PUMA BH3 domain adopts generally similar conformations when bound to BCL-2 family proteins, including BCL-xL (as shown herein), MCL-1 (Day et al., 2008) and BFL-1 (Smits et al., 2008). However, while many interactions between PUMA and these highly related proteins are similar among these three complexes, we noted several differences. For example, Y90 of PUMA packs in hydrophobic pockets of MCL-1 and BFL-1 in the same conformation relative to the axis of the PUMA ${ }^{\mathrm{BH} 3} \alpha$-helix (Figure 4.2). In contrast, Y90 packs into a pocket on the opposite side of the $\mathrm{BH} 3$ binding groove in the BCL$\mathrm{xL} \triangle \mathrm{C} \cdot \mathrm{PUMA}^{\mathrm{BH} 3}$ complex. Similar binding promiscuity is observed for $\mathrm{W} 71$ of the $\mathrm{PUMA}^{\mathrm{BH} 3}$ that, upon binding to $\mathrm{BCL}-\mathrm{xL}$, is critical for BCL-xL dimerization and release of p53. This residue exhibits the same conformation and limited interactions with polar 


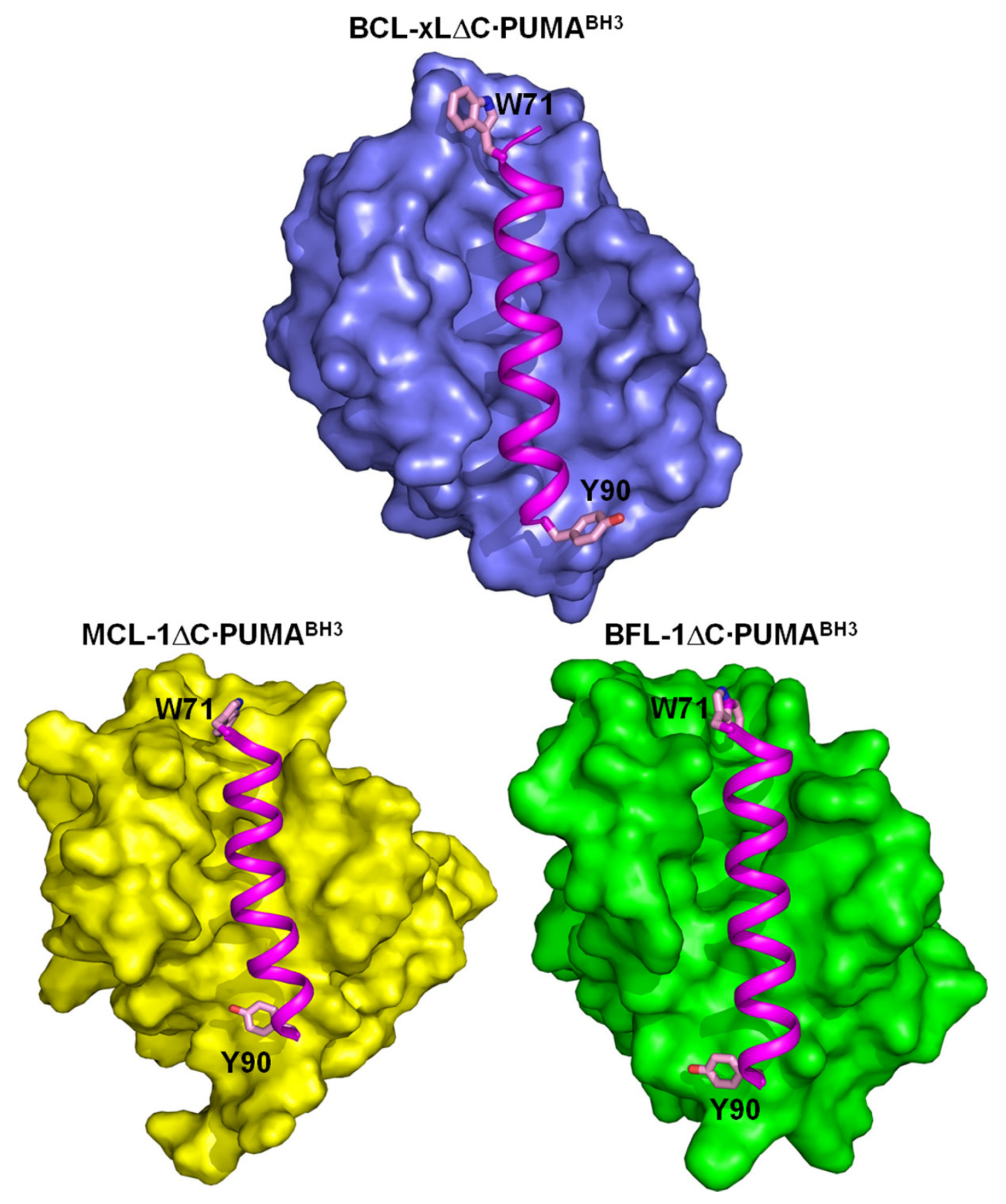

Figure 4.2 Structural Comparison of BCL-xL $\Delta C \cdot P U M A^{B H 3}$, MCL$1 \Delta C \cdot P U M A A^{B H 3}$, and BFL-1 $\Delta C \cdot P_{U M A}{ }^{B H 3}$ Complexes.

W71 and Y90 side chains (shown in black) of PUMA ${ }^{\mathrm{BH} 3}$ showed drastically different positions within the BCL-xL·PUMA ${ }^{\mathrm{BH} 3}$, MCL-1·PUMA ${ }^{\mathrm{BH} 3}$ (PDB ID:2ROC) complexes., and BFL-1.PUMA ${ }^{\mathrm{BH} 3}$ (PDB ID:2VOF) complexes. BCL-xL, MCL-1, and BFL-1 are slate, yellow, and green, respectively. $\mathrm{PUMA}^{\mathrm{BH} 3}$ is magenta. 
and hydrophobic moieties on the surfaces of MCL-1 and BFL-1 (Figure 4.2). However, through rotation of the $\chi_{1}$ torsion angle, W71 interacts favorably with BCL-xL $\Delta$ C H113 through $\pi$-stacking interactions (Figure 4.2).

IDPs often interact with their targets through short, linear motifs (Fuxreiter et al., 2007; Vacic et al., 2007). For PUMA, a protein of 193 residues ( $\alpha$ isoform) or 131 residues ( $\beta$ isoform), the 25 residue $\mathrm{BH} 3$ domain peptide appears to be necessary and sufficient for regulation of the BCL-2 protein family. The intrinsic flexibility of this BH3 domain provides the potential for adaptive binding in distinct conformations depending upon the structural context (e.g., binding to BCL-xL, BFL-1 or MCL-1) which can produce dramatically different biological outcomes. This structural plasticity allows PUMA to play multiple roles in regulating BCL-2 family proteins. First, PUMA binds tightly to the $\mathrm{BH} 3$ binding grooves of the entire BCL-2 repertoire (Chen et al., 2005), displacing more weakly bound $\mathrm{BH} 3$-only proteins, including the direct activators, BID and BIM; this function enables PUMA to broadly regulate apoptosis through mechanisms termed de-repression/sensitization, described above in Chapter 1. Second, PUMA, through the unique, binding-induced structural changes discussed above, induces BCL$\mathrm{xL}$ dimerization; this function specifically mediates UV-induced, p53-dependent MOMP and apoptosis. It is fascinating that, while the PUMA BH3 domain conserves many sequence features common to $\mathrm{BH} 3$ domains of other $\mathrm{BH} 3$-only proteins, amino acid differences at just a few positions within this domain (e.g., position 71) enable PUMA to function as both a general sensitizer and de-repressor of direct activator $\mathrm{BH} 3$-only proteins and a specific modulator of cytosolic p53-dependent apoptosis.

Protein oligomerization via domain swapping is one means by which highly efficient protein interaction networks have evolved (Liu and Eisenberg, 2002). The physiological relevance of dimerization via domain-swapping has been shown in proteins such as diphtheria toxin, which undergoes ligand-induced dimerization via domain swapping upon binding its receptor at physiologic $\mathrm{pH}$ (Louie et al., 1997). Interestingly, the structures of BCL-xL and diphtheria toxin are strikingly similar despite limited sequence homology (Muchmore et al., 1996b). Regarding BCL-xL dimerization via domain swapping, there is a substantial energy difference between the "closed" and "open" monomeric forms (e.g., with helices $\alpha 6-\alpha 8$ dislodged from the $\alpha 1-\alpha 5$ core), which constitutes an energy barrier for BCL-xL dimerization due to the need to disrupt many favorable packing interactions to form the "open" conformer (Liu and Eisenberg, 2002). Once generated, two "open" BCL-xL monomers interact to form the domainswapped dimer. This energy barrier can be reduced by exposure of BCL-xL to nonphysiological extremes of pH (O'Neill et al., 2006) or heat (Denisov et al., 2007a).

In the present study, we identified a means by which BCL-xL domain swap dimerization occurs under physiologically relevant conditions as a result of PUMA or $\mathrm{PUMA}^{\mathrm{BH} 3}$ binding. We propose that alteration of hydrophobic packing between the $\alpha 3$ $\alpha 4$ and $\alpha 5-\alpha 6$ "layers" of monomeric BCL-xL due to specific structural rearrangements imposed by PUMA binding, in which the BCL-xL H113·PUMA W71 interaction discussed above is critical, decreases the energy barrier, triggering transient opening and dimerization. We have observed that $\mathrm{PUMA}^{\mathrm{BH} 3}$-induced dimerization does not exhibit a 
dynamic, reversible equilibrium. This implies that the energy barrier for the reverse process (dimer to monomer) is large, most likely due to the favorability of the new interface which is formed upon rearrangement of the strained reverse turn in the BCL-xL monomer (O'Neill et al., 2006) into two side by side anti-parallel helices in the $\mathrm{PUMA}^{\mathrm{BH} 3}$-induced $\mathrm{BCL}-\mathrm{xL}$ dimer. The dead-end nature of this signaling mechanism, with PUMA triggering irreversible $\mathrm{BCL}-\mathrm{xL}$ dimerization, is compatible with the biological outcome of the signal itself: to trigger MOMP following DNA damage and ultimately cell death. While the exact mechanism(s) of BAK and BAX oligomerization have yet to be resolved, our data for BCL-xL dimerization by domain swapping raises the interesting possibility that domain swapping might play a role in the formation BAK and/or BAX oligomers following direct activator stimulation, as previously hypothesized (Leber et al., 2007).

The failure of PUMA ${ }^{\mathrm{BH} 3 \mathrm{~W} 71 \mathrm{~A}}$ to promote $\mathrm{p} 53$-dependent apoptosis might be due to an effect of this substitution on the affinity of PUMA for anti-apoptotic BCL-2 proteins. However, we found that mutation of W71 did not affect affinity for BCL-xL, and slightly increased affinity for MCL-1 and BFL-1. The ability of PUMA ${ }^{\mathrm{BH} 3 \mathrm{~W} 71 \mathrm{~A}}$ to promote TNF-induced apoptosis (which is p53 independent) and of the $\mathrm{PUMA}^{\mathrm{BH} 3 \mathrm{~W} 71 \mathrm{~A}}$ and BAD-PUMA ${ }^{\mathrm{BH} 3}$ peptides to disrupt the BCL-x $\triangle \mathrm{C} \cdot \mathrm{C} 8$-BID complex to promote MOMP is similarly inconsistent with a general defect in the de-repressor activity of PUMA $^{\text {BH3 W71A. }}$

Unlike BCL-xL, neither BCL-2 nor MCL-1 undergoes dimerization upon interaction with PUMA. Both BCL-2 (Tomita et al., 2006) and MCL-1 (Leu et al., 2004) reportedly bind to cytosolic $\mathrm{p} 53$, but the affinities and regulation of these interactions versus those with BCL-xL are not yet known. In cells lacking PUMA (e.g. puma ${ }^{-/-}$MEFs and HCT116 PUMA $A^{-/}$cells), immunoprecipitation of BCL-xL following DNA damage removed all available cytosolic p53 (Chipuk et al., 2005b), and therefore the regulation of p53 by PUMA-induced dimerization of BCL-xL is likely to be physiologically important. However, the situation is potentially more complex, as the interaction of PUMA with all of the anti-apoptotic BCL-2 proteins prevents their association with other pro-apoptotic BCL-2 proteins (Chen et al., 2005; Uren et al., 2007; Willis et al., 2007). Further analysis comparing the anti-apoptotic effects of BCL-xL versus BCL-2 and MCL-1 in the context of p53-dependent apoptosis will continue to reveal the mechanisms of action for the BCL-2 family and MOMP.

\section{A PUMA BH3 Mimetic for Cancer Treatment}

Cancer research worldwide has one principal objective: to find a cure for cancer. As overly simplistic and obvious as this goal may at first sound, being that each neoplastic subtype is born forth from an amalgam of spontaneous random gene mutations that by pure chance allow for clonal selection, numerous studies within the last decade have unveiled a phenomenon based on principles known for years. Most, if not all, cancers likely have an Achilles' heel (Green and Evan, 2002) (Letai, 2008). Ironically, this Achilles' heel is based on the tenants of cell death. 
The BCL-2 family is implicated in a vast array of diseases, although the best characterized BCL-2 family related malady to date is arguably cancer. Great effort has been made to generate pharmacological regulators of the BCL-2 family for clinical use as single-agent or combination anti-cancer therapies. The most potent and prevalent mechanism of apoptosis dysregulation in cancer is overexpression of anti-apoptotic family members (Reed, 1997; Wei, 2004). Examples of increased BCL-2 or BCL-xL levels in human malignancy include leukemias, lymphomas, and solid tumor epithelial malignancies (Letai, 2008). Over-expression of anti-apoptotic proteins has been shown to be directly correlated with neoplastic aggressiveness, poor prognosis, and chemotherapeutic resistance (Wei, 2004).

\section{Experimental Rationale}

An emerging theme in cancer biology is that tumor cells become addicted to high levels of anti-apoptotic BCL-2 family proteins in order to maintain survival. This theme is in direct agreement with the landmark review "The Hallmarks of Cancer" (Hanahan and Weinberg, 2000). One requirement for cancer cells is acquisition of autonomy from normal mitogenic signals such that cells continuously divide in an uncontrolled, chaotic manner. This is achieved predominantly by constitutive activation of oncogenes such as $R a s, E 1 A, M y c$, and $E 2 F$, which, under normal, non-pathological circumstances trigger apoptotic cell death (Evan and Littlewood, 1998; Harrington et al., 1994). Accordingly, inhibiting the anti-apoptotic proteins in tumors should induce spontaneous apoptosis of the tumor cells or marked sensitization to pro-apoptotic BCL-2 family members which, as a result of constitutive oncogenic signaling, induce direct activator $\mathrm{BH} 3$-only proteins, as seen in chronic lymphocytic leukemia where the neoplastic cells' anti-apoptotic repertoires are "primed" by BIM . Oncogenic priming of tumor cells with direct activator $\mathrm{BH} 3-$ only proteins provides a therapeutic window for the use of small molecule, derepressor $\mathrm{BH} 3$-only protein mimetics for cancer treatment, as most normal, healthy cells do not exhibit direct activator $\mathrm{BH} 3$-only protein activation and thus are not primed for death.

Following crystal and NMR structure determination of BCL-xL (Muchmore et al., 1996a), several drugs were designed to target BCL-xL and the other anti-apoptotic BCL2 proteins (e.g., ABT-737) (Oltersdorf et al., 2005). A comparison of several inhibitors of the anti-apoptotic BCL-2 proteins showed that only ABT-737 (Abbot Laboratories), discovered by obtaining structure-activity relationships by NMR (SAR by NMR) (Hajduk et al., 1997), caused cell death in a BAK/BAX-dependent manner (van Delft et al., 2006). This small molecule inhibitor neutralizes BCL-2, BCL-xL, and BCL-w with subnanomolar affinity and exhibits marked cytotoxic efficacy in combination with chemotherapeutics and radiation, although healthy primary cells are not killed (Oltersdorf et al., 2005). In pre-clinical studies, ABT-263, an orally bioavailable derivative of ABT737 , displayed single-agent effectiveness against a variety of tumor types including lymphoma and small cell lung cancer (Oltersdorf et al., 2005). 
Overexpression of MCL-1 has been shown to be a potent mechanism of resistance to ABT-737 and ABT-263, as these small molecule BH3-only protein mimetics have a binding profile identical to that of BAD, and therefore do not bind MCL-1 (Certo et al., 2006; Kuwana et al., 2005) (Figure 1.4A). Cancer cells that express high levels of MCL1 , therefore, are refractory to treatment with the current ABT-737-like compounds.

The structure of the BCL-xL·PUMA ${ }^{\mathrm{BH} 3}$ complex we have solved in combination with the recently solved structures of MCL-1 (Day et al., 2005), MCL-1·BIM ${ }^{\mathrm{BH} 3}$ (Czabotar et al., 2007), and MCL-1·PUMA ${ }^{\mathrm{BH} 3}$ (Day et al., 2008) provide a wealth of knowledge regarding the intricacies of $\mathrm{BH} 3$-domain peptide-anti-apoptotic protein interactions. This wealth of knowledge can be used to design novel small molecule $\mathrm{BH} 3$ domain peptide mimetics that could possibly overcome the resistance of some cancer cells to ABT-737-like compounds caused by increased MCL-1 expression.

ABT-737 has been used as a starting small molecule scaffold to develop a pan inhibitor of the anti-apoptotic repertoire, although to date these studies have shown no success (Fu et al., 2007; Lee et al., 2009). These studies highlight the differences in structural properties the anti-apoptotic protein repertoire. These differences are likely to be extremely important in developing a pan-inhibitor of the anti-apoptotic repertoire. Indeed, it was concluded that flexibility of both the small molecule BH3 mimetic and the anti-apoptotic protein significantly contribute to the ability of rationally designed variants of $\mathrm{ABT}-737$ derivatized to mimic $\mathrm{BIM}^{\mathrm{BH} 3}$ to retain binding to $\mathrm{BCL}-\mathrm{xL}$ (which exhibits a high degree of flexibility within the $\mathrm{BH} 3$ domain binding groove) but still failed to bind MCL-1 (which shows no flexibility within the BH3 binding groove due to the complete retention of helicity in helix 3 of the groove) (Lee et al., 2009).

\section{Proposal and Experimental Design}

Our structure of the BCL-xL·PUMA ${ }^{\mathrm{BH} 3}$ complex described above may provide an alternative route to accomplishing the goal of developing a novel ABT-737 like compound that may not bind MCL-1 but still circumvent the cancer cell resistance afforded by MCL-1 overexpression. Using ABT-737 as a model to produce a derivative that retains the activity of this small molecule (de-repression of BIM and/or BID primed anti-apoptotic proteins) but targets a slightly different area within the BCL-xL BH3binding groove to mimic the novel $\pi$-stacking interaction seen within our structure would result in a $\mathrm{BH} 3$ domain peptide mimetic that would also induce BCL-xL dimerization and de-repression of an additional direct activator, cytosolic p53.

For development of such a compound, the principles of SAR by NMR (Hajduk et al., 1997) would be employed. Assignments for BCL-xL monomer are available as well as assignments for the BCL-xL apo-dimer (Denisov et al., 2007a). First, a library of small molecule fragments would be designed to encompass a wealth of small molecule fragments containing at least one aromatic group requisite to recapitulate the $\pi$-stacking feature of W71 in PUMA ${ }^{\mathrm{BH} 3}$, which we have shown is necessary for PUMA-induced BCL-xL dimerization (Figure 3.12 and Figure 3.13) and p53 release from BCL-xL 
(Figure 3.16). This aromatic group-based small molecule fragment library would be used in 2D TROSY NMR experiments wherein the fragments would be added to ${ }^{15} \mathrm{~N}$-labeled BCL-xL $\Delta C$ and the chemical shifts of BCL-xL H113 and residues adjacent to it would be monitored for perturbation (Figure 3.28). In the fragment screening process, it would be critical to screen the small molecules individually or in small mixtures to avoid fragment aggregation resulting from hydrophobic or $\pi$-stacking interactions. Fragment solubility would also be a concern; therefore the library would need to include aromatic rings that are derivatized to increase solubility (e.g., aromatic ring systems with various polar or hydrophilic groups attached).

In addition to screening for small molecule fragments capable of engaging in a $\pi$ stacking interaction with ${ }^{15} \mathrm{~N}$-labeled BCL-xL $\Delta \mathrm{C}$, it would also be necessary to screen for additional small molecule fragments capable of specifically binding within the adjacent hydrophobic pockets that surround PUMA I75 and the conserved L79 (formed by BCL$\mathrm{xL} \Delta \mathrm{C}$ residues V126, L1 12, L108, F146, F105 and F146, F105, V126, F97, L130 and A142, respectively) using NMR as described above (Figure 4.3). In ABT-737, a chlorbiphenyl moiety engages the PUMA L79 pocket, and could therefore be a high likelihood candidate (Lee et al., 2007) (Figure 4.3). Finding small molecule fragments capable of packing specifically into these hydrophobic pockets is crucial, as they would provide the crucial building blocks to use to link any aromatic small molecule fragment mimetic of PUMA W71.

Positive hits from the initial NMR screen that show chemical shift perturbation in the $\mathrm{BCl}-\mathrm{xL} \mathrm{H} 113$ vicinity would then be subjected to a further screen wherein each is tested for its ability to induce BCL-xL $\Delta \mathrm{C}$ in native page dimerization assay. Because small molecule fragments normally have relatively low affinity for their target protein (Sattler et al., 1997), millimolar concentrations of each positive hit fragment would be added to a small volume of approximately $25 \mu \mathrm{M}$ BCL-xL $\Delta \mathrm{C}$, incubated at $30^{\circ} \mathrm{C}$ for one hour, and the resulting treatments would be screened for BCL-xL $\Delta \mathrm{C}$ dimerization by native PAGE.

Fragments that induce BCL-xL $\Delta \mathrm{C}$ dimerization would then be linked using synthetic chemistry approaches to connect small molecule fragments found to engage PUMA 175 and L79 within the BH3 binding groove. The approach to linking these small fragments would be based on the surface topology characteristics between these binding pockets so as to avoid issues such as steric clashes between the compound and BCL-xL side chains as well as attempting to avoid charge-charge incompatibility between the backbone linker scaffold and BCL-xL. Linker scaffolds should also afford a degree of flexibility in order for the final PUMA small molecule mimetic to engage the $\mathrm{BH} 3$ domain binding groove in the manner seen for $\mathrm{PUMA}^{\mathrm{BH} 3}$, which folds upon binding and therefore results in optimal positioning of peptide-protein interactions within the BCL-xL binding groove. Additionally, BCL-xL refolds to accommodate the incoming peptide or 


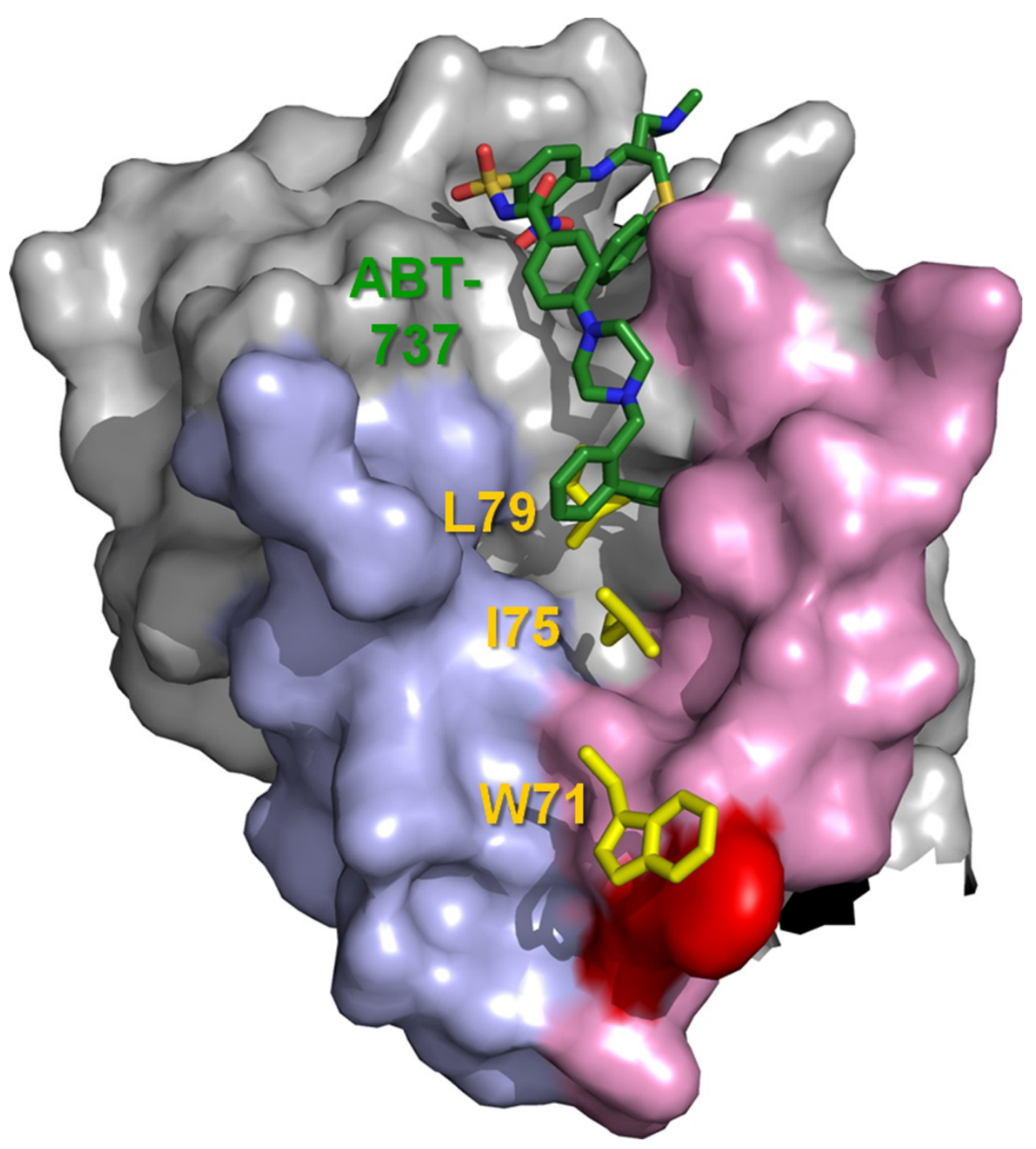

Figure 4.3 Target Areas of BCL-xL Used to Screen for a Novel Small Molecule PUMA $^{\text {BH3 }}$ Mimetic.

Surface representation of one side of the BCL-xL PUMA ${ }^{\mathrm{BH} 3}$ dimer complex is shown for clarity. Helix 3 is pink and helix four is blue. The three primary small fragment target sites for structure activity relationship by nuclear magnetic resonance (SAR by NMR) are adjeacent to the PUMA ${ }^{\mathrm{BH} 3}$ side chains in yellow. ABT-737 (green) was superposed using the BCL-xL $\Delta \mathrm{L} \Delta \mathrm{C} \cdot \mathrm{ABT}-737$ structure (PDB ID:2YXJ). PUMA L79 and the chlorbiphenyl moiety of ABT-737 overlap and occupy the same hydrophobic binding pocket. 
BH3 mimetic compound (specifically helix 3 described above) (Lee et al., 2009). If a PUMA BH3 mimetic capable of inducing BCL-xL dimerization is successfully accomplished, the compound would likely need to undergo further chemical modifications in order modulate its solubility and pharmacokinetics to ensure it is able to not only make itto the cancer cells (which can be impeded by binding of compounds to serum proteins which reduces bioavailability) as well as penetrate the tumor cells (Oltersdorf et al., 2005).

A PUMA mimetic would likely show the highest efficacy in cancer types that retain the ability to upregulate wild type p53. In this scenario, cancer cells primed for death by the direct activator BH3-only proteins BID and/or BIM and/or by cytosolic p53 (induced by continuous oncogenic signaling as described above), would undergo MOMP and cell death via de-repression by the PUMA BH3 mimetic. Cytosolic p53 has been found in numerous tumor cell lines bound to BCL-xL and engages MOMP in a PUMAdependent manner (Speidel et al., 2006). A BH3 mimetic able to de-repress not only two but three different direct activator proteins should increase the likelihood of cancer cell death.

It is interesting to speculate that such a novel BH3 mimetic could indeed show binding to MCL-1. Unlike ABT-737 which engages the $\mathrm{BH} 3$ binding groove near the proteins C-terminus (Lee et al., 2007), a PUMA mimetic designed as described above would target the opposite end of the binding groove. It is possible that targeting this region of MCL-1 might allow higher affinity binding since the hydrophobic pockets used to engage $\mathrm{BH} 3$-only proteins are deeper within this region, whereas the pockets targeted by ABT-737 are much shallower (Day et al., 2005) and cause steric clash. If the PUMA mimetic does show appreciable binding to MCL-1, cancers resistant to ABT-737-like compounds would likely be susceptible to the novel PUMA mimetic, independent of its ability to de-repress cytosolic p53. 


\section{LIST OF REFERENCES}

Akiyama, T., Bouillet, P., Miyazaki, T., Kadono, Y., Chikuda, H., Chung, U.I., Fukuda, A., Hikita, A., Seto, H., Okada, T., et al. (2003). Regulation of osteoclast apoptosis by ubiquitylation of proapoptotic BH3-only Bcl-2 family member Bim. EMBO J 22, 66536664.

Amundson, S.A., Myers, T.G., Scudiero, D., Kitada, S., Reed, J.C., and Fornace, A.J., Jr. (2000). An informatics approach identifying markers of chemosensitivity in human cancer cell lines. Cancer Res 60, 6101-6110.

Ashcroft, M., Taya, Y., and Vousden, K.H. (2000). Stress signals utilize multiple pathways to stabilize p53. Mol cell biol 20, 3224-3233.

Bairoch, A., Apweiler, R., Wu, C.H., Barker, W.C., Boeckmann, B., Ferro, S., Gasteiger, E., Huang, H., Lopez, R., Magrane, M., et al. (2005). The Universal Protein Resource (UniProt). Nucleic Acids Res 33, D154-159.

Balbo, A., Brown, P.H., Braswell, E.H., and Schuck, P. (2007). Measuring proteinprotein interactions by equilibrium sedimentation. Curr Protoc Immunol Chapter 18, Unit 1818.

Beroukhim, R., Mermel, C.H., Porter, D., Wei, G., Raychaudhuri, S., Donovan, J., Barretina, J., Boehm, J.S., Dobson, J., Urashima, M., et al. (2010). The landscape of somatic copy-number alteration across human cancers. Nature 463, 899-905.

Bevers, E.M., and Williamson, P.L. (2010). Phospholipid scramblase: an update. FEBS Lett 584, 2724-2730.

Boatright, K.M., Renatus, M., Scott, F.L., Sperandio, S., Shin, H., Pedersen, I.M., Ricci, J.E., Edris, W.A., Sutherlin, D.P., Green, D.R., et al. (2003). A unified model for apical caspase activation. Mol Cell 11, 529-541.

Braithwaite, A.W., Royds, J.A., and Jackson, P. (2005). The p53 story: layers of complexity. Carcinogenesis 26, 1161-1169.

Brunger, A.T., Adams, P.D., Clore, G.M., DeLano, W.L., Gros, P., Grosse-Kunstleve, R.W., Jiang, J.S., Kuszewski, J., Nilges, M., Pannu, N.S., et al. (1998). Crystallography \& NMR system: A new software suite for macromolecular structure determination. Acta Crystallogr D Biol Crystallogr 54, 905-921.

Bullock, A.N., and Fersht, A.R. (2001). Rescuing the function of mutant p53. Nat Rev Cancer 1, 68-76.

Caelles, C., Heimberg, A., and Karin, M. (1993). p53-Dependent apoptosis in the absence of transcriptional activation of p53-target genes. Nature 370, 220-223. 
Certo, M., Moore Vdel, G., Nishino, M., Wei, G., Korsmeyer, S., Armstrong, S.A., and Letai, A. (2006). Mitochondria primed by death signals determine cellular addiction to antiapoptotic BCL-2 family members. Cancer Cell 9, 351-365.

Chen, L., Willis, S.N., Wei, A., Smith, B.J., Fletcher, J.I., Hinds, M.G., Colman, P.M., Day, C.L., Adams, J.M., and Huang, D.C. (2005). Differential targeting of prosurvival $\mathrm{Bcl}-2$ proteins by their BH3-only ligands allows complementary apoptotic function. Mol Cell 17, 393-403.

Chipuk, J.E., Bouchier-Hayes, L., Kuwana, T., Newmeyer, D.D., and Green, D.R. (2005a). PUMA couples the nuclear and cytoplasmic proapoptotic function of $\mathrm{p} 53$. Science 309, 1732-1735.

Chipuk, J.E., Bouchier-Hayes, L., Kuwana, T., Newmeyer, D.D., and Green, D.R. (2005b). PUMA couples the nuclear and cytoplasmic proapoptotic function of p53. Science 309, 1732-1735.

Chipuk, J.E., Fisher, J.C., Dillon, C.P., Kriwacki, R.W., Kuwana, T., and Green, D.R. (2008). Mechanism of apoptosis induction by inhibition of the anti-apoptotic BCL-2 proteins. Proc Natl Acad Sci U S A 105, 20327-20332.

Chipuk, J.E., and Green, D.R. (2005). Do inducers of apoptosis trigger caspaseindependent cell death? Nat Rev Mol Cell Biol 6, 268-275.

Chipuk, J.E., and Green, D.R. (2008). How do BCL-2 proteins induce mitochondrial outer membrane permeabilization? Trends Cell Biol 18, 157-164.

Chipuk, J.E., Kuwana, T., Bouchier-Hayes, L., Droin, N.M., Newmeyer, D.D., Schuler, M., and Green, D.R. (2004). Direct activation of Bax by p53 mediates mitochondrial membrane permeabilization and apoptosis. Science 303, 1010-1014.

Chipuk, J.E., Maurer, U., Green, D.R., and Schuler, M. (2003). Pharmacologic activation of p53 elicits Bax-dependent apoptosis in the absence of transcription. Cancer Cell 4, 371-381.

Chipuk, J.E., Moldoveanu, T., Llambi, F., Parsons, M.J., and Green, D.R. (2010). The BCL-2 family reunion. Mol Cell 37, 299-310.

Chou, J.J., Li, H., Salvesen, G.S., Yuan, J., and Wagner, G. (1999). Solution structure of BID, an intracellular amplifier of apoptotic signaling. Cell 96, 615-624.

Czabotar, P.E., Lee, E.F., van Delft, M.F., Day, C.L., Smith, B.J., Huang, D.C., Fairlie, W.D., Hinds, M.G., and Colman, P.M. (2007). Structural insights into the degradation of Mcl-1 induced by BH3 domains. Proc Natl Acad Sci U S A 104, 6217-6222.

Danial, N.N., and Korsmeyer, S.J. (2004). Cell death: critical control points. Cell 116, 205-219. 
Day, C.L., Chen, L., Richardson, S.J., Harrison, P.J., Huang, D.C., and Hinds, M.G. (2005). Solution structure of prosurvival Mcl-1 and characterization of its binding by proapoptotic BH3-only ligands. J Biol Chem 280, 4738-4744.

Day, C.L., Smits, C., Fan, F.C., Lee, E.F., Fairlie, W.D., and Hinds, M.G. (2008).

Structure of the BH3 domains from the p53-inducible BH3-only proteins Noxa and Puma in complex with Mcl-1. J Mol Biol 380, 958-971.

Delaglio, F., Grzesiek, S., Vuister, G.W., Zhu, G., Pfeifer, J., and Bax, A. (1995).

NMRPipe: a multidimensional spectral processing system based on UNIX pipes. J Biomol NMR 6, 277-293.

Denisov, A.Y., Chen, G., Sprules, T., Moldoveanu, T., Beauparlant, P., and Gehring, K. (2006). Structural model of the BCL-w-BID peptide complex and its interactions with phospholipid micelles. Biochemistry 45, 2250-2256.

Denisov, A.Y., Sprules, T., Fraser, J., Kozlov, G., and Gehring, K. (2007a). Heat-Induced Dimerization of BCL-x(L) through alpha-Helix Swapping. Biochemistry 46, 734-740.

Denisov, A.Y., Sprules, T., Fraser, J., Kozlov, G., and Gehring, K. (2007b). Heat-induced dimerization of BCL-xL through alpha-helix swapping. Biochemistry 46, 734-740.

Dijkers, P.F., Medema, R.H., Lammers, J.W., Koenderman, L., and Coffer, P.J. (2000). Expression of the pro-apoptotic Bcl-2 family member Bim is regulated by the forkhead transcription factor FKHR-L1. Curr Biol 10, 1201-1204.

Dombkowski, A.A. (2003). Disulfide by Design: a computational method for the rational design of disulfide bonds in proteins. Bioinformatics 19, 1852-1853.

Dyson, H.J., and Wright, P.E. (2005). Intrinsically unstructured proteins and their functions. Nat Rev Mol Cell Biol 6, 197-208.

Egle, A., Harris, A.W., Bouillet, P., and Cory, S. (2004). Bim is a suppressor of Mycinduced mouse B cell leukemia. Proc Natl Acad Sci U S A 101, 6164-6169.

Eischen, C.M., Weber, J.D., Roussel, M.F., Sherr, C.J., and Cleveland, J.L. (1999a).

Disruption of the ARF-Mdm2-p53 tumor suppressor pathway in Myc-induced lymphomagenesis. Genes Dev 13, 2658-2669.

Eischen, C.M., Weber, J.D., Roussel, M.F., Sherr, C.J., and Cleveland, J.L. (1999b).

Disruption of the ARF-Mdm2-p53 tumor suppressor pathway in Myc-induced lymphomagenesis. Genes Dev 13, 2658-2669.

Emsley, P., and Cowtan, K. (2004). Coot: model-building tools for molecular graphics. Acta Crystallogr D Biol Crystallogr 60, 2126-2132.

Evan, G., and Littlewood, T. (1998). A matter of life and cell death. Science 281, 13171322. 
Fadok, V.A., Bratton, D.L., Rose, D.M., Pearson, A., Ezekewitz, R.A., and Henson, P.M. (2000). A receptor for phosphatidylserine-specific clearance of apoptotic cells. Nature $405,85-90$.

Feng, W., Huang, S., Wu, H., and Zhang, M. (2007). Molecular basis of Bcl-xL's target recognition versatility revealed by the structure of $\mathrm{Bcl}-\mathrm{xL}$ in complex with the $\mathrm{BH} 3$ domain of Beclin-1. J Mol Biol 372, 223-235.

Frisch, S.M., and Screaton, R.A. (2001). Anoikis mechanisms. Curr Opin Cell Biol 13, $555-562$.

Fu, X., Apgar, J.R., and Keating, A.E. (2007). Modeling backbone flexibility to achieve sequence diversity: the design of novel alpha-helical ligands for Bcl-xL. J Mol Biol 371, 1099-1117.

Fuxreiter, M., Tompa, P., and Simon, I. (2007). Local structural disorder imparts plasticity on linear motifs. Bioinformatics 23, 950-956.

Garrison, S.P., Jeffers, J.R., Yang, C., Nilsson, J.A., Hall, M.A., Rehg, J.E., Yue, W., Yu, J., Zhang, L., Onciu, M., et al. (2008). Selection against PUMA gene expression in Mycdriven B-cell lymphomagenesis. Mol Cell Biol 28, 5391-5402.

Gill, S.C., and von Hippel, P.H. (1989). Calculation of protein extinction coefficients from amino acid sequence data. Anal Biochem 182, 319-326.

Green, D.R., and Evan, G.I. (2002). A matter of life and death. Cancer Cell 1, 19-30.

Hajduk, P.J., Meadows, R.P., and Fesik, S.W. (1997). Discovering high-affinity ligands for proteins. Science 278, 497,499.

Hanahan, D., and Weinberg, R.A. (2000). The hallmarks of cancer. Cell 100, 57-70.

Hanayama, R., Tanaka, M., Miwa, K., Shinohara, A., Iwamatsu, A., and Nagata, S. (2002). Identification of a factor that links apoptotic cells to phagocytes. Nature 417, 182187.

Hao, Z., Duncan, G.S., Chang, C.C., Elia, A., Fang, M., Wakeham, A., Okada, H., Calzascia, T., Jang, Y., You-Ten, A., et al. (2005). Specific ablation of the apoptotic functions of cytochrome $\mathrm{C}$ reveals a differential requirement for cytochrome $\mathrm{C}$ and Apaf1 in apoptosis. Cell 121, 579-591.

Harrington, E.A., Fanidi, A., and Evan, G.I. (1994). Oncogenes and cell death. Curr Opin Genet Dev 4, 120-129.

Haupt, Y., Rowan, S., Shaulian, E., Vousden, K.H., and Oren, M. (1995). Induction of apoptosis in HeLa cells by trans-activation-deficient p53. Genes Deve 9, 2180-2183. 
Hemann, M.T., Zilfou, J.T., Zhao, Z., Burgess, D.J., Hannon, G.J., and Lowe, S.W. (2004). Suppression of tumorigenesis by the p53 target PUMA. Proc Natl Acad Sci U S A $101,9333-9338$.

Hengartner, M.O., Ellis, R.E., and Horvitz, H.R. (1992). Caenorhabditis elegans gene ced-9 protects cells from programmed cell death. Nature 356, 494-499.

Herman, M.D., Nyman, T., Welin, M., Lehtio, L., Flodin, S., Tresaugues, L., Kotenyova, T., Flores, A., and Nordlund, P. (2008). Completing the family portrait of the antiapoptotic Bcl-2 proteins: crystal structure of human Bfl-1 in complex with Bim. FEBS Lett 582, 3590-3594.

Hinds, M.G., Smits, C., Fredericks-Short, R., Risk, J.M., Bailey, M., Huang, D.C., and Day, C.L. (2006). Bim, Bad and Bmf: intrinsically unstructured BH3-only proteins that undergo a localized conformational change upon binding to prosurvival Bcl-2 targets. Cell Death Differ 14, 128-136.

Hinds, M.G., Smits, C., Fredericks-Short, R., Risk, J.M., Bailey, M., Huang, D.C., and Day, C.L. (2007). Bim, Bad and Bmf: intrinsically unstructured BH3-only proteins that undergo a localized conformational change upon binding to prosurvival Bcl-2 targets. Cell Death Differ 14, 128-136.

Hwang, T.L.a.S., A.J. (1995). Water suppression using excitation sculpting with gradients. Journal of Magnetic Resonance, Series A 112, 275-279.

Ionov, Y., Yamamoto, H., Krajewski, S., Reed, J.C., and Perucho, M. (2000). Mutational inactivation of the proapoptotic gene BAX confers selective advantage during tumor clonal evolution. Proc Natl Acad Sci U S A 97, 10872-10877.

Jeffers, J.R., Parganas, E., Lee, Y., Yang, C., Wang, J., Brennan, J., MacLean, K.H., Han, J., Chittenden, T., Ihle, J.N., et al. (2003). Puma is an essential mediator of p53dependent and -independent apoptotic pathways. Cancer Cell 4, 321-328.

Jeong, S.Y., Gaume, B., Lee, Y.J., Hsu, Y.T., Ryu, S.W., Yoon, S.H., and Youle, R.J. (2004). Bcl-x(L) sequesters its C-terminal membrane anchor in soluble, cytosolic homodimers. EMBO J 23, 2146-2155.

Jiang, M., Wei, Q., Wang, J., Du, Q., Yu, J., Zhang, L., and Dong, Z. (2006). Regulation of PUMA-alpha by p53 in cisplatin-induced renal cell apoptosis. Oncogene $25,4056-$ 4066 .

Karst, A.M., Dai, D.L., Martinka, M., and Li, G. (2005). PUMA expression is significantly reduced in human cutaneous melanomas. Oncogene 24, 1111-1116.

Kaufmann, T., Tai, L., Ekert, P.G., Huang, D.C., Norris, F., Lindemann, R.K., Johnstone, R.W., Dixit, V.M., and Strasser, A. (2007). The BH3-only protein bid is dispensable for DNA damage- and replicative stress-induced apoptosis or cell-cycle arrest. Cell 129, 423433. 
Kerr, J.F., Wyllie, A.H., and Currie, A.R. (1972). Apoptosis: a basic biological phenomenon with wide-ranging implications in tissue kinetics. Br J Cancer 26, 239-257.

Kluck, R.M., Bossy-Wetzel, E., Green, D.R., and Newmeyer, D.D. (1997). The release of cytochrome $\mathrm{c}$ from mitochondria: a primary site for $\mathrm{Bcl}-2$ regulation of apoptosis. Science 275, 1132-1136.

Kriwacki, R.W., Hengst, L., Tennant, L., Reed, S.I., and Wright, P.E. (1996). Structural studies of $\mathrm{p} 21$ (waf1/cip1/sdi1) in the free and Cdk2-bound state: Conformational disorder mediates binding diversity. Proc Natl Acad Sci USA 93, 11504-11509.

Kruse, J.P., and Gu, W. (2009). Modes of p53 regulation. Cell 137, 609-622.

Kuwana, T., Bouchier-Hayes, L., Chipuk, J.E., Bonzon, C., Sullivan, B.A., Green, D.R., and Newmeyer, D.D. (2005). BH3 domains of BH3-only proteins differentially regulate Bax-mediated mitochondrial membrane permeabilization both directly and indirectly. Mol Cell 17, 525-535.

Kuwana, T., Mackey, M.R., Perkins, G., Ellisman, M.H., Latterich, M., Schneiter, R., Green, D.R., and Newmeyer, D.D. (2002). Bid, Bax, and lipids cooperate to form supramolecular openings in the outer mitochondrial membrane. Cell 111,331-342.

Kvansakul, M., Yang, H., Fairlie, W.D., Czabotar, P.E., Fischer, S.F., Perugini, M.A., Huang, D.C., and Colman, P.M. (2008). Vaccinia virus anti-apoptotic F1L is a novel Bcl2-like domain-swapped dimer that binds a highly selective subset of BH3-containing death ligands. Cell Death Differ 15, 1564-1571.

Labi, V., Erlacher, M., Kiessling, S., and Villunger, A. (2006). BH3-only proteins in cell death initiation, malignant disease and anticancer therapy. Cell Death Differ 13, 13251338.

Lacy, E.R., Filippov, I., Lewis, W.S., Otieno, S., Xiao, L., Weiss, S., Hengst, L., and Kriwacki, R.W. (2004). p27 binds cyclin-CDK complexes through a sequential mechanism involving binding-induced protein folding. Nat Struct Mol Biol 11, 358-364.

Lakowicz, J.R. (1983). Principles of Fluorescence Spectroscopy (New York, Plenum Press).

Lane, D.P. (1992). Cancer. p53, guardian of the genome. Nature 358, 15-16.

Laue, T.M., Shah, B.D., Ridgeway, T.M., and , and Pelletier, S.L. (1992). Analytical Ultracentrifugation in Biochemistry and Polymer Science In, S.E. Harding, A.J. Rowe, and J.C. Horton, eds. (Cambridge, The Royal Society of Chemistry, ), pp. 90-125.

Leber, B., Lin, J., and Andrews, D.W. (2007). Embedded together: the life and death consequences of interaction of the Bcl-2 family with membranes. Apoptosis 12, 897-911. 
Lee, E.F., Czabotar, P.E., Smith, B.J., Deshayes, K., Zobel, K., Colman, P.M., and Fairlie, W.D. (2007). Crystal structure of ABT-737 complexed with Bcl-xL: implications for selectivity of antagonists of the Bcl-2 family. Cell Death Differ 14, 1711-1713.

Lee, E.F., Czabotar, P.E., Yang, H., Sleebs, B.E., Lessene, G., Colman, P.M., Smith, B.J., and Fairlie, W.D. (2009). Conformational changes in Bcl-2 pro-survival proteins determine their capacity to bind ligands. J Biol Chem 284, 30508-30517.

Letai, A., Bassik, M.C., Walensky, L.D., Sorcinelli, M.D., Weiler, S., and Korsmeyer, S.J. (2002). Distinct BH3 domains either sensitize or activate mitochondrial apoptosis, serving as prototype cancer therapeutics. Cancer Cell 2, 183-192.

Letai, A.G. (2008). Diagnosing and exploiting cancer's addiction to blocks in apoptosis. Nat Rev Cancer 8, 121-132.

Leu, J.I., Dumont, P., Hafey, M., Murphy, M.E., and George, D.L. (2004). Mitochondrial p53 activates Bak and causes disruption of a Bak-Mcl1 complex. Nat Cell Biol 6, 443450.

Ley, R., Ewings, K.E., Hadfield, K., and Cook, S.J. (2005). Regulatory phosphorylation of Bim: sorting out the ERK from the JNK. Cell Death Differ 12, 1008-1014.

Li, H., Zhu, H., Xu, C.J., and Yuan, J. (1998). Cleavage of BID by caspase 8 mediates the mitochondrial damage in the Fas pathway of apoptosis. Cell 94, 491-501.

Li, P., Nijhawan, D., Budihardjo, I., Srinivasula, S.M., Ahmad, M., Alnemri, E.S., and Wang, X. (1997). Cytochrome c and dATP-dependent formation of Apaf-1/caspase-9 complex initiates an apoptotic protease cascade. Cell 91, 479-489.

Liu, X., Dai, S., Zhu, Y., Marrack, P., and Kappler, J.W. (2003). The structure of a Bcl$\mathrm{xL} /$ Bim fragment complex: implications for Bim function. Immunity 19, 341-352.

Liu, Y., and Eisenberg, D. (2002). 3D domain swapping: as domains continue to swap. Protein Sci 11, 1285-1299.

Louie, G.V., Yang, W., Bowman, M.E., and Choe, S. (1997). Crystal structure of the complex of diphtheria toxin with an extracellular fragment of its receptor. Mol Cell 1, 6778 .

Lovell, J.F., Billen, L.P., Bindner, S., Shamas-Din, A., Fradin, C., Leber, B., and Andrews, D.W. (2008). Membrane binding by tBid initiates an ordered series of events culminating in membrane permeabilization by Bax. Cell 135, 1074-1084.

Luo, X., Budihardjo, I., Zou, H., Slaughter, C., and Wang, X. (1998). Bid, a Bcl2 interacting protein, mediates cytochrome c release from mitochondria in response to activation of cell surface death receptors. Cell 94, 481-490. 
Manion, M.K., O'Neill, J.W., Giedt, C.D., Kim, K.M., Zhang, K.Y., and Hockenbery, D.M. (2004). Bcl-XL mutations suppress cellular sensitivity to antimycin A. J Biol Chem 279, 2159-2165.

McRee, D.E., and Israel, M. (2008). XtalView, protein structure solution and protein graphics, a short history. J Struct Biol 29, 29.

Merino, D., Giam, M., Hughes, P.D., Siggs, O.M., Heger, K., O'Reilly, L.A., Adams, J.M., Strasser, A., Lee, E.F., Fairlie, W.D., et al. (2009). The role of BH3-only protein Bim extends beyond inhibiting Bcl-2-like prosurvival proteins. J Cell Biol 186, 355-362.

Mihara, M., Erster, S., Zaika, A., Petrenko, O., Chittenden, T., Pancoska, P., and Moll, U.M. (2003). p53 has a direct apoptogenic role at the mitochondria. Mol Cell 11, 577 590 .

Mittag, T., and Forman-Kay, J.D. (2007). Atomic-level characterization of disordered protein ensembles. Curr Opin Struct Biol 17, 3-14.

Mora, J., Cheung, N.K., Chen, L., Qin, J., and Gerald, W. (2001). Loss of heterozygosity at $19 q 13.3$ is associated with locally aggressive neuroblastoma. Clin Cancer Res 7, 13581361.

Muchmore, S.W., Sattler, M., Liang, H., Meadows, R.P., Harlan, J.E., Yoon, H.S., Nettesheim, D., Chang, B.S., Thompson, C.B., Wong, S.L., et al. (1996a). X-ray and NMR structure of human Bcl-xL, an inhibitor of programmed cell death. Nature 381, 335-341.

Muchmore, S.W., Sattler, M., Liang, H., Meadows, R.P., Harlan, J.E., Yoon, H.S., Nettesheim, D., Chang, B.S., Thompson, C.B., Wong, S.L., et al. (1996b). X-ray and NMR structure of human Bcl-xL, an inhibitor of programmed cell death. Nature 381, $335-341$.

Munoz-Pinedo, C., Guio-Carrion, A., Goldstein, J.C., Fitzgerald, P., Newmeyer, D.D., and Green, D.R. (2006). Different mitochondrial intermembrane space proteins are released during apoptosis in a manner that is coordinately initiated but can vary in duration. Proc Natl Acad Sci U S A 103, 11573-11578.

Muppidi, J.R., Tschopp, J., and Siegel, R.M. (2004). Life and death decisions: secondary complexes and lipid rafts in TNF receptor family signal transduction. Immunity 21, 461465.

Murshudov, G.N., Vagin, A.A., and Dodson, E.J. (1997). Refinement of macromolecular structures by the maximum-likelihood method. Acta Crystallogr D Biol Crystallogr 53, 240-255.

Nakano, K., and Vousden, K.H. (2001). PUMA, a novel proapoptotic gene, is induced by p53. Mol Cell 7, 683-694. 
O'Neill, J.W., Manion, M.K., Maguire, B., and Hockenbery, D.M. (2006). BCL-XL dimerization by three-dimensional domain swapping. J Mol Biol 356, 367-381.

Oberst, A., Pop, C., Tremblay, A.G., Blais, V., Denault, J.B., Salvesen, G.S., and Green, D.R. (2010). Inducible dimerization and inducible cleavage reveal a requirement for both processes in caspase- 8 activation. J Biol Chem 285, 16632-16642.

Oda, E., Ohki, R., Murasawa, H., Nemoto, J., Shibue, T., Yamashita, T., Tokino, T., Taniguchi, T., and Tanaka, N. (2000). Noxa, a BH3-only member of the Bcl-2 family and candidate mediator of p53-induced apoptosis. Science 288, 1053-1058.

Oltersdorf, T., Elmore, S.W., Shoemaker, A.R., Armstrong, R.C., Augeri, D.J., Belli, B.A., Bruncko, M., Deckwerth, T.L., Dinges, J., Hajduk, P.J., et al. (2005). An inhibitor of Bcl-2 family proteins induces regression of solid tumours. Nature 435, 677-681.

Otwinowski, Z., and Minor, W. (1997). Processing of X-ray Diffraction Data Collected in Oscillation Mode. Meth Enzymol 276, 307-326.

Petros, A.M., Gunasekera, A., Xu, N., Olejniczak, E.T., and Fesik, S.W. (2004a). Defining the p53 DNA-binding domain/Bcl-x(L)-binding interface using NMR. FEBS Lett 559, 171-174.

Petros, A.M., Nettesheim, D.G., Wang, Y., Olejniczak, E.T., Meadows, R.P., Mack, J., Swift, K., Matayoshi, E.D., Zhang, H., Thompson, C.B., et al. (2000). Rationale for Bcl$\mathrm{xL} / \mathrm{Bad}$ peptide complex formation from structure, mutagenesis, and biophysical studies. Protein Sci 9, 2528-2534.

Petros, A.M., Olejniczak, E.T., and Fesik, S.W. (2004b). Structural biology of the Bcl-2 family of proteins. Biochim Biophys Acta 1644, 83-94.

Pham, T.C., Kriwacki, R.W., and Parrill, A.L. (2007). Peptide design and structural characterization of a GPCR loop mimetic. Biopolymers 86, 298-310.

Pop, C., Timmer, J., Sperandio, S., and Salvesen, G.S. (2006). The apoptosome activates caspase- 9 by dimerization. Mol Cell 22, 269-275.

Puthalakath, H., Huang, D.C., O'Reilly, L.A., King, S.M., and Strasser, A. (1999). The proapoptotic activity of the Bcl-2 family member Bim is regulated by interaction with the dynein motor complex. Mol Cell 3, 287-296.

Puthalakath, H., O'Reilly, L.A., Gunn, P., Lee, L., Kelly, P.N., Huntington, N.D., Hughes, P.D., Michalak, E.M., McKimm-Breschkin, J., Motoyama, N., et al. (2007). ER stress triggers apoptosis by activating BH3-only protein Bim. Cell 129, 1337-1349.

Puthalakath, H., Villunger, A., O'Reilly, L.A., Beaumont, J.G., Coultas, L., Cheney, R.E., Huang, D.C., and Strasser, A. (2001). Bmf: a proapoptotic BH3-only protein regulated by interaction with the myosin V actin motor complex, activated by anoikis. Science 293, 1829-1832. 
Reed, J.C. (1997). Bcl-2 family proteins: regulators of apoptosis and chemoresistance in hematologic malignancies. Semin Hematol 34, 9-19.

Reed, J.C. (1999). Dysregulation of apoptosis in cancer. J Clin Oncol 17, 2941-2953.

Riedl, S.J., and Salvesen, G.S. (2007). The apoptosome: signalling platform of cell death. Nat Rev Mol Cell Biol 8, 405-413.

Rubin, B.Y., Smith, L.J., Hellermann, G.R., Lunn, R.M., Richardson, N.K., and Anderson, S.L. (1988). Correlation between the anticellular and DNA fragmenting activities of tumor necrosis factor. Cancer Res 48, 6006-6010.

Salvesen, G.S., and Dixit, V.M. (1997). Caspases: intracellular signaling by proteolysis. Cell 91, 443-446.

Sattler, M., Liang, H., Nettesheim, D., Meadows, R.P., Harlan, J.E., Eberstadt, M., Yoon, H.S., Shuker, S.B., Chang, B.S., Minn, A.J., et al. (1997). Structure of Bcl-xL-Bak peptide complex: recognition between regulators of apoptosis. Science 275, 983-986.

Savill, J., and Fadok, V. (2000). Corpse clearance defines the meaning of cell death. Nature 407, 784-788.

Schleucher, J., Schwendinger, M., Sattler, M., Schmidt, P., Schedletzky, O., Glaser, S.J., Sorensen, O.W., and Griesinger, C. (1994). A general enhancement scheme in heteronuclear multidimensional NMR employing pulsed field gradients. J Biomol NMR 4, 301-306.

Schuck, P. (2000). Size-distribution analysis of macromolecules by sedimentation velocity ultracentrifugation and lamm equation modeling. Biophys J 78, 1606-1619.

Schuck, P., Perugini, M.A., Gonzales, N.R., Howlett, G.J., and Schubert, D. (2002). Sizedistribution analysis of proteins by analytical ultracentrifugation: strategies and application to model systems. Biophys J 82, 1096-1111.

Shimazaki, C., Inaba, T., and Nakagawa, M. (2000). B-cell lymphoma-associated hemophagocytic syndrome. Leuk Lymphoma 38, 121-130.

Shimazu, T., Degenhardt, K., Nur, E.K.A., Zhang, J., Yoshida, T., Zhang, Y., Mathew, R., White, E., and Inouye, M. (2007). NBK/BIK antagonizes MCL-1 and BCL-XL and activates BAK-mediated apoptosis in response to protein synthesis inhibition. Genes Dev 21, 929-941.

Sivakolundu, S.G., Bashford, D., and Kriwacki, R.W. (2005). Disordered p27Kip1 exhibits intrinsic structure resembling the Cdk2/cyclin A-bound conformation. J Mol Biol $353,1118-1128$.

Smits, C., Czabotar, P.E., Hinds, M.G., and Day, C.L. (2008). Structural plasticity underpins promiscuous binding of the prosurvival protein A1. Structure 16, 818-829. 
Sot, B., Freund, S.M., and Fersht, A.R. (2007). Comparative Biophysical Characterization of p53 with the Pro-apoptotic BAK and the Anti-apoptotic BCL-xL. J Biol Chem 282, 29193-29200.

Speidel, D., Helmbold, H., and Deppert, W. (2006). Dissection of transcriptional and non-transcriptional p53 activities in the response to genotoxic stress. Oncogene 25, 940953.

Suzuki, M., Youle, R.J., and Tjandra, N. (2000). Structure of Bax: coregulation of dimer formation and intracellular localization. Cell 103, 645-654.

Tait, S.W., and Green, D.R. (2010). Mitochondria and cell death: outer membrane permeabilization and beyond. Nat Rev Mol Cell Biol 11, 621-632.

Tomita, Y., Marchenko, N., Erster, S., Nemajerova, A., Dehner, A., Klein, C., Pan, H., Kessler, H., Pancoska, P., and Moll, U.M. (2006). WT p53, but not tumor-derived mutants, bind to $\mathrm{Bcl} 2$ via the DNA binding domain and induce mitochondrial permeabilization. J Biol Chem 281, 8600-8606. Epub 2006 Jan 8626.

Toth, A., Jeffers, J.R., Nickson, P., Min, J.Y., Morgan, J.P., Zambetti, G.P., and Erhardt, P. (2006). Targeted deletion of Puma attenuates cardiomyocyte death and improves cardiac function during ischemia-reperfusion. Am J Physiol Heart Circ Physiol 291, H5260 .

Uren, R.T., Dewson, G., Chen, L., Coyne, S.C., Huang, D.C., Adams, J.M., and Kluck, R.M. (2007). Mitochondrial permeabilization relies on $\mathrm{BH} 3$ ligands engaging multiple prosurvival Bcl-2 relatives, not Bak. J Cell Biol 177, 277-287.

Vacic, V., Oldfield, C.J., Mohan, A., Radivojac, P., Cortese, M.S., Uversky, V.N., and Dunker, A.K. (2007). Characterization of molecular recognition features, MoRFs, and their binding partners. J Proteome Res 6, 2351-2366.

van Delft, M.F., Wei, A.H., Mason, K.D., Vandenberg, C.J., Chen, L., Czabotar, P.E., Willis, S.N., Scott, C.L., Day, C.L., Cory, S., et al. (2006). The BH3 mimetic ABT-737 targets selective Bcl-2 proteins and efficiently induces apoptosis via Bak/Bax if $\mathrm{Mcl}-1$ is neutralized. Cancer Cell 10, 389-399.

Villunger, A., Michalak, E.M., Coultas, L., Mullauer, F., Bock, G., Ausserlechner, M.J., Adams, J.M., and Strasser, A. (2003). p53- and drug-induced apoptotic responses mediated by BH3-only proteins puma and noxa. Science 302, 1036-1038. Epub 2003 Sep 1018.

Vogelstein, B., Lane, D., and Levine, A.J. (2000). Surfing the p53 network. Nature 408, 307-310.

Vousden, K.H., and Prives, C. (2009). Blinded by the Light: The Growing Complexity of p53. Cell 137, 413-431. 
Wang, Y., Fisher, J.C., Mathew, R., Ou, L., Otieno, S., Sublet, J., Xiao, L., Chen, J., Roussel, M.F., and Kriwacki, R.W. (2011). Intrinsic disorder mediates the diverse regulatory functions of the Cdk inhibitor p21. Nat Chem Biol 7, 214-221.

Wei, M.C. (2004). Bcl-2-related genes in lymphoid neoplasia. Int J Hematol 80, 205-209.

Wei, M.C., Lindsten, T., Mootha, V.K., Weiler, S., Gross, A., Ashiya, M., Thompson, C.B., and Korsmeyer, S.J. (2000). tBID, a membrane-targeted death ligand, oligomerizes BAK to release cytochrome c. Genes Dev 14, 2060-2071.

Wei, M.C., Zong, W.X., Cheng, E.H., Lindsten, T., Panoutsakopoulou, V., Ross, A.J., Roth, K.A., MacGregor, G.R., Thompson, C.B., and Korsmeyer, S.J. (2001).

Proapoptotic BAX and BAK: a requisite gateway to mitochondrial dysfunction and death. Science 292, 727-730.

Willis, S.N., Fletcher, J.I., Kaufmann, T., van Delft, M.F., Chen, L., Czabotar, P.E., Ierino, H., Lee, E.F., Fairlie, W.D., Bouillet, P., et al. (2007). Apoptosis initiated when BH3 ligands engage multiple Bcl-2 homologs, not Bax or Bak. Science 315, 856-859.

Yang, E., Zha, J., Jockel, J., Boise, L.H., Thompson, C.B., and Korsmeyer, S.J. (1995). $\mathrm{Bad}$, a heterodimeric partner for Bcl-XL and Bcl-2, displaced Bax and promotes cell death. Cell 80, 285-291.

Yang, J., Liu, X., Bhalla, K., Kim, C.N., Ibrado, A.M., Cai, J., Peng, T.I., Jones, D.P., and Wang, X. (1997). Prevention of apoptosis by Bcl-2: release of cytochrome c from mitochondria blocked. Science 275, 1129-1132.

Yin, X.M., Wang, K., Gross, A., Zhao, Y., Zinkel, S., Klocke, B., Roth, K.A., and Korsmeyer, S.J. (1999). Bid-deficient mice are resistant to Fas-induced hepatocellular apoptosis. Nature 400, 886-891.

Yong, W.H., Ueki, K., Chou, D., Reeves, S.A., von Deimling, A., Gusella, J.F., Mohrenweiser, H.W., Buckler, A.J., and Louis, D.N. (1995). Cloning of a highly conserved human protein serine-threonine phosphatase gene from the glioma candidate region on chromosome 19q13.3. Genomics 29, 533-536.

Youle, R.J., and Strasser, A. (2008). The BCL-2 protein family: opposing activities that mediate cell death. Nat Rev Mol Cell Biol 9, 47-59.

Yu, J., Zhang, L., Hwang, P.M., Kinzler, K.W., and Vogelstein, B. (2001). PUMA induces the rapid apoptosis of colorectal cancer cells. Mol Cell 7, 673-682.

Zha, J., Harada, H., Yang, E., Jockel, J., and Korsmeyer, S.J. (1996). Serine phosphorylation of death agonist BAD in response to survival factor results in binding to 14-3-3 not BCL-X(L). Cell 87, 619-628. 
Zhu, G., Kong, X.M., and Sze, K.H. (1999). Gradient and sensitivity enhancement of 2D TROSY with water flip-back, 3D NOESY-TROSY and TOCSY-TROSY experiments. J Biomol NMR 13, 77-81.

Zou, H., Henzel, W.J., Liu, X., Lutschg, A., and Wang, X. (1997). Apaf-1, a human protein homologous to C. elegans CED-4, participates in cytochrome c-dependent activation of caspase-3. Cell 90, 405-413.

Zwart, P.H., Afonine, P.V., Grosse-Kunstleve, R.W., Hung, L.W., Ioerger, T.R., McCoy, A.J., McKee, E., Moriarty, N.W., Read, R.J., Sacchettini, J.C., et al. (2008). Automated structure solution with the PHENIX suite. Methods Mol Biol 426, 419-435. 
APPENDIX A. SUPPLIMENTARY TABLES AND FIGURES 
Table A.1 Oligonucleotide Primers for Site-directed Mutagenesis.

\begin{tabular}{|c|c|}
\hline Protein & Oligonucleotide \\
\hline \multirow[b]{2}{*}{ BCL-xL $\Delta C$ E7C } & 5' ATATGTCTCAGAGCAACCGGTGCCTGGTGGTTGACTTTCTCTC 3' \\
\hline & 5' TATACAGAGTCTCGTTGGCCACGGACCACCAACTGAAAGAGAG 3' \\
\hline \multirow{2}{*}{ BCL-xL $\Delta \mathrm{C} H 113 \mathrm{~A}$} & 5' CTGACATCCCAGCTCGCCATCACCCCAGGGAC 3' \\
\hline & 5' GACTGTAGGGTCGAGCGGTAGTGGGGTCCCTG 3' \\
\hline \multirow{2}{*}{ BCL-xL $\Delta C$ V135C } & 5' CCGGGATGGGTGCAACTGGGGTCGCATTGTGGC 3' \\
\hline & 5' GGCCCTACCCACGTTGACCCCAGCGTAACACCG 3' \\
\hline \multirow{2}{*}{ BCL-xL $\Delta C \mathrm{~N} 175 \mathrm{C}$} & 5' GGATGGCCACTTACCTGTGTGACCACCTAGAGCCTT 3' \\
\hline & 5' CCTACCGGTGAATGGACACACTGGTGGATCTCGGAA 3' \\
\hline \multirow{2}{*}{$\mathrm{BCL}-\mathrm{xL} \Delta \mathrm{C} \mathrm{W} 181 \mathrm{C}$} & 5' GACCACCTAGAGCCTTGCATCCAGGAGAACGGC 3' \\
\hline & 5' CTGGTGGATCTCGGAACGTAGGTCCTCTTGCCG 3' \\
\hline \multirow{2}{*}{ PUMA W71A } & 5' CGCGGGGAGGAGGAACAGGCGGCCCGGGAGATC 3' \\
\hline & 5' GATCTCCCGGGCCGCCTGTTCCTCCTCCCCGCG 3' \\
\hline
\end{tabular}


Table A.2 Details of Recombinant Protein Expression Vectors.

\begin{tabular}{ccccc}
\hline Vector Name & Antibiotic Resistance & Fusion Tag & Cleavage Method & Origin \\
\hline BAX-pTB1 & Amp & Intein (N) & 50 mM DTT & Green Lab \\
BCL-xL $\Delta$ C-pET28 & Kan & ${ }^{6}$ His $(\mathrm{N})$ & Thrombin & C.G. Park \\
BCL-xL $\Delta$ L $\Delta$ C-pET21 & Amp & ${ }^{6}$ His (C) & None & Wagner Lab \\
BCL-xL $\Delta$ C E7C-pET28 & Kan & ${ }^{6}$ His (N) & Thrombin & J. Fisher \\
BCL-xL $\Delta$ C H113A-pET28 & Kan & ${ }^{6}$ His (N) & Thrombin & J. Fisher \\
BCL-xL $\Delta$ C N175C-pET28 & Kan & ${ }^{6}$ His (N) & Thrombin & J. Fisher \\
BCL-xL $\Delta$ C V135C,W181C-pET28 & Kan & ${ }^{6}$ His (N) & Thrombin & J. Fisher \\
PUMA beta-pET30 & Kan & ${ }^{6}$ His (N) & Thrombin & Eldering Lab \\
PUMA beta W71A-pET30 & Kan & ${ }^{6}$ His (N) & Thrombin & J. Fisher \\
PUMA beta ${ }^{\mathrm{N}}$-pET30 & Kan & ${ }^{6}$ His (N) & Thrombin & C.G. Park \\
PUMA beta ${ }^{\mathrm{C}}$-pET30 & Kan & ${ }^{6}$ His (N) & Thrombin & C.G. Park \\
\hline
\end{tabular}




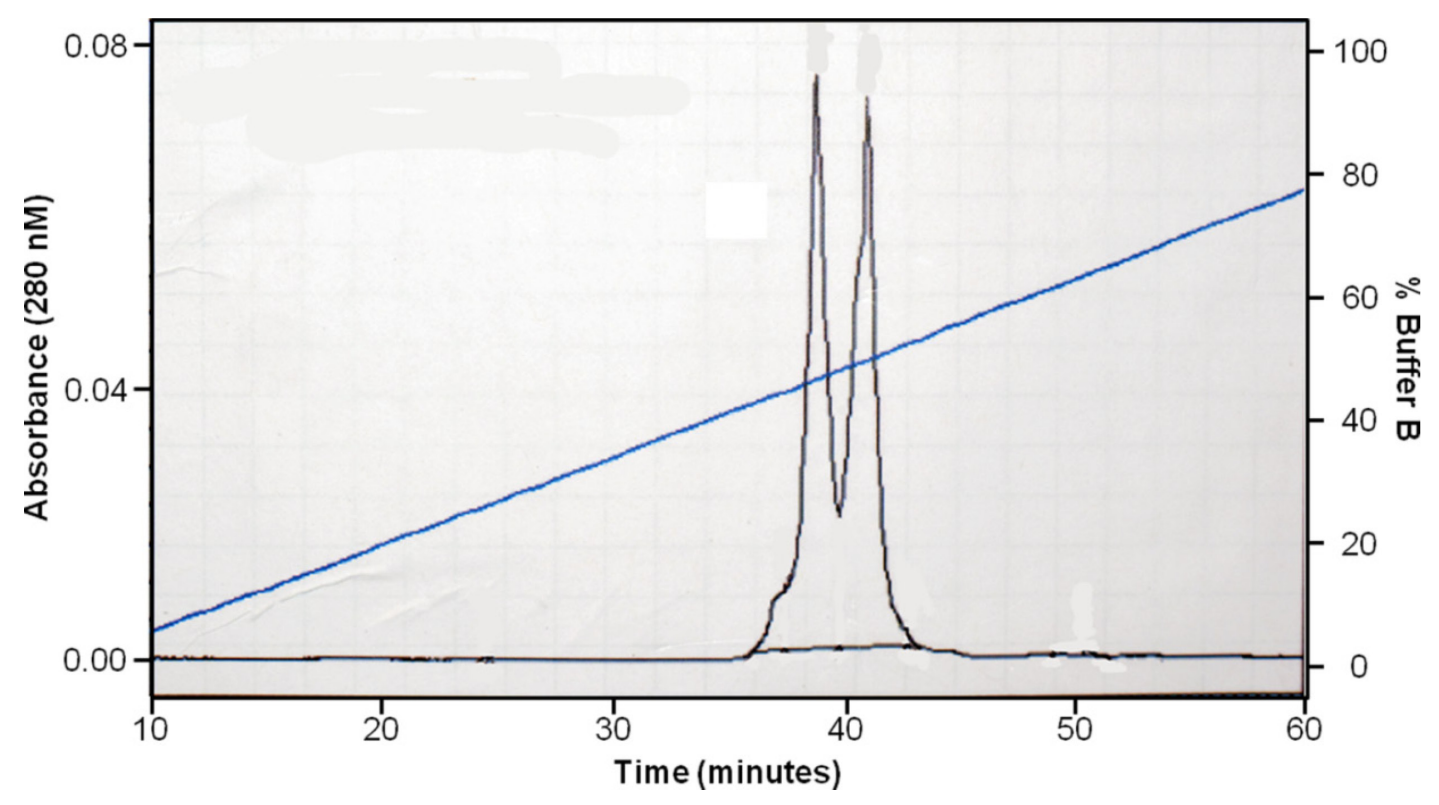

Figure A.1 Reversed Phase C4 HPLC Chromatogram of PUMA.

Shown are the two primary peaks seen during HPLC purification of PUMA. The first peak is discarded and the second peak containing active PUJMA protein is subsequently flash frozen and lyophilized. The blue line indicates the linear acetonitrile gradient used over the course of one hour. The linear acetonitrile gradient was set to increase $1 \%$ each minute for a total of one hour. 
Table A.3 Example of Raw Fluorescence Data Used to Solve for $K_{d}$ by Nonlinear Regression.

\begin{tabular}{cccc}
\hline [BCL-xL] (M) & \% Bound & \% Bound & \% Bound \\
\hline 0.00 & $1.174 \mathrm{e}-006$ & 0.000000 & 0.000000 \\
$2.460000 \mathrm{e}-011$ & 0.0007622 & 0.003850402 & 0.01752863 \\
$4.910000 \mathrm{e}-011$ & 0.0066133 & 0.01733965 & 0.0292946 \\
$2.510000 \mathrm{e}-009$ & 0.2314529 & 0.183359 & 0.2717325 \\
$4.960000 \mathrm{e}-009$ & 0.4408391 & 0.3601685 & 0.4247327 \\
$7.420000 \mathrm{e}-009$ & 0.5570283 & 0.5194997 & 0.5024741 \\
$9.880000 \mathrm{e}-009$ & 0.6227446 & 0.6063511 & 0.5401847 \\
$1.230000 \mathrm{e}-008$ & 0.6573826 & 0.6383788 & 0.5716024 \\
$1.480000 \mathrm{e}-008$ & 0.6843936 & 0.7007385 & 0.594900 \\
$1.720000 \mathrm{e}-008$ & 0.7110994 & 0.7194165 & 0.6251428 \\
$4.180000 \mathrm{e}-008$ & 0.8624737 & 0.8874713 & 0.7938736 \\
$6.640000 \mathrm{e}-008$ & 0.9085243 & 0.9249331 & 0.9243155 \\
$1.890000 \mathrm{e}-007$ & 1.000000 & 1.000000 & 1.000000 \\
\hline
\end{tabular}




\section{WT peptide}

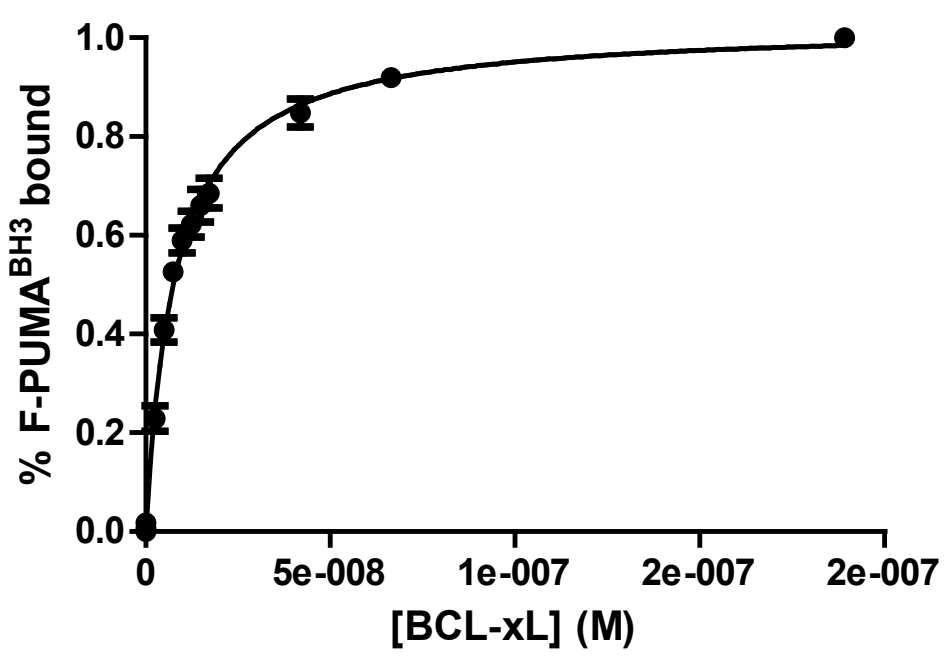

\begin{tabular}{|c|l|}
\hline One site -- Specific binding & \\
\hline Best-fit values & \\
\hline Bmax & 1.025 \\
\hline Kd & $7.798 \mathrm{e}-009$ \\
\hline Std. Error & \\
\hline Bmax & 0.01559 \\
\hline Kd & $3.990 \mathrm{e}-010$ \\
\hline 95\% Confidence Intervals & \\
\hline Bmax & 0.9935 to 1.057 \\
\hline Kd & $6.989 \mathrm{e}-009$ to $8.607 \mathrm{e}-009$ \\
\hline Goodness of Fit & \\
\hline Degrees of Freedom & 37 \\
\hline R square & 0.9902 \\
\hline Absolute Sum of Squares & 0.04239 \\
\hline Sy.x & 0.03385 \\
\hline Number of points & \\
\hline Analyzed & 39 \\
\hline
\end{tabular}

Figure A.2 Determining the $K_{d}$ and $-\Delta \Delta G$ Value for Fluorescein-labeled Wild Type PUMA $^{\text {BH3 }}$.

Non-linear regression plot used to determine the $K_{\mathrm{d}}$ for wild type $\mathrm{PUMA}^{\mathrm{BH} 3}$ (upper panel). The results and corresponding fitting statistics for a set of triplicate data obtained for wild type PUMA ${ }^{\mathrm{BH} 3}$ (lower panel). $\Delta \mathrm{G}$ was determined using the equation: $\Delta \mathrm{G}=-(\mathrm{R})(\mathrm{T}) \ln \left[\left(1 / K_{\mathrm{d}}\right)\right]$. 
Table A.4 Thermodynamic Parameters for BCL-xL Binding to Various BH3 Domain Peptides.

\begin{tabular}{|c|c|c|c|c|}
\hline Interaction & $K_{\mathrm{d}}(\mathrm{nM})$ & $\Delta \mathrm{G}^{\mathrm{a}}\left(\mathrm{kcal}^{\prime} \cdot \mathrm{mol}^{-1}\right)$ & 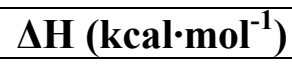 & -T $\Delta S\left(\mathrm{kcal}^{\prime} \mathrm{mol}^{-1}\right)$ \\
\hline $\mathrm{BCL}-\mathrm{xL} \Delta \mathrm{C} \cdot \mathrm{BAD}^{\mathrm{BH} 3}$ & $0.2 \pm 0.3$ & -13.2 & -13.8 & -0.53 \\
\hline $\mathrm{BCL}-\mathrm{xL} \Delta \mathrm{C} \cdot \mathrm{BAK}^{\mathrm{BH} 3}$ & $27.5 \pm 30$ & -10.31 & -7.96 & 2.35 \\
\hline $\mathrm{BCL}-\mathrm{xL} \Delta \mathrm{C} \cdot \mathrm{BAX}^{\mathrm{BH} 3}$ & $111.90 \pm 75$ & -9.47 & -8.88 & 0.60 \\
\hline $\mathrm{BCL}-\mathrm{xL} \Delta \mathrm{C} \cdot \mathrm{BID}^{\mathrm{BH} 3}$ & $10.1 \pm 15$ & -10.9 & -8.16 & 2.74 \\
\hline $\mathrm{BCL}-\mathrm{xL} \Delta \mathrm{C} \cdot \mathrm{BIM}^{\mathrm{BH} 3}$ & $0.50 \pm 6.6$ & -12.7 & -10.6 & 2.07 \\
\hline BCL-xL $\Delta C \mathrm{HRK}^{\mathrm{BH} 3}$ & $28.8 \pm 35$ & -10.3 & -13.0 & -2.70 \\
\hline BCL-xL $\Delta C \cdot$ Noxa $^{\text {BH3 }}$ & ND & ND & ND & ND \\
\hline BCL-xL $\Delta C \cdot P U M A^{B H} 3$ & $2.93 \pm 1.7$ & -23.9 & -16.34 & 7.57 \\
\hline
\end{tabular}

Notes: In all of the above experiment, the binding stoichiometry was determined by fitting the data to a 1:1 binding model. Errors represent the standard deviations from the mean of at least two independent experiments.

${ }^{a}$ Calculated using the equation $\Delta \mathrm{G}=-\mathrm{RT} \ln \left(K_{\mathrm{a}}\right)$.

${ }^{\mathrm{b}}$ Calculated using the equation $\Delta \mathrm{G}=\Delta \mathrm{H}-\mathrm{t} \Delta \mathrm{S}$. 
APPENDIX B. RECOMBINANT PROTEIN SEQUENCES 


\section{BCL-xL $\Delta C$}

GSHMSQSNRELVVDFLSYKLSQKGYSWSQFSDVEENRTEAPEGTESEMETPSAIN GNPSWHLADSPAVNGATAHSSSLDAREVIPMAAVKQALREAGDEFELRYRRAFS DLTSQLHITPGTAYQSFEQVVNELFRDGVNWGRIVAFFSFGGALCVESVDKEMQ VLVSRIAAWMATYLNDHLEPWIQENGGWDTFVELYGNNAAAESRKGQER

\section{BCL-xL $\Delta \mathbf{L} \Delta \mathbf{C}$}

MSMAMSQSNRELVVDFLSYKLSQKGYSWSQFSDVEENRTEAPEGTESEMETPSA INGNPSWHLADSPAVNGATAHSSSLDAREVIPMAAVKQALREAGDEFELRYRRA FSDLTSQLHITPGTAYQSFEQVVNELFRDGVNWGRIVAFFSFGGALCVESVDKEM QVLVSRIAAWMATYLNDHLEPWIQENGGWDTFVELYGNNAAAESRKGQERLE HHHHHH

\section{BCL-xL $\Delta C$ E7C}

GSHMSQSNRCLVVDFLSYKLSQKGYSWSQFSDVEENRTEAPEGTESEMETPSAI NGNPSWHLADSPAVNGATAHSSSLDAREVIPMAAVKQALREAGDEFELRYRRA FSDLTSQLHITPGTAYQSFEQVVNELFRDGVNWGRIVAFFSFGGALCVESVDKEM QVLVSRIAAWMATYLNDHLEPWIQENGGWDTFVELYGNNAAAESRKGQER

\section{BCL-xL $\Delta C$ N175C}

GSHMSQSNRELVVDFLSYKLSQKGYSWSQFSDVEENRTEAPEGTESEMETPSAIN GNPSWHLADSPAVNGATAHSSSLDAREVIPMAAVKQALREAGDEFELRYRRAFS DLTSQLHITPGTAYQSFEQVVNELFRDGVNWGRIVAFFSFGGALCVESVDKEMQ VLVSRIAAWMATYLCDHLEPWIQENGGWDTFVELYGNNAAAESRKGQER

\section{BCL-xL $\Delta C$ V135C,W181C}

GSHMSQSNRELVVDFLSYKLSQKGYSWSQFSDVEENRTEAPEGTESEMETPSAIN GNPSWHLADSPAVNGATAHSSSLDAREVIPMAAVKQALREAGDEFELRYRRAFS DLTSQLHITPGTAYQSFEQVVNELFRDGCNWGRIVAFFSFGGALCVESVDKEMQ VLVSRIAAWMATYLNDHLEPCIQENGGWDTFVELYGNNAAAESRKGQER

\section{GST-BFL-1 $\Delta C$}

GPPEFTMTDCEFGYIYRLAQDYLQCVLQIPQPGSGPSKTSRVLQNVAFSVQKEVE KNLKSCLDNVNVVSVDTARTLFNQVMEKEFEDGIINWGRIVTIFAFEGILIKKLLR QQIAPDVDTYKEISYFVAEFIMNNTGEWIRQNGGWENGFVKKF

\section{MCL-1 $\Delta N \Delta C$}

MHHHHHHEDDLYRQSLEIISRYLREQATGAKDTKPMGRSGATSRKALETLRRVG DGVQRNHETAFQGMLRKLDIKNEDDVKSLSRVMIHVFSDGVTNWGRIVTLISFG 
AFVAKHLKTINQESCIEPLAESITDVLVRTKRDWLVKQRGWDGFVEFFHVEDLE GGIRNVLLAFAGVAGVGAGLAYLIR

\section{PUMA beta}

MHHHHHHSSGLVPRGSGMKETAAAKFERQHMDSPDLGTDDDDKAMK FGMGSAQACPCQVPRAASTTWVPCQICGPQPSLSLAEQHLESPVPSAP GALAGGPTQAAPGVRGEEEQWAREIGAQLRRMADDLNAQYERRRQEE QQRHRPSPWRVLYNLIMGLLPLPRGHRAPEMEPN

PUMA beta ${ }^{\text {W71 } \rightarrow \text { A }}$

MHHHHHHSSGLVPRGSGMKETAAAKFERQHMDSPDLGTDDDDKAMKFGMGS AQACPCQVPRAASTTWVPCQICGPQPSLSLAEQHLESPVPSAPGALAGGPTQAAP GVRGEEEQAAREIGAQLRRMADDLNAQYERRRQEEQQRHRPSPWRVLYNLIMG LLPLPRGHRAPEMEPN

\section{PUMA $^{\mathrm{C}}$}

MHHHHHHSSGLVPRGSGMKETAAAKFERQHMDSPDLGTDDDDKAMAK FGMGSAQACPCQVPRAASTTWVPCQICGPQPSLSLAEQHLESPVPSAP GALAGGPTQAAPGVRGEEEQWAREIGAQLRRMADDLNAQYERRRQEE QQRHRPSPWRVLYNLIMGLLPLPRGHRAPEMEPN

\section{PUMA $^{\mathbf{N}}$}

MHHHHHHSSGLVPRGSGMKETAAAKFERQHMDSPDLGTDDDDKAMK FGMGSAQACPCQVPRAASTTWVPCQICGPQPSLSLAEQHLESPVPSAP GALAGGPTQAAPGVRGE 
APPENDIX C. BUFFERS 


\section{Nickel Affinity Binding Buffer (Lysis Buffer)}

$20 \mathrm{mM}$ Tris, $\mathrm{pH} 8.0$

$5 \mathrm{mM}$ imidazole

$500 \mathrm{mM} \mathrm{NaCl}$

Two EDTA-free protease inhibitor tablets (Roche) per $100 \mathrm{ml}$ buffer

\section{Nickel Elution Buffer}

$20 \mathrm{mM}$ Tris, $\mathrm{pH} 8.0$

$500 \mathrm{mM}$ imidazole

$500 \mathrm{mM} \mathrm{NaCl}$

GST Binding Buffer (Lysis Buffer)

$140 \mathrm{mM} \mathrm{NaCl}$

$2.7 \mathrm{mM} \mathrm{KCl}$

$10 \mathrm{mM} \mathrm{Na}_{2} \mathrm{HPO}_{4}$

$1.8 \mathrm{mM} \mathrm{KH}_{2} \mathrm{PO}_{4}$

Two protease inhibitor tablets (Roche) per $100 \mathrm{ml}$ buffer

GST Elution Buffer

$50 \mathrm{mM}$ Tris, $\mathrm{pH} 8.0$

$10 \mathrm{mM}$ reduced glutathione

Anion Exchange Binding Buffer

$50 \mathrm{mM}$ Tris, $\mathrm{pH} 80=.0$

$10 \mathrm{mM} \mathrm{NaCl}$

Anion Exchange Elution Buffer

$50 \mathrm{mM}$ Tris, $\mathrm{pH} 8.0$

$1.0 \mathrm{M} \mathrm{NaCl}$

Gel Filtration Buffer

50 mM Tris, $\mathrm{pH} 8.0$

$300 \mathrm{mM} \mathrm{NaCl}$

PUMA NMR Buffer

$20 \mathrm{mM}$ deuterated Tris, $\mathrm{pH} 7.1$

$150 \mathrm{mM} \mathrm{NaCl}$

$5 \mathrm{mM}$ deuterated DTT

BCL-xL NMR Buffer

$20 \mathrm{mM}$ sodium phosphate, $\mathrm{pH} 7.0$

$3.0 \mathrm{mM}$ DTT

$0.5 \mathrm{mM}$ EDTA

CD Buffer

$20 \mathrm{mM}$ potassium phosphate, $\mathrm{pH} 7.0$ 
HPLC Column Binding Buffer

$0.1 \% \mathrm{TFA}(\mathrm{V} / \mathrm{V})$ in $\mathrm{H}_{2} \mathrm{O}$

HPLC Column Elution Buffer

$0.1 \% \mathrm{TFA}(\mathrm{V} / \mathrm{V})$ in acetonitrile

Natve PAGE Running Buffer

25 mM Tris, $\mathrm{pH} 8.3$

$192 \mathrm{mM}$ glycine 


\section{VITA}

John C. Fisher was born in Memphis, Tennessee in 1980. He graduated high school from Memphis University School in May of 1997. The following August, he enrolled at The University of Tennessee Knoxville where he completed majors in Biochemistry and Molecular/Cell Biology and French in 2002. He enrolled the University of Tennessee Health Science Center in 2002 as an M.D./Ph.D. student. He is expected to obtain his Ph.D. degree in May 2011.

\section{PUBLICATIONS}

Chipuk, J.E., Fisher, J.C., Dillon, C.P., Kriwacki, R.W., Kuwana, T., and Green, D.R. (2008). Mechanism of apoptosis induction by inhibition of the anti-apoptotic BCL-2 proteins. Proc Natl Acad Sci U S A 105, 20327-20332.

Wang, Y., Fisher, J.C., Mathew, R., Ou, L., Otieno, S., Sublet, J., Xiao, L., Chen, J., Roussel, M.F., and Kriwacki, R.W. (2011). Intrinsic disorder mediates the diverse regulatory functions of the Cdk inhibitor p21. Nat Chem Biol 7, 214-221.

Fisher, J.C., Chipuk'J.E., Mi-Kyung Yun, Nourse,A., Grace,C.R., Baran, K., Min, L., White, S.W., Green,D.R., and Kriwacki, R.W. (in resubmission). The BH3-only protein PUMA induces BCL-xL domain-swapped dimerization that mediates apoptotic signaling. Nature. 